\title{
MAGNETIC INTERACTIONS IN THE SPIN-ORBIT COUPLED ELECTRON GAS
}

A Dissertation presented to

the Faculty of the Graduate School

at the University of Missouri

In Partial Fulfillment

of the Requirements for the Degree

Doctor of Philosophy

by

MOHAMMAD M. VALIZADEH

Prof. Sashi Satpathy, Dissertation Supervisor

December, 2017 
(c) Copyright by Mohammad M. Valizadeh 2017

All Rights Reserved 
The undersigned, appointed by the Dean of the Graduate School, have examined the dissertation entitled:

\section{MAGNETIC INTERACTIONS IN THE SPIN-ORBIT COUPLED ELECTRON GAS}

presented by Mohammad M. Valizadeh, a candidate for the degree of Doctor of Philosophy and hereby certify that, in their opinion, it is worthy of acceptance.

Prof. Sashi Satpathy

Prof. Giovanni Vignale

Prof. Carsten Ullrich

Prof. Guilherme DeSouza 


\section{ACKNOWLEDGMENTS}

I would like to deeply thank my advisor, Prof. Sashi Satpathy, for his support and guidance. I chose University of Missouri because of him and truly appreciate Sashi's trust in my abilities and his great patience with me. During the last five years, he was always available and tried his best to help me. He is a very modest gentleman who treated his students like his colleagues.

I would like to thank Zoran Popovic, Mohammad Sherafati and Eugene Kogan for helpful discussions.

I also would like to thank Prof. Carsten Ullrich, the best Dirctor of Graduate Studies a department can have, and entire faculty, staff and my fellow graduate students in the Department of Physics and Astronomy for providing me with their friendship and support and the Mizzou community. It was a great experience to study at the University of Missouri and is an honor for me to be a member of Mizzou family.

And of course Shahrzad, I want to thank you from the bottom of my heart. During last eight years, you always stood right beside me, and it was not possible for me to reach to this point without your support. I can not thank you enough. 


\section{TABLE OF CONTENTS}

ACKNOWLEDGMENTS ................ ii

LIST OF FIGURES $\ldots \ldots \ldots \ldots \ldots \ldots \ldots \ldots$

ABSTRACT ................. ix

\section{CHAPTER}

1 Introduction ..................... 1

1.1 What are RKKY and DM interactions ............ 1

1.2 The Scope of the thesis . . . . . . . . . . . . . . . 4

2 RKKY Interaction in a Spin-Polarized Electron Gas . . . . . . 5

2.1 General formalism for RKKY and DM interactions . . . . . . 6

2.2 RKKY and DM interactions in the standard electron gas . . . . . 13

2.3 One-Dimensional Spin-Polarized Electron Gas . . . . . . . . . . . 21

2.4 Two-Dimensional Spin-Polarized Electron Gas . . . . . . . . . . 25

2.5 Three-Dimensional Spin-Polarized Electron Gas . . . . . . . . . 27

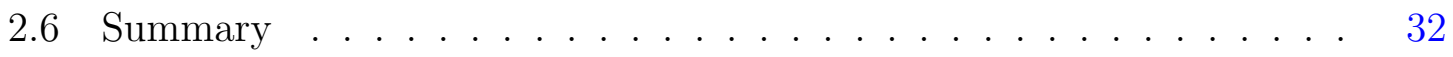

3 RKKY and DM Interactions in a Two-dimensional Electron Gas with Rashba and Dresselhaus Spin-Orbit Couplings . . . . . . 33

3.1 Formalism . . . . . . . . . . . . . . . . . . . 35

3.2 2DEG with Rashba spin-orbit coupling . . . . . . . . . . 42

3.2 .1 High Fermi-energy limit $\ldots \ldots \ldots \ldots \ldots$

3.2 .2 Low Fermi-energy limit . . . . . . . . . . . . . . . . 47 
3.3 2DEG with Dresselhaus spin-orbit coupling . . . . . . . . . . . 49

3.4 Spin-polarized 2DEG with no Rashba or Dresselhaus term . . . . . . 51

3.5 Magnetic interactions in the general case . . . . . . . . . . . 52

3.6 Domain Wall Energetics ................ 54

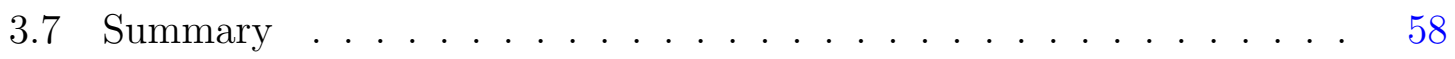

4 Impurity states in kagome lattice . . . . . . . . . . . 60

4.1 Lattice structure . . . . . . . . . . . . . . . . . . . 61

4.2 Dyson's equation and impurity states . . . . . . . . . 63

4.3 impurity state wave function . . . . . . . . . . . . 67

4.4 Conclusion . . . . . . . . . . . . . . . . . 68

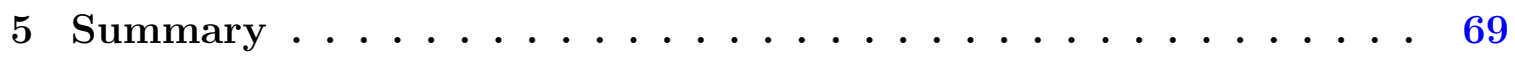

\section{APPENDIX}

A General formula for RKKY and DM interactions using LippmannSchwinger equation ................ 72

B RKKY Interaction for the Spin Polarized Electron Gas Using the Method of Summation Over Momentum . . . . . . . . . 76

B.1 Spin-polarized electron gas and magnetic interaction . . . . . . . . 79

B.2 Results........................ 85

B.2.1 Electron gas in 3D ................... 85

B.2.2 Electron Gas in 2D ................ 88

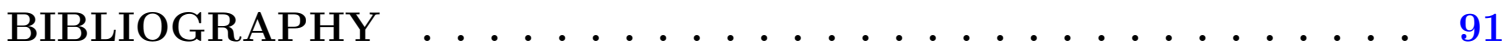

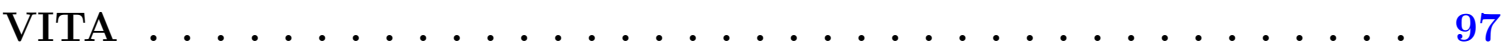




\section{LIST OF FIGURES}

Figure

1.1 The RKKY interaction between two magnetic moments $\vec{S}_{1}$ and $\vec{S}_{2}$ embedded in an electron gas. The first moment disturbs the electron gas, which is felt by the second moment. . . . . . . . . . .

2.1 Ferromagnetic and anti-ferromagnetic forms of RKKY interaction. .

2.2 Oscillatory behavior of $J$ in units of $10^{-2} \mathrm{~m}^{-1}$ as a function of $R$ in the unit of $\AA$ for given value of $k_{F}=1.2(1 / \AA)$ in a one-dimensional free electron gas. . . . . . . . . . . . . . . . . .

2.3 Oscillatory behavior of $J$ in units of $m \lambda^{2} /(8 \pi)$ as a function of $R$ in the unit of $\AA$ for given value of $k_{F}=1.2(1 / \AA)$ in a two-dimensional electron gas. . . . . . . . . . . . . . . . .

2.4 Oscillatory behavior of $J$ in unit of $m \pi^{-3} \lambda^{2}$ as a function of $R$ in the units of $\AA$ for given value of $k_{F}=1.2(1 / \AA)$ in a three-dimensional electron gas. . . . . . . . . . . . . . . . . . 
2.5 Oscillatory behaviors of $\chi_{z z}$ (right) and $\chi_{x x}$ (left) in units of $10^{-2} m \pi^{-1}$ as a function of $R$ in the units of $\AA$ for given values of $k_{F \uparrow}=1.574$ and $k_{F \downarrow}=1.3881 / \AA$. The beat-pattern oscillatory behavior of $\chi_{z z}$ can be easily understood based on two different Fermi momenta, $k_{F \uparrow}$ and $k_{F \downarrow}$, in the system. . . . . . . . . . . . . . .

2.6 Oscillatory behavior of $J_{1}$ and $J_{2}$, in the units of $10^{-5} m \lambda^{2} \pi^{-3}$ as a function of $R$ in the units of $\AA$ for the case of iron. . . . . . . . .

2.7 Oscillatory behavior of $J_{1}$ and $J_{2}$, in the units of $10^{-3} m \lambda^{2} \pi^{-3}$ as a function of $R$ in the units of $\AA$ for the case of iron. . . . . . . . .

3.1 Spin eigenfunctions for the 2DEG with Rashba spin-orbit coupling. .

3.2 Numerical results for the ratio $D_{y} / J$ and $\gamma_{y y} / J$ as a function of $\alpha$ (in $\mathrm{eV} \cdot \AA$ ) in the large $E_{F}$ and large $R$ limit. The points connected by lines indicate the numerical results, while the smooth lines are analytical results, Eqs. (3.29) - (3.31). Note from the same equations that the Fermi energy dependence drops out in the ratios, and here $R=7.5 \AA$

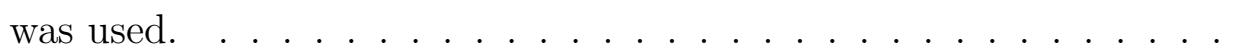

3.3 Occupied momentum states in the low- $E_{F}$ limit, valid for both cases of Rashba-only or Dresselhaus-only SOC. The occupied states form a thin circular strip, resembling a 1D electron gas. We have defined $\Delta_{k}=\left(k_{1}-k_{2}\right) / 2$ and $k_{0}=\left(k_{1}+k_{2}\right) / 2$, where $k_{0}=\alpha m / \hbar^{2}$ (Rashba) or $k_{0}=\beta m / \hbar^{2}$ (Dresselhaus). . . . . . . . . . . . . . 
3.4 The spin eigenfunctions and the Fermi surface for the case $\alpha=\beta$ and $\Delta=0$, which consists of two circles with their centers shifted by the vector $\vec{Q}=\left(Q_{x}, Q_{y}\right)=2 \sqrt{2} m \alpha(1,1)$. The two bands are indexed by $\nu=1$ for the outer shell and $\nu=2$ for the inner shell. . . . . . .

3.5 Computed magnetic interactions for equal Rashba and Dresselhaus SOC and $\Delta=0$. Here $E_{F}=3.4 \mathrm{eV}, \alpha=\beta=0.22 \mathrm{eV} \cdot \AA$, distance $R$ is in $\AA$, and the magnetic interactions are in units of $10^{-4} \times \lambda^{2} m$. . .

3.6 Right and left handed Néel and Bloch walls. . . . . . . . . . . . 56

4.1 Kagome lattice and Brillouin zone. Here $\vec{t}_{1}=a(1,0)$ and $\vec{t}_{2}=a\left(\frac{1}{2}, \frac{\sqrt{2}}{3}\right)$. For future calculations, one can use $K=\frac{4 \pi}{3 a}(1,0), K^{\prime}=-K$ and $M=\frac{2 \pi}{\sqrt{3} a}(0,1)$. Based on the symmetry of the system, all of the atoms are equivalent. . . . . . . . . . . . . . .

4.2 Tight binding band structure of the kagome lattice, with blue for the flat band and red for the dispersive bands. . . . . . . . . . . . .

4.3 Numerical results for the real part of local trace of Green's function matrix and local DOS as a function of energy. . . . . . . . . . .

4.4 Numerical results for the impurity states. As it can be seen, presence of an impurity in the system changes the density around the $K$ and

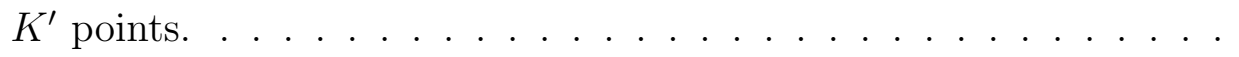


B.1 (Color online) The interaction between two magnetic moments mediated by the host electrons in the crystal, which leads to the well known RKKY interaction of the type $J \vec{S}_{1} \cdot \vec{S}_{2}$. However, if the host crystal has broken symmetry, then additional interaction terms appear, known as

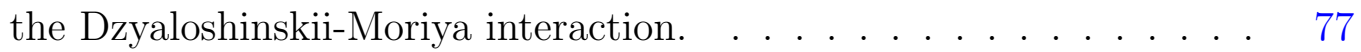

B.2 (Color online) Electron gas with spin dependent energy. $E_{F}$ denotes the Fermi energy and the Fermi momenta in the two spin channels are indicated by $k_{F \uparrow}$ and $k_{F \downarrow} \ldots \ldots \ldots \ldots \ldots \ldots$

B.3 (Color online) The RKKY interaction $J(t o p)$ and the tensor DzyaloshinskiiMoriya interaction $P$ (bottom), in units of $m \pi^{-3} \lambda^{2}$, for the 3D electron gas as a function of distance $R$, obtained from Eqs. (B.17 - B.19). $\quad . \quad 87$ 


\section{ABSTRACT}

The exchange interaction between two localized magnetic moments, $\vec{S}_{1}$ and $\vec{S}_{2}$, embedded in a host material is fundamental to the description of the magnetic behavior of solids and chiral order in magnetic structures. This interaction was originally found by Ruderman, Kittel, Kasuya and Yosida (RKKY). It is currently an area of considerable interest. The potential spintronics applications of chiral magnetic structures, originating from the competition between RKKY and Dzyaloshinsky-Moriya (DM) interactions caused by broken symmetries and the presence of the spin-orbit interaction, have stimulated lots of recent theoretical studies. In the standard spindegenerate electron gas, it leads to the well-known RKKY interaction, which has the Heisenberg form of $J \vec{S}_{1} \cdot \vec{S}_{2}$.

In this dissertation, we study RKKY and DM interactions in the spin-polarized electron gas in one, two and three dimensions. We find that an additional Ising-like term appears in the magnetic interaction, so that the net interaction for the spinpolarized gas is of the form $J_{1} \vec{S}_{1} \cdot \vec{S}_{2}+J_{2} S_{1 z} S_{2 z}$. The anisotropic interaction is due to the broken time-reversal symmetry, based on the fact that spin polarization is in the $\hat{z}$ direction.

Furthermore, we study RKKY and DM interactions in a spin-polarized twodimensional electron gas in the presence of both Rashba and Dresselhaus spin-orbit coupling (SOC). We find the net interaction to be of the form $J_{1} \vec{S}_{1} \cdot \vec{S}_{2}+\vec{D} \cdot \vec{S}_{1} \times \vec{S}_{2}+$ $\vec{S}_{1} \cdot \stackrel{\leftrightarrow}{\Gamma} \cdot \vec{S}_{2}$, where in addition to the scalar RKKY interaction, we also have the vector and tensor interactions between the two localized moments. Analytical expressions are found for each term of the total magnetic interaction, and their oscillatory be- 
haviors are studied and compared to the numerical results. We also use our model to find energy of Neel and Bloch magnetic walls. Using our theoretical results we are able to predict which wall is preferred.

In the last chapter, we study impurity states in the kagome lattice. The kagome lattice is a 2D lattice that has been of recent interest owing to its graphene-like band structure, the existence of flat band states and exotic quasiparticle excitations. Materials hosting the kagome lattice such as the rare-earth compounds and the herbertsmithites have been recently synthesized and efforts have been made to change the Fermi energy to the Dirac-like point by introducing impurities into this system. In this chapter, we study the electronic states introduced by impurities in the system by applying the Green's function approach within a tight-binding model Hamiltonian. The impurities introduce localized states close to the Dirac point, in many ways similar to graphene, which will be discussed. 


\section{Chapter 1}

\section{Introduction}

\subsection{What are RKKY and DM interactions}

The well-known RKKY interaction between two localized magnetic moments in a host solid was originally derived for the free electron gas $[1,2,3]$ and continues to be an active and important research field in different classes of materials with different symmetries $[4,5,6,7,8,9,10]$.

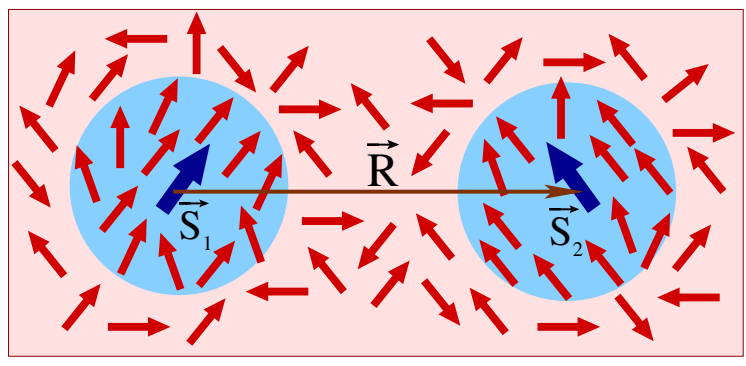

Figure 1.1: The RKKY interaction between two magnetic moments $\vec{S}_{1}$ and $\vec{S}_{2}$ embedded in an electron gas. The first moment disturbs the electron gas, which is felt by the second moment. 
For the standard free electron gas, $J$ shows an oscillatory behavior as a function of the distance $\vec{R}$ between the two moments, with the long-distance behavior $J(\vec{R}) \sim \cos \left(2 k_{F} R+(d-1) \pi / 2\right) / R^{d}$, where $k_{F}$ is the Fermi momentum and $d$ is the dimensionality of the system. The recent observation of the skyrmions in solids[11, 12, 13, 14], caused by the competition between RKKY and DzyaloshinskiiMoriya (DM) interactions, the latter originating from broken symmetry (time reversal and/or inversion), has stimulated considerable interest in systems with broken symmetry.

For a system with no broken symmetry (inversion or time reversal), this interaction has the Heisenberg form of $E(\vec{R})=J \vec{S}_{1} \cdot \vec{S}_{2}$. The spin polarized electron gas, which we consider here, is the simplest example of a system with broken symmetry, and serves to illustrate the effect of the broken symmetry on the magnetic interaction. Time reversal and inversion symmetries lead, respectively, to the conditions for the band structure energies: $\varepsilon_{\vec{k} \sigma}=\varepsilon_{-\vec{k},-\sigma}$ and $\varepsilon_{\vec{k} \sigma}=\varepsilon_{-\vec{k} \sigma}$, where $\sigma=\uparrow$ or $\downarrow$, and $-\sigma$ denotes opposite of $\sigma$. If both symmetries are present, then these conditions together lead to the spin-degenerate energies in the band structure, viz., $\varepsilon_{\vec{k} \sigma}=\varepsilon_{\vec{k},-\sigma}$. Note that for the unpolarized electron gas, both symmetries are present, so that the bands are spin degenerate, while for the spin-polarized electron gas, the inversion symmetry is present, but the time reversal symmetry is broken. For the Rashba spin-orbit interaction [15], the reverse is true: That is, the electric field present there breaks the inversion symmetry, while the time reversal symmetry is still present.

Broken symmetries, break this degeneracy of the energy bands, change the Green's function matrix, and finally lead to extra terms in the interaction between two localized magnetic moments. Using lattice models, Dzyaloshinskii and Moriya showed 
that $[16,17]$ in certain situations with broken inversion symmetry, the net interaction is given by the expression:

$$
E(\vec{R})=J(\vec{R}) \vec{S}_{1} \cdot \vec{S}_{2}+\vec{D}(\vec{R}) \cdot \vec{S}_{1} \times \vec{S}_{2}+\vec{S}_{1} \cdot \stackrel{\leftrightarrow}{\Gamma} \cdot \vec{S}_{2}
$$

where in addition to the scalar RKKY-type interaction, we also have the vector and tensor interactions between the two localized moments $\vec{S}_{1}$ and $\vec{S}_{2}$.

Studying of the RKKY and DM interactions in a host solid with broken symmetries plays a crucial role to understand the physics of the magnetic chiral ordering and Skyrmions $[11,12,13,14]$.

It is important to mention the fact that here we neglected the classical magnetic dipole-dipole interaction between two localized magnetic moments. Magnetic dipole-dipole interaction, also known as dipolar coupling, is the direct interaction between two magnetic dipoles, and has the form of [18]

$$
E_{d}(\vec{R})=-\frac{\mu_{0} \hbar^{2} \gamma_{1} \gamma_{2}}{4 \pi r^{3}}\left(3\left(\vec{S}_{1} \cdot \hat{r}\right)\left(\vec{S}_{2} \cdot \hat{r}\right)-\vec{S}_{1} \cdot \vec{S}_{2}\right)
$$

where $\gamma_{1}$ and $\gamma_{2}$ are gyromagnetic ratios of two particles with spin $\vec{S}_{1}$ and $\vec{S}_{2}$ (in the unit of $1 / 2$, since $\hbar$ has been already taken into account), $\mu_{0}$ is the magnetic constant $\hat{r}$ is a unit vector in the direction of the line joining the two spins, and $|r|$ is the distance between them [18]. Here we assumed this interaction is weak comparing to RKKY and Dzyaloshinskii-Moriya interactions. Taking $\gamma_{1} \simeq \gamma_{2} \simeq 1, \hbar=1.05 \times 10^{-34}$, $\mu=4 \pi \times 10^{-7}, r=10^{-10}$ and $S_{1}$ and $S_{2}$ as one (or $1 / 2$ ), one immediately finds that the order of this interaction is almost $10^{-19} e \cdot V$, much smaller than RKKY and DM interactions. 


\subsection{The Scope of the thesis}

The plan of this thesis is as follows. Chapter 2 is devoted to the study of RKKY and DM interactions in a more general case of spin-polarized electron gas. We find analytical expressions and study oscillatory behavior of them in one- , two- and threedimensional spin polarized electron gas.

In chapter 3 , the spin-orbit coupling (SOC) comes to the picture. We show that Rashba and Dresselhaus SOC will play an important role for strength of DM interaction, and therefore in creation of chiral magnetic ordering. In this chapter, we also study two basic forms of the magnetic walls, Neel and Bloch magnetic walls. We use our results to find the energy of each wall and then using it, we predict which one is preferred. In Chapter 4, we use the Green's function method to study effect of putting an impurity on the density of states of kagome lattice. The kagome lattice is a $2 \mathrm{D}$ lattice that has been of recent interest owing to its graphene-like band structure, the existence of flat band states and exotic quasiparticle excitations. We find analytical form of Green's function matrix, which can be used to find RKKY and DM interactions in kagome lattice.

In the appendices, we first provide another method to find the general formula for RKKY and DM interactions. We also derive the result of RKKY and DM interactions found in chapter 2 using another method. 


\section{Chapter 2}

\section{RKKY Interaction in a Spin-Polarized Electron Gas}

We study the indirect exchange interaction between two localized magnetic moments, known as Ruderman-Kittel-Kasuya-Yosida (RKKY) interaction, in a one-, two- and three-dimensional spin-polarized electron gas. We first derive a general formula for the interaction between two localized moments in a solid and then we study this interaction in a standard electron gas. After that we will do the calculations for the case of spin-polarized electron gas and find explicit expressions for each term of this interaction, study their oscillatory behaviors as a function of the distance between two magnetic moments, $R$, and compare them with the known results for RKKY interaction in the case of one-dimensional standard electron gas. We show this interaction can be written in an anisotropic Heisenberg form, $E(\vec{R})=\lambda^{2} \chi_{x x}\left(S_{1 x} S_{2 x}+S_{1 y} S_{2 y}\right)+\lambda^{2} \chi_{z z} S_{1 z} S_{2 z}$, coming from broken time-reversal symmetry of the host material. 


\subsection{General formalism for RKKY and DM inter- actions}

In this section, we derive the general expression for the magnetic interaction, Eq. (2.19), from the second order perturbation theory, which is somewhat more pedagogical than using other methods. The same equation has been derived using LippmannSchwinger equation[19] in the Appendix.

Let $\psi_{\vec{k} \nu}(\vec{r} \sigma)$ denote the host electron wave functions $\left(|\vec{k} \nu\rangle=\sum_{\vec{r} \sigma} \psi_{\vec{k} \nu}(\vec{r} \sigma)|\vec{r} \sigma\rangle\right.$ in Dirac notations), where $k \nu$ are the quantum numbers (e. g., Bloch momentum $k$ and band index $\nu$ in a crystal) and $\varepsilon_{k \nu}$ be the corresponding eigenenergies. The interaction between the two localized moments $\vec{S}_{1}$ and $\vec{S}_{2}$ (located at origin and $\vec{R}$, respectively) and the host electrons are taken, as usual, to be the contact interactions: $V_{1}(\vec{r})=-\lambda \delta(\vec{r}) \vec{S}_{1} \cdot \vec{s}$ and $V_{2}(\vec{r})=-\lambda \delta(\vec{r}-\vec{R}) \vec{S}_{2} \cdot \vec{s}$. According to the second-order perturbation theory, the change of energy due to this interaction is given by the equation

$$
E(\vec{R})=\sum_{\vec{k} \nu}^{o c c} \sum_{\overrightarrow{k^{\prime}} \nu^{\prime}}^{\prime} \frac{\left|\left\langle\vec{k} \nu\left|\hat{V}_{1}+\hat{V}_{2}\right| \overrightarrow{k^{\prime}} \nu^{\prime}\right\rangle\right|^{2}}{\varepsilon_{\vec{k} \nu}-\varepsilon_{\overrightarrow{k^{\prime}} \nu^{\prime}}},
$$

where the prime over the summation indicates that the term $k^{\prime} \nu^{\prime}=k \nu$ is excluded and the interactions are in the operator forms, viz., $\hat{V}_{1}=-\lambda \sum_{\sigma \sigma^{\prime}}|\overrightarrow{0} \sigma\rangle \vec{S}_{1} \cdot \vec{s}\left\langle\overrightarrow{0} \sigma^{\prime}\right|$ and $\hat{V}_{2}=-\lambda \sum_{\sigma \sigma^{\prime}}|\vec{R} \sigma\rangle \vec{S}_{2} \cdot \vec{s}\left\langle\vec{R} \sigma^{\prime}\right|$. It is convenient to write the energy expression Eq. (2.1) in terms of the retarded and advanced Green's functions, $\hat{G}(E)=(E+i \mu-\hat{H})^{-1}$ and $\hat{G}^{A}(E)=(E-i \mu-\hat{H})^{-1}$, where $\mu \rightarrow 0^{+}$. With the use of the identity

$$
P\left(\frac{1}{x}\right)=\lim _{\mu \rightarrow 0^{+}} \frac{1}{2}\left(\frac{1}{x+i \mu}+\frac{1}{x-i \mu}\right)
$$


where $P$ denotes the principal part, and the expression for the Green's function

$$
\hat{G}(E)=\sum_{\vec{k} \nu} \frac{|\vec{k} \nu\rangle\langle\vec{k} \nu|}{E-\varepsilon_{\vec{k} \nu}+i \mu}
$$

one finds after some algebra, the result

$$
E(\vec{R})=\sum_{\vec{k} \nu}^{o c c}\left\langle\vec{k} \nu\left|\hat{V}_{2} \hat{G}\left(\varepsilon_{\vec{k} \nu}\right) \hat{V}_{1}\right| \vec{k} \nu\right\rangle+\text { h.c. }
$$

where the extra terms $\left(\overrightarrow{k^{\prime}} \nu^{\prime}=\vec{k} \nu\right)$ added to Eq. (2.1) to write in terms of the Green's functions add up to zero. Note that only the cross terms in the interactions $\hat{V}_{1}$ and $\hat{V}_{2}$ have been kept, since only these depend on $\vec{R}$.

Alternatively, the above expression for $E(\vec{R})$, Eq. (2.4), may also be obtained from the Lippmann-Schwinger equation[19]. Based on the importance of this equation, we provided its derivation using Lippmann-Schwinger equation in the Appendix. According to this equation, the host electron states become modified by the perturbation $\hat{V}_{1}$ to the state $|\widetilde{\vec{k}} \nu\rangle$

$$
|\widetilde{\vec{k}} \nu\rangle=|\vec{k} \nu\rangle+\hat{G} \hat{V}_{1}|\vec{k} \nu\rangle
$$

where we have kept terms correct to the first order in the perturbation theory. The second localized moment feels this perturbation, so that the interaction energy is given by

$$
\begin{aligned}
E(\vec{R}) & =\sum_{\vec{k} \nu}^{o c c}\left\langle\widetilde{\vec{k}} \nu\left|\hat{V}_{2}\right| \widetilde{\vec{k}} \nu\right\rangle \\
& =\sum_{\vec{k} \nu}^{o c c}\left\langle\vec{k} \nu\left|\left(1+\hat{V}_{1} \hat{G}^{\dagger}\right) \hat{V}_{2}\left(1+\hat{G} \hat{V}_{1}\right)\right| \vec{k} \nu\right\rangle,
\end{aligned}
$$


which is the same as Eq. (2.4), when as usual we keep only the $\vec{R}$-dependent terms.

We now proceed to simplify Eq. (2.4) and express it as a Trace over product of the Green's function matrices. Using the completeness relation $\sum_{\vec{r} \sigma}|\vec{r} \sigma\rangle\langle\vec{r} \sigma|=1$, Eq. (2.4) leads to

$$
\begin{aligned}
E(\vec{R})= & \lambda^{2} \sum_{\vec{k} \nu}^{o c c} \sum_{\sigma_{1} \sigma_{2}}\left\langle\sigma_{1}\left|\vec{S}_{2} \cdot \vec{s} G\left(\vec{R}, 0, \varepsilon_{\vec{k} \nu}\right) \vec{S}_{1} \cdot \vec{s}\right| \sigma_{2}\right\rangle \\
& \times \psi_{\vec{k} \nu}^{*}\left(\vec{R}, \sigma_{1}\right) \psi_{\vec{k} \nu}\left(0, \sigma_{2}\right)+\text { h.c. }
\end{aligned}
$$

where $G_{\sigma_{1} \sigma_{2}}\left(\vec{r}_{1}, \vec{r}_{2}, E\right) \equiv\left\langle\vec{r}_{1} \sigma_{1}|\hat{G}(E)| \vec{r}_{2} \sigma_{2}\right\rangle$ is given by

$$
G_{\sigma \sigma^{\prime}}\left(\vec{r}_{1}, \vec{r}_{2}, E\right)=\sum_{\vec{k} \nu} \frac{\psi_{\vec{k} \nu}\left(\vec{r}_{1}, \sigma\right) \psi_{\vec{k} \nu}^{*}\left(\vec{r}_{2}, \sigma^{\prime}\right)}{E+i \mu-\varepsilon_{\vec{k} \nu}} .
$$

Expressing the Green's function as an integral over energy

$$
G\left(\vec{R}, 0, \varepsilon_{\vec{k} \nu}\right)=\int G(\vec{R}, 0, E) \delta\left(E-\varepsilon_{\vec{k} \nu}\right) d E
$$

and the fact that $\int_{-\infty}^{\infty} d E \times \sum_{\vec{k} \nu} \rightarrow \int_{-\infty}^{E_{F}} d E \times \sum_{\vec{k} \nu}^{o c c}$, Eq. (2.7) leads to the result

$$
\begin{aligned}
E(\vec{R})= & \lambda^{2} \int_{-\infty}^{E_{F}} d E \sum_{\sigma_{1} \sigma_{2}}\left\langle\sigma_{1}\left|\vec{S}_{2} \cdot \vec{s} G(\vec{R}, 0, E) \vec{S}_{1} \cdot \vec{s}\right| \sigma_{2}\right\rangle \\
& \times \sum_{\vec{k} \nu} \psi_{\vec{k} \nu}^{*}\left(\vec{R}, \sigma_{1}\right) \psi_{\vec{k} \nu}\left(0, \sigma_{2}\right) \delta\left(E-\varepsilon_{\vec{k} \nu}\right)+h . c .
\end{aligned}
$$

The second line can be expressed as the difference between the retarded and the 
advanced Green's function, viz.,

$$
\begin{aligned}
& \sum_{\vec{k} \nu} \psi_{\vec{k} \nu}^{*}\left(\vec{R}, \sigma_{1}\right) \psi_{\vec{k} \nu}\left(0, \sigma_{2}\right) \delta\left(E-\varepsilon_{\vec{k} \nu}\right) \\
= & \frac{i}{2 \pi}\left[G(0, \vec{R}, E)-G^{A}(0, \vec{R}, E)\right]_{\sigma_{2} \sigma_{1}},
\end{aligned}
$$

since $\lim _{\mu \rightarrow 0^{+}}(x \pm i \mu)^{-1}=P\left(x^{-1}\right) \mp i \pi \delta(x)$, so that

$$
\delta\left(E-\varepsilon_{\vec{k} \nu}\right)=\frac{i}{2 \pi}\left(\frac{1}{E+i \mu-\varepsilon_{\vec{k} v}}-\frac{1}{E-i \mu-\varepsilon_{\vec{k} v}}\right) .
$$

From Eqs. (2.10) and (2.11), we find

$$
\begin{aligned}
E(\vec{R})= & \frac{-\lambda^{2}}{\pi} \operatorname{Im} \int_{-\infty}^{E_{F}} \operatorname{Tr}\left[G(0, \vec{R}, E) \vec{S}_{2} \cdot \vec{s}\right. \\
& \left.G(\vec{R}, 0, E) \vec{S}_{1} \cdot \vec{s}\right] d E .
\end{aligned}
$$

This is the desired result of the section.

We left out in Eq. (2.13), the term involving $G^{A}$, since it turns out to be zero, i. e.,

$$
\begin{gathered}
I=\frac{\lambda^{2}}{\pi} \operatorname{Im} \int_{-\infty}^{E_{F}} \operatorname{Tr}\left[G^{A}(0, \vec{R}, E) \vec{S}_{2} \cdot \vec{s}\right. \\
\left.G(\vec{R}, 0, E) \vec{S}_{1} \cdot \vec{s}\right] d E=0 .
\end{gathered}
$$

This is readily shown by expanding the Green's functions in terms of the Pauli ma- 
trices:

$$
\begin{aligned}
& G(\vec{R}, 0, E)=g_{0} \sigma_{0}+\sum_{i=1}^{3} g_{i} \sigma_{i}, \\
& G^{A}(0, \vec{R}, E)=G^{\dagger}(\vec{R}, 0, E)=g_{0}^{*} \sigma_{0}+\sum_{i=1}^{3} g_{i}^{*} \sigma_{i},
\end{aligned}
$$

and using the trace equalities

$$
\begin{aligned}
& \operatorname{Tr}(A+B)=\operatorname{Tr} A+\operatorname{Tr} B, \\
& \operatorname{Tr}\left(\sigma_{i} \sigma_{j}\right)=2 \delta_{i j}, \\
& \operatorname{Tr}\left(\sigma_{i} \sigma_{j} \sigma_{k}\right)=2 i \varepsilon_{i j k}, \\
& \operatorname{Tr}\left(\sigma_{i} \sigma_{j} \sigma_{k} \sigma_{l}\right)=2\left(\delta_{i j} \delta_{k l}-\delta_{i k} \delta_{j l}+\delta_{i l} \delta_{j k}\right),
\end{aligned}
$$

written in terms of the Kronecker deltas $\delta_{i j}$ and the Levi-Civita symbols $\varepsilon_{i j k}$.

As we said, we take the localized moments to interact with the host electrons, described by the Hamiltonian $\hat{H}$, via the contact interaction

$$
V_{1}(\vec{r})=-\lambda \delta(\vec{r}) \vec{S}_{1} \cdot \vec{s}
$$

and

$$
V_{2}(\vec{r})=-\lambda \delta(\vec{r}-\vec{R}) \vec{S}_{2} \cdot \vec{s}
$$

where $\vec{s}$ is the spin of the electron, $\vec{S}_{1}$ and $\vec{S}_{2}$ are the two localized moments located at the origin and at $\vec{R}$, respectively, $\vec{r}$ is the position of the electron, the $\delta$ functions indicate local interactions at the site of the localized moments, and, finally, $\lambda$ is the strength of the electron interaction with the local moments. 
Now if we use the final result we found for the interaction in this section (also a pedagogical derivation is given in the Appendix), viz.,

$$
\begin{aligned}
E(\vec{R})= & \frac{-\lambda^{2}}{\pi} \operatorname{Im} \int_{-\infty}^{E_{F}} \operatorname{Tr}\left[G(0, \vec{R}, E) \vec{S}_{2} \cdot \vec{s}\right. \\
& \left.G(\vec{R}, 0, E) \vec{S}_{1} \cdot \vec{s}\right] d E
\end{aligned}
$$

where $\hat{G}(E)=(E+i \eta-\hat{H})^{-1}$ with $\eta \rightarrow 0^{+}$, is the retarded Green's function. The matrix elements are given by

$$
G_{\sigma_{1} \sigma_{2}}\left(\vec{r}_{1}, \vec{r}_{2}, E\right)=\sum_{\vec{k} \nu}^{\infty} \frac{\psi_{\vec{k} \nu}\left(\vec{r}_{1}, \sigma_{1}\right) \psi_{\vec{k} \nu}^{*}\left(\vec{r}_{2}, \sigma_{2}\right)}{E+i \eta-\varepsilon_{\vec{k} \nu}}
$$

where $G_{\sigma_{1} \sigma_{2}}\left(\vec{r}_{1}, \vec{r}_{2}, E\right) \equiv\left\langle\vec{r}_{1} \sigma_{1}|\hat{G}(E)| \vec{r}_{2} \sigma_{2}\right\rangle, \psi_{\vec{k} \nu}(\vec{r}, \sigma)=\langle\vec{r} \sigma \mid \vec{k} \nu\rangle$, and $\vec{k} \nu$ labels the eigenstates of the system.

Working through the derivation of Eq. (2.19), given in chapter 1 and the Appendix, provides some important information: to get the resutl as Eq. (2.19), one needs to change the order of integration between $E$ and $k$, which can lead to a different result for the double-intergral when Fubini's condition [20, 21] is not satisfied. Using Eq. (2.19) to find the RKKY interaction in a one-dimensional electron gas leads to the wrong result, and is a simple example for this case [22, 23, 24].

In the present case, the Green's function is diagonal in the spin indices, because the eigenstates in the spin-polarized electron gas are either spin up or spin down. This is not generally true, if the eigenstates are mixed spin states, e. g., in the case of electron gas with Rashba interaction [15].

The eigenstates are in general mixed-spin, but in the present case they are pure- 
spin states, so that the Green's function is diagonal in the spin indices. Furthermore, if the wave functions can be chosen to be real (true if $\psi_{\vec{k} \nu}(\vec{r}, \sigma)$ and $\psi_{\vec{k} \nu}^{*}(\vec{r}, \sigma)$ are solutions with the same energy), then it follows from Eq. (2.20) that $G_{\sigma_{1} \sigma_{2}}\left(\vec{r}_{1}, \vec{r}_{2}, E\right)=$ $G_{\sigma_{2} \sigma_{1}}\left(\vec{r}_{2}, \vec{r}_{1}, E\right)$. It follows then that, since the Green's function are spin diagonal, we have the equality $G(0, \vec{R}, E)=G(\vec{R}, 0, E)$.

Under these conditions, we can expand the Green's function matrix in terms of the Pauli matrices $\vec{\sigma}$ as

$$
G(0, \vec{R}, E)=G(\vec{R}, 0, E)=g_{0}(E) \sigma_{0}+g_{z}(E) \sigma_{z},
$$

$\sigma_{0}$ being the unit $2 \times 2$ matrix. Note that in order to describe the spin-diagonal Green's function in Eq. (2.21), we need to use $\sigma_{z}$, which means that we have effectively chosen $\hat{z}$ as the spin quantization axis and this is why the $z$ components $S_{1 z}$ and $S_{2 z}$ appear in Eq. (2.23) that follows. Using now Eq. (2.21), the energy expression Eq. (2.19) can be evaluated using the following spin identities

$$
\begin{aligned}
& \operatorname{Tr}\left[\vec{S}_{2} \cdot \vec{\sigma} \vec{S}_{1} \cdot \vec{\sigma}\right]=2 \vec{S}_{1} \cdot \vec{S}_{2}, \\
& \operatorname{Tr}\left[\vec{S}_{2} \cdot \vec{\sigma} \sigma_{z} \overrightarrow{S_{1}} \cdot \vec{\sigma}\right]=2 i\left(\vec{S}_{1} \times \vec{S}_{2}\right)_{z}, \\
& \operatorname{Tr}\left[\sigma_{z} \vec{S}_{2} \cdot \vec{\sigma} \sigma_{z} \vec{S}_{1} \cdot \vec{\sigma}\right]=-2 \vec{S}_{1} \cdot \vec{S}_{2}+4 S_{1 z} S_{2 z}
\end{aligned}
$$

The result is

$$
E(\vec{R})=J_{1} \vec{S}_{1} \cdot \vec{S}_{2}+J_{2} S_{1 z} S_{2 z}
$$


where

$$
\begin{aligned}
& J_{1}=\frac{-\lambda^{2} \hbar^{2}}{2 \pi} \times \operatorname{Im} \int_{-\infty}^{E_{F}}\left(g_{0}^{2}-g_{z}^{2}\right) d E \\
& J_{2}=\frac{-\lambda^{2} \hbar^{2}}{2 \pi} \times \operatorname{Im} \int_{-\infty}^{E_{F}} 2 g_{z}^{2} d E
\end{aligned}
$$

These expressions can be evaluated from the Green's functions, which we now proceed to do for the spin-polarized electron gas in 1D, 2D and 3D. Note from Eq. (2.24) that for the standard (spin unpolarized) electron gas, the Green's function has equal diagonal elements, so that $g_{z}=0$, and the $J_{2}$ terms vanishes as a result and one obtains the standard $\vec{S}_{1} \cdot \vec{S}_{2}$ RKKY interaction.

\subsection{RKKY and DM interactions in the standard electron gas}

For the case of standard electron gas, both inversion and time-reversal symmetries are present and it will lead to $G(\vec{R}, 0, E)=G(0, \vec{R}, E)=g \sigma_{0}$. Using this fact in Eq. (2.13), leads to RKKY interaction as $J(|\vec{R}|) \vec{S}_{1} \cdot \vec{S}_{2}$ where $J(|\vec{R}|)$ is defined as

$$
J(|\vec{R}|)=\frac{\hbar^{2} \lambda^{2}}{2 \pi} \int_{-\infty}^{E_{F}} \operatorname{Im}\left[g^{2}\right] d E .
$$

As it can be seen, DM interaction vanishes for standard free electron gas. This result could be expected from the fact that inversion symmetry is present. We will discuss effect of broken inversion symmetry in chapter three of this thesis.

Discussions - As it was mentioned, for the case of standard electron gas, RKKY interaction will have the form of $J(|\vec{R}|) \vec{S}_{1} \cdot \vec{S}_{2}$, which leads to the fact that when 


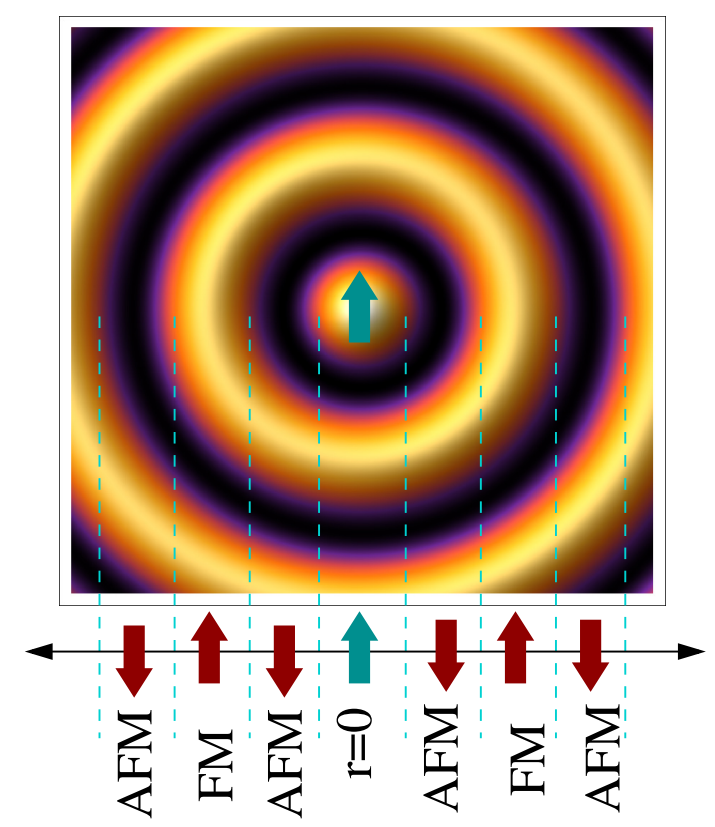

Figure 2.1: Ferromagnetic and anti-ferromagnetic forms of RKKY interaction.

$J(|\vec{R}|)<0$, the localized magnetic moments would like to have ferromagnetic states and when $J(|\vec{R}|)>0$, the interaction will be anti-ferromagnetic. Since sign of $J(|\vec{R}|)$ will change by changing the distance between two localized moments, by changing the distance RKKY interaction will be changing between ferromagnetic and antiferromagnetic phases as shown in Fig (2.1).

To find $J(|\vec{R}|)$ using Eq. (2.25), one just needs to find Green's function (GF), $G(\vec{R}, 0, E)$. So the first step will be finding GF matrix. We will use Eq. (2.8) to find GF matrix in one-, two- and three-dimensional electron gas. Then we will find RKKY interaction in each case. Straightforward calculations lead to the oscillatory Heisenberg form of RKKY interaction, $J \vec{S}_{1} \cdot \vec{S}_{2}$, in a two- and three-dimensional electron gas, but the result which provides the correct answer to the same problem in a one-dimensional (1D) electron gas was always challenging $[22,23,24,30]$ due to the dependence of the double intergral on the order of integration. Similar difficulties 
appear for the case of the one-dimensional spin polarized electron gas.

One-dimensional electron gas: For the case of one-dimensional electron gas the unperturbed eigenstates and energy bands are defined as $|\vec{k} \sigma\rangle=\frac{1}{\sqrt{L}} e^{i \vec{k} \cdot \vec{r}}|\sigma\rangle$ and $\varepsilon_{\vec{k} \sigma}=\frac{\hbar^{2} k^{2}}{2 m}$. To find the elements of Green's function matrix, one can use Eq. (2.8) and find the result

$$
G\left(\vec{r}_{1}, \vec{r}_{2}, E\right)=-\frac{i m}{\hbar^{2}}\left(\begin{array}{cc}
\frac{e^{i \alpha(E) r}}{\alpha(E)} & 0 \\
0 & \frac{e^{i \alpha(E) r}}{\alpha(E)}
\end{array}\right)
$$

where

$$
\alpha(x)=\left\{\begin{array}{l}
\left(2 m \hbar^{-2} x\right)^{1 / 2} \text { if } x>0, \\
i\left(2 m \hbar^{-2}|x|\right)^{1 / 2} \text { if } x<0 .
\end{array}\right.
$$

Discussions - Here, we would like to emphasize using Eq. (2.19) with the Green's function matrices found in Eq. (2.26)) leads to the wrong unphysical results for standard one-dimensional electron gas, viz.,

$$
J=\frac{\lambda^{2} m}{2 \pi} \operatorname{Si}\left(2 k_{F} R\right)
$$

where $\operatorname{Si}(x)$ is the sine intergral,

$$
\operatorname{Si}(x)=\int_{0}^{x} \frac{\sin (t)}{t} d t
$$

In a similar work [5], Imamura et al. have used the same method. To obtain physical answer to the problem, the authors inserted a constant, $-\lambda^{2} m / 2 \pi$ found by Yafet [22], to the last result. Yafet explained [22] that the strong singularities in the 
intergrant of double integral is responsible for the dependence of the double integral on the order of integration. Adding this constant to last result for $J$ leads to the right answer to the problem. On the other hand, Giuliani and coauthors[23] provided a very pedagogical method to obtain the right answer to the problem. This method is based on finding the delocalized eigenstates of the one-dimensional Schrodinger equation with a delta-fucntion as the potential, $V(x)=u \delta(x)$ when $u \rightarrow 0^{+}$, and then using them to find the electronic density. Similarly, one can use these eigenstates to find the full Green's function of the system as $G_{F}\left(r, r^{\prime}, E\right)=g\left(r, r^{\prime}, E\right) \sigma_{0}$, where

$$
\begin{aligned}
g\left(r, r^{\prime}, E\right) & =\frac{1}{2 \pi}\left\{\int_{0}^{\infty} \frac{\cos \left(\mathrm{k}\left|\mathrm{r}-\mathrm{r}^{\prime}\right|\right)}{E+i \mu-\frac{\hbar^{2} k^{2}}{2 m}} d k\right. \\
& -\int_{0}^{\infty} \frac{2 u^{2}}{4 k^{2}+u^{2}} \frac{\cos \left(\mathrm{k}\left|\mathrm{r}+\mathrm{r}^{\prime}\right|\right)}{E+i \mu-\frac{\hbar^{2} k^{2}}{2 m}} d k \\
& \left.+\int_{0}^{\infty} \frac{4 k u}{4 k^{2}+u^{2}} \frac{\sin \left(\mathrm{k}\left|\mathrm{r}+\mathrm{r}^{\prime}\right|\right)}{E+i \mu-\frac{\hbar^{2} k^{2}}{2 m}} d k\right\} .
\end{aligned}
$$

The last result is valid for any value of $u$. Now using the full and unperturbed Green's function matrices, it is convenient to find the change in the local electronic density, $\delta n(x)=\frac{-1}{\pi} \operatorname{Im} \int_{0}^{E_{F}} \operatorname{Tr}\left[G_{F}(x, x, E)-G(x, x, E)\right] d E$, for the case that $u \rightarrow 0^{+}$. The result provides the right answer to the problem, viz.

$$
\delta n(x)=-\frac{u}{\pi} \operatorname{si}\left(2 k_{F}|x|\right),
$$

where $\operatorname{si}(x)=\operatorname{Si}(x)-\frac{\pi}{2}$. To get the last result, one may need to use the following 


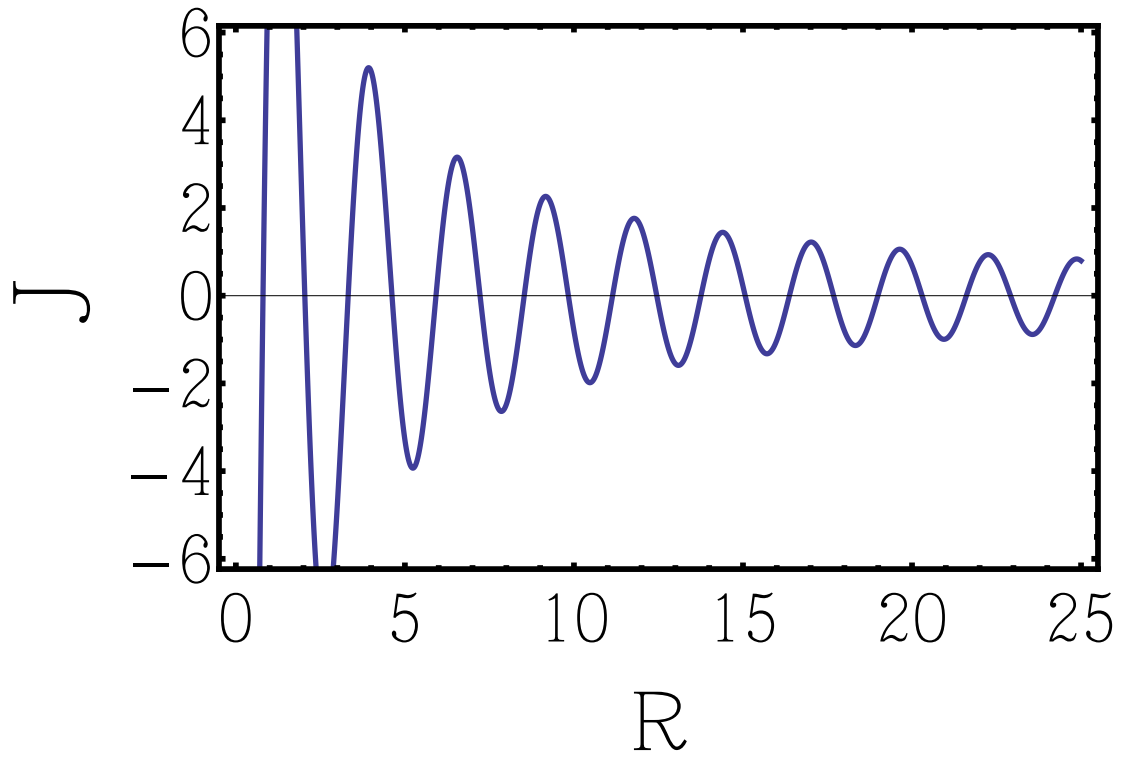

Figure 2.2: Oscillatory behavior of $J$ in units of $10^{-2} m \pi^{-1}$ as a function of $R$ in the unit of $\AA$ for given value of $k_{F}=1.2(1 / \AA)$ in a one-dimensional free electron gas.

Dirac delta function equalities

$$
\begin{gathered}
\delta(k)=\frac{2}{\pi} \lim _{u \rightarrow 0^{+}} \frac{u}{4 k^{2}+u^{2}}, \\
\delta(E)=-\frac{1}{\pi} \lim _{\mu \rightarrow 0^{+}} \operatorname{Im}\left[\frac{1}{E+i \mu}\right], \\
\delta\left(k^{2}-\frac{2 m E}{\hbar^{2}}\right)=\sqrt{\frac{\hbar^{2}}{8 m E}}\left\{\delta\left(k-\sqrt{\frac{2 m E}{\hbar^{2}}}\right)+\delta\left(k+\sqrt{\frac{2 m E}{\hbar^{2}}}\right)\right\} .
\end{gathered}
$$

We illustrate the oscillatory behavior of $J$ in Fig. (2.2) for typical parameters. Similar methods can be used to find the magnetic interaction between two localized moments in a spin-polarized one-dimensional electron gas.

Two-dimensional electron gas: The Green's function for the spin polarized 
electron gas in $2 \mathrm{D}$ is given by

$$
G_{\sigma \sigma^{\prime}}\left(\vec{r}, \vec{r}^{\prime}, E\right)=\frac{\delta_{\sigma \sigma^{\prime}}}{(2 \pi)^{2}} \int \frac{e^{i \vec{k} \cdot\left(\vec{r}-\vec{r}^{\prime}\right)}}{E+i \eta-\varepsilon_{\vec{k} \sigma}} d^{2} k
$$

with $\varepsilon_{\vec{k} \sigma}=\frac{\hbar^{2} k^{2}}{2 m}$. This is a standard integration, which can be evaluated by using the Jacobi-Anger expansion of the exponential term in terms of the Bessel's functions and by performing the angular integration. $[25,26]$ The result is

$$
G_{\sigma \sigma}\left(\vec{r}, \vec{r}^{\prime}, E\right)=g\left(r, r^{\prime}, E\right) \sigma_{0}=-\frac{m}{\pi \hbar^{2}} K_{0}\left[-i \frac{\sqrt{2 m}}{\hbar}\left|\vec{r}-\vec{r}^{\prime}\right| \alpha(E)\right]
$$

where $K_{0}$ is the modified Bessel function of the second kind, and

$$
\alpha(x)= \begin{cases}\sqrt{x} & \text { if } x>0 \\ i \sqrt{|x|} & \text { if } x<0\end{cases}
$$

To find the imaginary and the real parts of the modified Bessel function, it is convenient to use the equality $K_{\nu}(x)=2^{-1} \pi i^{\nu+1} H_{\nu}^{1}(i x)$, which is valid for $-\pi<\arg (x) \leq$ $\pi / 2$. The Hankel function of the first kind is written in terms of the Bessel and Neumann functions as $H_{\nu}{ }^{1}(x)=J_{\nu}(x)+i Y_{\nu}(x)$. Plugging it into Eq. (2.25), we find the results

$$
J=\frac{\lambda^{2} m}{8 \pi R^{2}} I\left(\frac{\sqrt{2 m E_{F}}}{\hbar} R\right)
$$

where $I(x)=x^{2}\left[J_{0}(x) Y_{0}(x)+J_{1}(x) Y_{1}(x)\right]$.

We studied the oscillatory behavior of $J$ in Fig. (2.3) for typical parameters.

Three-dimensional electron gas: Our starting point is the electron band 


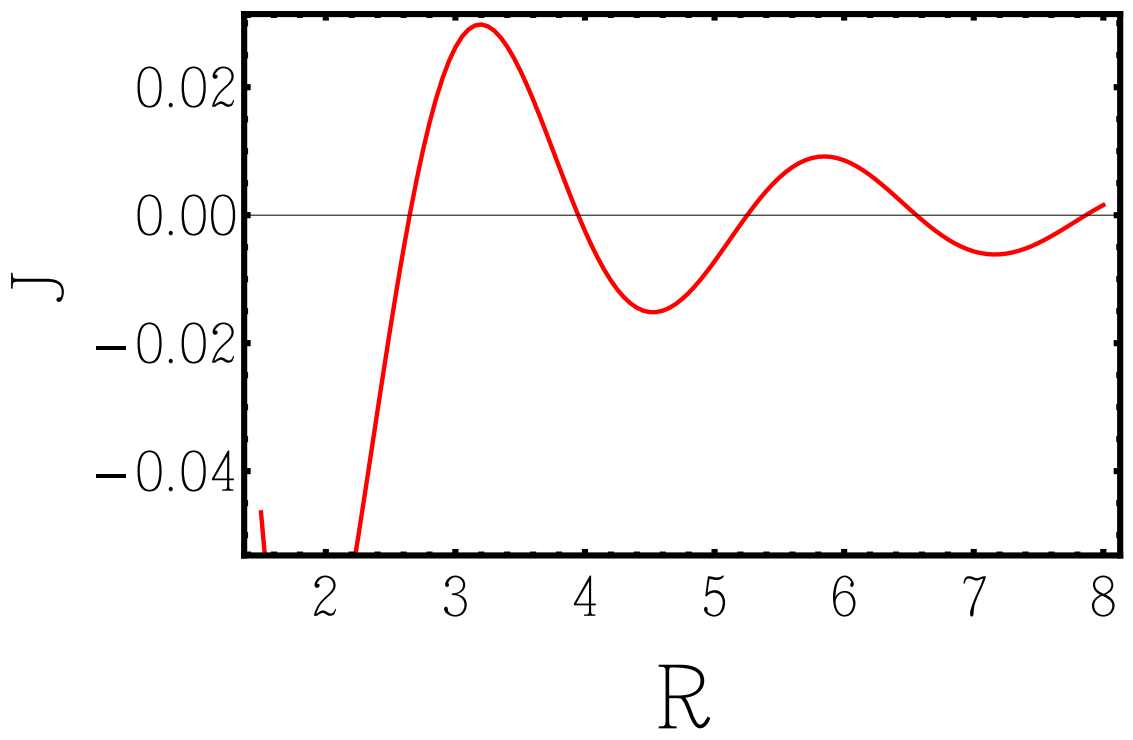

Figure 2.3: Oscillatory behavior of $J$ in units of $m \lambda^{2} /(8 \pi)$ as a function of $R$ in the unit of $\AA$ for given value of $k_{F}=1.2(1 / \AA)$ in a two-dimensional electron gas.

structure

$$
\varepsilon_{\vec{k} \sigma}=\frac{\hbar^{2} k^{2}}{2 m}
$$

and the corresponding plane-wave eigenstates are

$$
|\vec{k} \sigma\rangle=\frac{1}{\sqrt{\Omega}} e^{i \vec{k} \cdot \vec{r}}|\sigma\rangle
$$

where the $\Omega$ is the volume of the box for normalization.

The key quantity to evaluate is the Green's function, which, using Eqs. (2.8), (2.37), and (2.38) and converting the summation into integration in the momentum space, is written as

$$
G_{\sigma \sigma^{\prime}}\left(\vec{r}, \vec{r}^{\prime}, E\right)=\frac{\delta_{\sigma \sigma^{\prime}}}{(2 \pi)^{3}} \int \frac{e^{i \vec{k} \cdot\left(\vec{r}-\vec{r}^{\prime}\right)}}{E+i \eta-\varepsilon_{\vec{k} \sigma}} d^{3} k .
$$




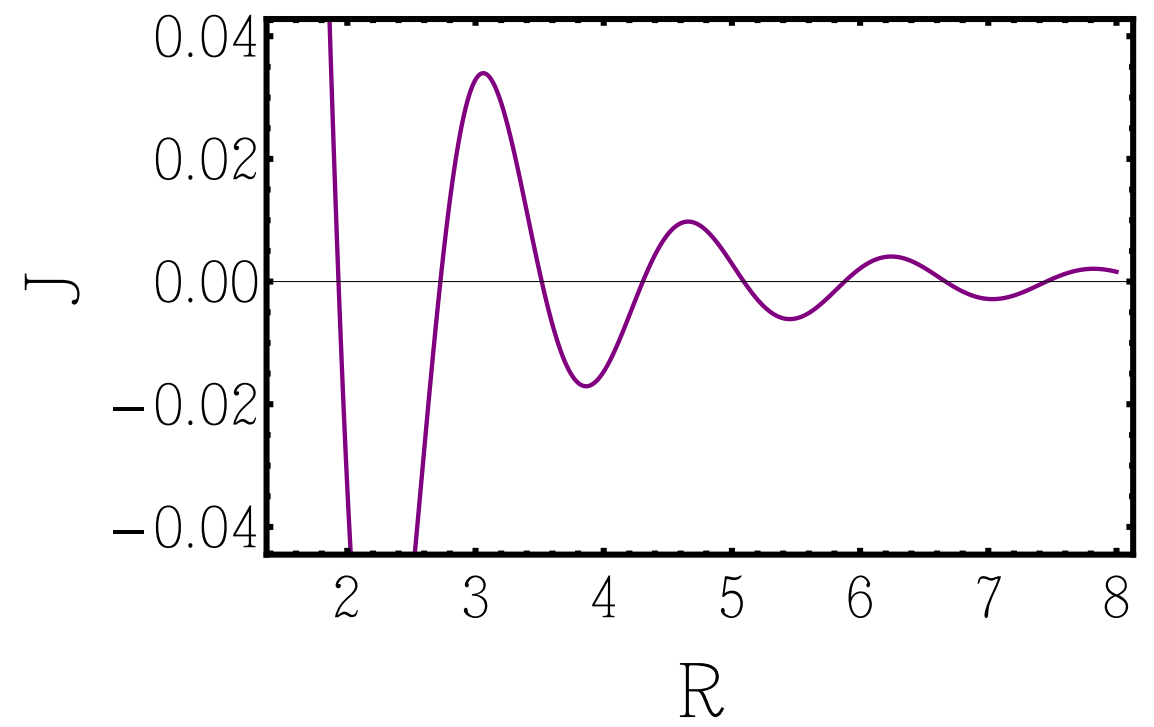

Figure 2.4: Oscillatory behavior of $J$ in unit of $m \pi^{-3} \lambda^{2}$ as a function of $R$ in the units of $\AA$ for given value of $k_{F}=1.2(1 / \AA)$ in a three-dimensional electron gas.

The integral can be evaluated by a straightforward contour integration [24] to yield the result

$$
G\left(\vec{r}_{1}, \vec{r}_{2}, E\right)=\frac{-m}{2 \pi r \hbar^{2}}\left(\begin{array}{cc}
e^{i \alpha(E) r} & 0 \\
0 & e^{i \alpha(E) r}
\end{array}\right),
$$

where $r \equiv\left|\vec{r}_{1}-\vec{r}_{2}\right|$ and

$$
\alpha(x)=\left\{\begin{array}{l}
\left(2 m \hbar^{-2} x\right)^{1 / 2} \text { if } x>0, \\
i\left(2 m \hbar^{-2}|x|\right)^{1 / 2} \text { if } x<0 .
\end{array}\right.
$$

Plugging these into Eq. (2.25) and performing the energy integrations, the results for the magnetic interaction terms are given by

$$
J=2 I\left(k_{F} R\right)
$$


where $E_{F}$ is the Fermi energy, $k_{F}=\left(2 m \hbar^{-2} E_{F}\right)^{1 / 2}$ being the Fermi momentum and

$$
I(x)=-\frac{\lambda^{2} m}{(4 \pi)^{3} R^{4}} \times[\sin (2 x)-2 x \cos (2 x)]
$$

We illustrate the oscillatory behavior of $J$ in Fig. (2.4) for typical parameters.

\subsection{One-Dimensional Spin-Polarized Electron Gas}

Although Eq. (2.19) is a known formula and has been widely used in the literature $[5,6,7,29]$, working through its derivation still holds value. A pedagogical derivation of this equation, which is given in the Appendix, provides some important information about limitation of using this equation to get the indirect exchange interaction between two localized magnetic moments, and can be helpful for both experts and beginners in this research field. A very essential point that can be extracted is to get the result as Eq. (2.19), the order of integration between $E$ and $\vec{k}$, immediately after Eq. (2.9), has been changed. If the Fubini's condition [20, 21] is not satisfied, changing the order of integration in a double-intergral can lead to a different result. For the case of one-dimensional electron gas, one can immediately find that Fubini's condition is not satisfied.

For the case of one-dimensional spin-polarized electron gas the unperturbed eigen-

states and energy bands are defined as $|\vec{k} \sigma\rangle=\frac{1}{\sqrt{L}} e^{i \vec{k} \cdot \vec{r}}|\sigma\rangle$ and $\varepsilon_{\vec{k} \sigma}=\frac{\hbar^{2} k^{2}}{2 m} \mp \Delta$, respectively, where we have assumed $+(-)$ sign for spin-down (-up) states. To find the elements of Green's function matrix, one can use Eq. (2.20) and find the result 


$$
G\left(\vec{r}_{1}, \vec{r}_{2}, E\right)=-\frac{i m}{\hbar^{2}}\left(\begin{array}{cc}
\frac{e^{i \alpha(E+\Delta) r}}{\alpha(E+\Delta)} & 0 \\
0 & \frac{e^{i \alpha(E-\Delta) r}}{\alpha(E-\Delta)}
\end{array}\right)
$$

where

$$
\alpha(x)=\left\{\begin{array}{l}
\left(2 m \hbar^{-2} x\right)^{1 / 2} \text { if } x>0, \\
i\left(2 m \hbar^{-2}|x|\right)^{1 / 2} \text { if } x<0 .
\end{array}\right.
$$

For this case, $G_{\sigma \sigma^{\prime}}(\vec{R}, 0, E)=G_{\sigma \sigma^{\prime}}(0, \vec{R}, E)$. The last result could be expected due to the presence of inversion symmetry. If we set $\Delta=0$, we can easily find the results for the Green's function matrices for the case of standard one-dimensional electron gas. Writing the interaction in the form of $E=E_{0}+E(\vec{R})$, where $E_{0}$ is a constant shift to the energy of the system and $E(\vec{R})$ is the distance-dependent part of the interaction, and using the first-order perturbation theory, one can easily find

$$
\begin{aligned}
E_{0} & =\sum_{\vec{k} \nu}^{o c c}\left\langle\vec{k} \nu\left|\hat{V}_{1}+\hat{V}_{2}\right| \vec{k} \nu\right\rangle, \\
& =-\frac{\lambda \hbar}{2 \pi}\left(S_{1 z}+S_{2 z}\right)\left(k_{F \uparrow}-k_{F \downarrow}\right) .
\end{aligned}
$$

Choosing the $z$-direction as the quantization axis leads to this non-zero $E_{0}$ for spinpolarized electron gas. For the case of standard one-dimensional electron gas, $k_{F \uparrow}=$ $k_{F \downarrow}$ and $E_{0}=0$.

Using similar methods, and defining $\delta=\sqrt{2 m \hbar^{-2} \Delta}$, we found the $\vec{R}$-dependent part of the interaction between two localized magnetic moments in the one-dimensional 
spin-polarized electron gas has the form of $E(\vec{R})=J \vec{S}_{1} \cdot \vec{S}_{2}+\vec{S}_{1} \cdot \stackrel{\leftrightarrow}{\Gamma} \cdot \vec{S}_{2}$, where

$$
J=\frac{\lambda^{2} m}{4 \pi}\left\{\operatorname{si}\left(2 k_{F \uparrow} R\right)+\operatorname{si}\left(2 k_{F \downarrow} R\right)\right\}
$$

and the tensor interaction has the form of:

$$
\stackrel{\leftrightarrow}{\Gamma}=\left(\begin{array}{ccc}
P & 0 & 0 \\
0 & P & 0 \\
0 & 0 & 0
\end{array}\right)
$$

where

$$
\begin{aligned}
P & =\frac{\lambda^{2} m}{2 \pi}\left(-\int_{0}^{\sqrt{2} \delta} \frac{e^{-\sqrt{2 \delta^{2}-k^{2}} R} \cos [k R]}{\sqrt{2 \delta^{2}-k^{2}}} d k\right. \\
& +\int_{\sqrt{2} \delta}^{k_{F \uparrow}} \frac{\sin \left[\sqrt{k^{2}-2 \delta^{2}} R\right] \cos [k R]}{\sqrt{k^{2}-2 \delta^{2}}} d k \\
& \left.+\int_{0}^{k_{F \downarrow}} \frac{\sin \left[\sqrt{k^{2}+2 \delta^{2}} R\right] \cos [k R]}{\sqrt{k^{2}+2 \delta^{2}}} d k-g\right),
\end{aligned}
$$

with

$$
g= \begin{cases}J & \text { if } \delta \neq 0 \\ J+\frac{\pi}{2} & \text { if } \delta=0\end{cases}
$$

As it can be seen, we found $\vec{D}=0$. This result could be physically expected, based on the lack of explicit spin-orbit coupling and the presence of inversion symmetry in the system. Note that for an unpolarized electron gas $\Delta=0$, so $\delta=0$ which leads to $k_{F \uparrow}=k_{F \downarrow}$, and finally, above expressions lead to $P=0$ and the well-known $J$ for the 

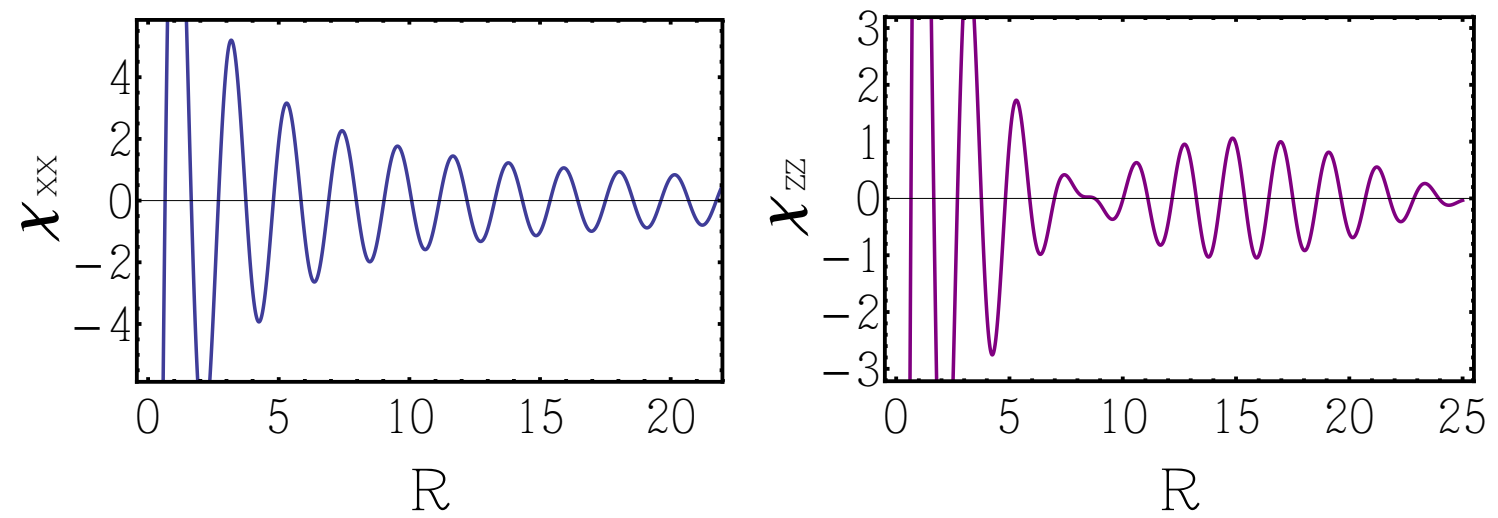

Figure 2.5: Oscillatory behaviors of $\chi_{z z}$ (right) and $\chi_{x x}$ (left) in units of $10^{-2} m \pi^{-1}$ as a function of $R$ in the units of $\AA$ for given values of $k_{F \uparrow}=1.574$ and $k_{F \downarrow}=1.388$ $1 / \AA$. The beat-pattern oscillatory behavior of $\chi_{z z}$ can be easily understood based on two different Fermi momenta, $k_{F \uparrow}$ and $k_{F \downarrow}$, in the system.

case of one-dimensional electron gas [5, 22, 23, 24]. Adding all of the energy terms, it is convenient to find an anisotropic Heisenberg form for this interaction,

$$
E(\vec{R})=\lambda^{2} \chi_{x x}\left(S_{1 x} S_{2 x}+S_{1 x} S_{2 x}\right)+\lambda^{2} \chi_{z z} S_{1 z} S_{2 z}
$$

with $\chi_{x x}=\left(P+J^{1 D}\right) / \lambda^{2}$ and $\chi_{z z}=J^{1 D} / \lambda^{2}$. Oscillatory behaviors of $\chi_{x x}$ and $\chi_{z z}$ as a function of $R$, have been studied in the Fig. (2.5).

To study the long-range behavior of $\chi_{z z}$, one can write the sine integral in terms of auxiliary functions, $\operatorname{Si}(x) \simeq \frac{\pi}{2}-f(x) \cos (x)-g(x) \sin (x)$, then use asymptotic series,

$$
\begin{aligned}
& f(x) \simeq \frac{1}{x}\left(1-\frac{2 !}{x^{2}}+\frac{4 !}{x^{4}}-\frac{6 !}{x^{6}}+\ldots\right) \\
& g(x) \simeq \frac{1}{x^{2}}\left(1-\frac{3 !}{x^{2}}+\frac{5 !}{x^{4}}-\frac{7 !}{x^{6}}+\ldots\right),
\end{aligned}
$$


so we will get $\operatorname{si}(x) \simeq-\cos (x) / x$ which leads to the result

$$
\chi_{z z} \simeq-\frac{m}{4 \pi}\left(\frac{\cos \left(2 k_{F \uparrow} R\right)}{2 k_{F \uparrow} R}+\frac{\cos \left(2 k_{F \downarrow} R\right)}{2 k_{F \downarrow} R}\right)
$$

for large distances, $R \gg k_{F \uparrow}^{-1}$ and $k_{F \downarrow}^{-1}$. This result is compatible with the well-known long-range behavior of RKKY interaction in $n$-dimension, viz.,

$$
\chi(\vec{R}) \propto \frac{\cos \left(2 k_{F} R-\frac{(n-1) \pi}{2}\right)}{\left(2 k_{F} R\right)^{n}} .
$$

The last result is different from what Sobota et al. [27] reported and provides right answer for the long-distance behavior of RKKY interaction in $n$-dimension.

\subsection{Two-Dimensional Spin-Polarized Electron Gas}

The Green's function for the spin polarized electron gas in 2D is given by

$$
G_{\sigma \sigma^{\prime}}\left(\vec{r}, \vec{r}^{\prime}, E\right)=\frac{\delta_{\sigma \sigma^{\prime}}}{(2 \pi)^{2}} \int \frac{e^{i \vec{k} \cdot\left(\vec{r}-\vec{r}^{\prime}\right)}}{E+i \eta-\varepsilon_{\vec{k} \sigma}} d^{2} k
$$

This a standard integration, which can be evaluated by using the Jacobi-Anger expansion of the exponential term in terms of the Bessel's functions and by performing the angular integration. [25, 26] The result is [28, 29]

$$
G_{\sigma \sigma}\left(\vec{r}, \vec{r}^{\prime}, E\right)=-\frac{m}{\pi \hbar^{2}} K_{0}\left[-i \frac{\sqrt{2 m}}{\hbar}\left|\vec{r}-\vec{r}^{\prime}\right| \alpha(E \pm \Delta)\right]
$$


where $+(-)$ is for $\sigma=\uparrow(\downarrow), K_{0}$ is the modified Bessel function of the second kind, and

$$
\alpha(x)= \begin{cases}\sqrt{x} & \text { if } x>0 \\ i \sqrt{|x|} & \text { if } x<0\end{cases}
$$

To find the imaginary and the real parts of the modified Bessel function, it is convenient to use the equality $K_{\nu}(x)=2^{-1} \pi i^{\nu+1} H_{\nu}^{1}(i x)$, which is valid for $-\pi<\arg (x) \leq$ $\pi / 2$. The Hankel function of the first kind is written in terms of the Bessel and Neumann functions as $H_{\nu}{ }^{1}(x)=J_{\nu}(x)+i Y_{\nu}(x)$. The expansion coefficients for the Green's function, Eq. (2.21), are then $g_{0}=2^{-1}\left(G_{\uparrow \uparrow}\left(\vec{r}, \vec{r}^{\prime}, E\right)+G_{\downarrow \downarrow}\left(\vec{r}, \vec{r}^{\prime}, E\right)\right)$ and $g_{z}=2^{-1}\left(G_{\uparrow \uparrow}\left(\vec{r}, \vec{r}^{\prime}, E\right)-G_{\downarrow \downarrow}\left(\vec{r}, \vec{r}^{\prime}, E\right)\right)$. Plugging these into Eq. (2.24), we find the results $[28,29]$

$$
\begin{aligned}
& J_{1}=\frac{\lambda^{2} m^{2}}{8 \pi \hbar^{2}}\left\{-\frac{2}{\pi} \int_{-\Delta}^{\Delta} \operatorname{Re}\left[K_{0}(\kappa R)\right] J_{0}\left(k_{+} R\right) d E\right. \\
& \left.+\int_{\Delta}^{E_{F}}\left[J_{0}\left(k_{-} R\right) Y_{0}\left(k_{+} R\right)+Y_{0}\left(k_{-} R\right) J_{0}\left(k_{+} R\right)\right] d E\right\} \\
& J_{2}=\frac{\lambda^{2} m}{16 \pi R^{2}}\left[I^{\prime}\left(k_{F-} R\right)+I^{\prime}\left(k_{F+} R\right)\right]-J_{1},
\end{aligned}
$$

where $I^{\prime}(x)=x^{2}\left[J_{0}(x) Y_{0}(x)+J_{1}(x) Y_{1}(x)\right]$.

In this case, similar to the case of 3D spin-polarized electron gas, the oscillatory behaviors of $J_{1}$ and $J_{2}$ show beat-pattern, caused by the two different Fermi momenta for the two spin channels. We got the same results using another method, method of summation over $\vec{k}$, which can be found in the Appendix. 


\subsection{Three-Dimensional Spin-Polarized Electron Gas}

Our starting point is the electron band structure

$$
\varepsilon_{\vec{k} \sigma}=\frac{\hbar^{2} k^{2}}{2 m} \mp \Delta
$$

where $-(+)$ sign is for spin up (down) states, so that $2 \Delta$ is the band splitting between the up- and down-spin states. The corresponding plane-wave eigenstates are

$$
|\vec{k} \sigma\rangle=\frac{1}{\sqrt{\Omega}} e^{i \vec{k} \cdot \vec{r}}|\sigma\rangle
$$

where the $\Omega$ is the volume of the box for normalization. The key quantity to evaluate is the Green's function, which, using Eqs. (2.20), (2.59), and (2.60) and converting the summation into integration in the momentum space, is written as

$$
G_{\sigma \sigma^{\prime}}\left(\vec{r}, \vec{r}^{\prime}, E\right)=\frac{\delta_{\sigma \sigma^{\prime}}}{(2 \pi)^{3}} \int \frac{e^{i \vec{k} \cdot\left(\vec{r}-\vec{r}^{\prime}\right)}}{E+i \eta-\varepsilon_{\vec{k} \sigma}} d^{3} k .
$$

The integral can be evaluated by a straightforward contour integration [28, 29] to yield the result

$$
\begin{aligned}
& G\left(\vec{r}_{1}, \vec{r}_{2}, E\right)=\left(\begin{array}{cc}
g_{\uparrow} & 0 \\
0 & g_{\downarrow}
\end{array}\right) \\
& =\frac{-m}{2 \pi r \hbar^{2}}\left(\begin{array}{cc}
e^{i \alpha(E+\Delta) r} & 0 \\
0 & e^{i \alpha(E-\Delta) r}
\end{array}\right),
\end{aligned}
$$


where $r \equiv\left|\vec{r}_{1}-\vec{r}_{2}\right|$ and

$$
\alpha(x)=\left\{\begin{array}{l}
\left(2 m \hbar^{-2} x\right)^{1 / 2} \text { if } x>0, \\
i\left(2 m \hbar^{-2}|x|\right)^{1 / 2} \text { if } x<0 .
\end{array}\right.
$$

The coefficients $g_{0}$ and $g_{z}$ in Eq. (2.21) are then $g_{0}=\left(g_{\uparrow}+g_{\downarrow}\right) / 2$ and $g_{z}=\left(g_{\uparrow}-g_{\downarrow}\right) / 2$, which are complex numbers.

Plugging these into Eq. (2.24) and performing the energy integrations, we find that the imaginary part vanishes, as it must, and the results for the magnetic interaction terms are given by

$$
\begin{aligned}
J_{1}=\frac{-\lambda^{2} m^{2}}{8 \pi^{3} \hbar^{2} R^{2}} \times\left(\int_{\Delta}^{E_{F}} \sin \left[k_{+}(E) R+k_{-}(E) R\right)\right] d E \\
\left.\quad+\int_{-\Delta}^{\Delta} \exp [-\kappa(E) R] \times \sin \left[k_{+}(E) R\right] d E\right), \\
J_{2}=I\left(k_{F-} R\right)+I\left(k_{F+} R\right)-J_{1},
\end{aligned}
$$

where $E_{F}$ is the Fermi energy, $k_{ \pm}(E)=\left[2 m \hbar^{-2}(E \pm \Delta)\right]^{1 / 2}$ is the momentum for the spin up (down) state, $k_{F \pm} \equiv k_{ \pm}\left(E_{F}\right)$ is the corresponding Fermi momentum for spin up (down) electrons, $\kappa(E)=\left[2 m \hbar^{-2}(\Delta-E)\right]^{1 / 2}$, and

$$
I(x)=-\frac{\lambda^{2} m}{(4 \pi)^{3} R^{4}} \times[\sin (2 x)-2 x \cos (2 x)] .
$$

The integral $I(x)$ is familiar from the theory of the spin-unpolarized electron gas, in which case, the RKKY interaction is, simply,

$$
J_{1}=2 I\left(k_{F} R\right)
$$


$k_{F}=\left(2 m \hbar^{-2} E_{F}\right)^{1 / 2}$ being the Fermi momentum. The results agree with our earlier work, where we had used a different method [24].
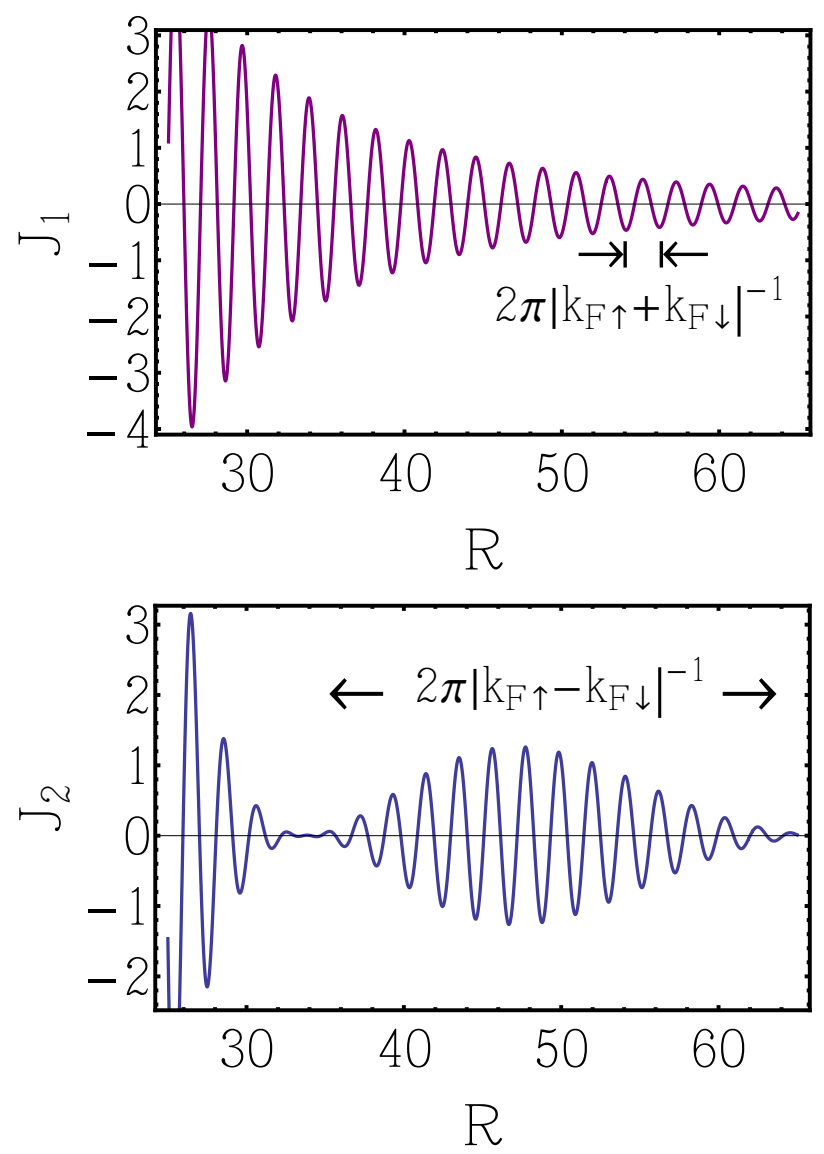

Figure 2.6: Oscillatory behavior of $J_{1}$ and $J_{2}$, in the units of $10^{-5} m \lambda^{2} \pi^{-3}$ as a function of $R$ in the units of $\AA$ for the case of iron.

Note that if there is no spin polarization $(\Delta=0)$, then Eq. (2.64) immediately reduces to the expression Eq. (2.66) for the spin-unpolarized gas and $J_{2}=0$. The computed results for $J_{1}$ and $J_{2}$, for the case of iron, are shown in Fig. 2.6, which show the oscillatory behavior characteristic of the inverse momentum $\left(k_{F+}+k_{F-}\right)^{-1}$, and the beat pattern for $J_{2}$ is characteristic of the inverse difference $\left(k_{F+}-k_{F-}\right)^{-1}$, respectively. Band calculations for iron [31] lead to the $E_{F} \approx 8 \mathrm{eV}$ and $2 \Delta \approx 2 \mathrm{eV}$. 
One can use these values to find the Fermi momenta, $k_{F \uparrow} \simeq 1.574$ and $k_{F \downarrow} \simeq 1.3881 / \AA$ . For long distances, we predict the oscillation periods to be: $2 \pi\left|k_{F \uparrow}+k_{F \downarrow}\right|^{-1} \simeq 2.12$ $\AA$ and for the beat pattern behavior $2 \pi\left|k_{F \uparrow}-k_{F \downarrow}\right|^{-1} \simeq 33.75 \AA$. Fig. 2.6 shows a very good match between the computed results and predictions.

Discussions - An interesting situation occurs if $J_{1}+J_{2}=0$, which can happen for certain distances. In this case, the net interaction, Eq. (2.23), assumes the form: $E(\vec{R})=J_{1}\left(S_{1 x} S_{2 x}+S_{1 y} S_{2 y}\right)$, which would clearly align the spins in the $x y$-plane, i. e., normal to the spin polarization axis. In general, depending on the relative strengths of $J_{1}(\vec{R})$ and $J_{2}(\vec{R})$, the net spin interaction could align the two spins in different directions, leading to the possibility for unusual spin textures.

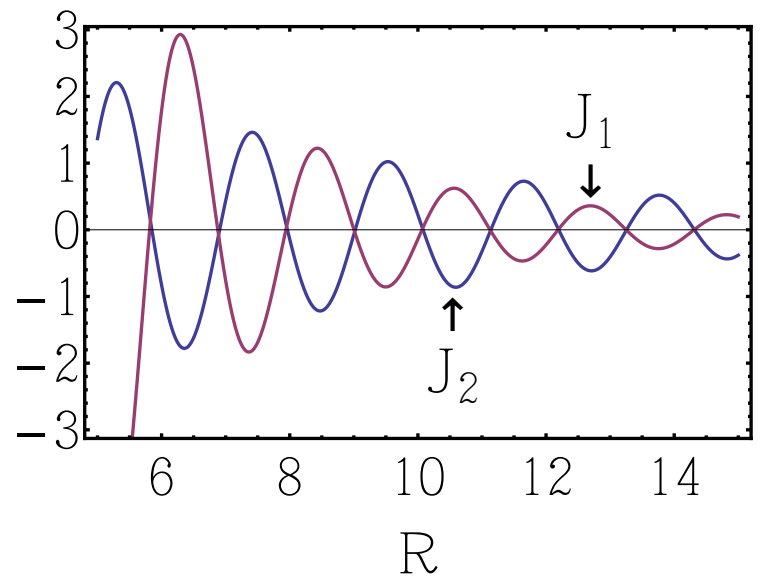

Figure 2.7: Oscillatory behavior of $J_{1}$ and $J_{2}$, in the units of $10^{-3} m \lambda^{2} \pi^{-3}$ as a function of $R$ in the units of $\AA$ for the case of iron.

Note that apart from the position dependent interaction $E(\vec{R})$, there is a constant energy shift

$$
E_{0}=\frac{-\hbar \lambda}{12 \pi^{2}}\left(S_{1 z}+S_{2 z}\right)\left(k_{F \uparrow}^{3}-k_{F \downarrow}^{3}\right)
$$

a new term not present in the standard, non-spin-polarized electron gas, and a term 
that tends to align the embedded spins $\vec{S}_{1}$ and $\vec{S}_{2}$ along the $\hat{z}$ axis. This expression, obtained by using the first-order perturbation theory for the perturbing potentials, Eqs. (2.17) and (2.18), depends on the strength of the polarization of the electron gas and would dominate for strong spin polarization. In turn, for weak spin polarization $\left(\delta k_{F} \equiv k_{F+}-k_{F-} \ll m \lambda \bar{k}_{F}^{2} /\left(R \bar{k}_{F}\right)^{4}\right.$, where $\left.\bar{k}_{F} \equiv\left(k_{F+}-k_{F-}\right) / 2\right)$, this term is negligible and the $J_{1}$ and $J_{2}$ interactions dominate.

Another point to note is that the essential ingredient for the presence of the DM interactions is the broken symmetry (time reversal or inversion or both). In the original DM work[17], the spin-orbit coupling (SOC) provided the mechanism for the magnetic interaction. The interaction between the magnetic moments of two atoms occurred via an intermediate atom and involved the spin-orbit coupled excited states on the two atoms. In this case, as originally showed by DM, $J \sim \xi^{0}$, $\vec{D} \sim \xi$, and $\stackrel{\leftrightarrow}{\Gamma} \sim \xi^{2}$, where $\xi$ is the spin-orbit coupling strength $(\xi \vec{L} \cdot \vec{S})$, so that $|\stackrel{\leftrightarrow}{\Gamma}| \ll|\vec{D}| \ll|J|, \xi$ being a small parameter, and it is then customary to ignore the tensor DM interaction $\stackrel{\leftrightarrow}{\Gamma}$. In the present case, the broken time-reversal symmetry without the involvement of any SOC leads to the DM interaction, so that it is entirely a different mechanism, and further that the strengths of all terms are comparable, being proportional to $\lambda^{2}$. Thus, in a solid, if a spin-polarized electron gas is present in addition to magnetic moments on atoms with SOC, both effects must be considered separately and the dominant effect for the DM interactions might as well come from the spin polarization of the electron gas, of the type studied in this chapter. We got the same results using another method, method of summation over $\vec{k}$, which can be found in the Appendix. 


\subsection{Summary}

In this chapter, we obtained the magnetic interactions between two localized moments, embedded in the spin polarized electron gas in one, two and three dimensions, extending the standard results for the spin-unpolarized electron gas, which leads to the well known RKKY interaction. The spin-polarization leads to an anisotropic Heisenberg type of interaction, of the form $J_{1} \vec{S}_{1} \cdot \vec{S}_{2}+J_{2} S_{1 z} S_{2 z}$. Both terms $J_{1}$ and $J_{2}$ show oscillatory behavior as a function of distance between the two magnetic moments with the period of the oscillations determined by $\bar{k}_{F} R, \bar{k}_{F}$ being the average Fermi momentum of the two spin channels and, in addition, $J_{2}$ shows a beating pattern determined by the momentum difference $k_{F+}-k_{F-}$. This is the simplest system with broken symmetry and serves to illustrate the origin of the magnetic interactions in the solid that go beyond the standard RKKY $\vec{S}_{1} \cdot \vec{S}_{2}$ type interaction. It is worthwhile to mention that, disregarding the coefficients and the decay-factor $R^{-n}$, the long-distance behaviors of $J$ for the cases of one- and three-dimensional spin-polarized electron gas show

a similar pattern, viz., $J \propto \cos \left(k_{F \uparrow} R+k_{F \downarrow} R\right) \times \cos \left(k_{F \uparrow} R-k_{F \downarrow} R\right)$, while for the case of two-dimensional spin-polarized electron gas, $\sin \left(k_{F \uparrow} R+k_{F \downarrow} R\right) \times \cos \left(k_{F \uparrow} R-k_{F \downarrow} R\right)$ form of oscillatory behavior is expected. 


\section{Chapter 3}

\section{RKKY and DM Interactions in a Two-dimensional Electron Gas with Rashba and Dresselhaus Spin-Orbit Couplings}

The first efforts to study and generalize this interaction in a system with broken inversion symmetry and in the presence of SOC were done by Dzyaloshinski and Moriya $[16,17](\mathrm{DM})$, who showed this situation leads to additional vector and tensor

form of interactions, $\vec{D} \cdot \vec{S}_{1} \times \vec{S}_{2}$ and $\vec{S}_{1} \cdot \stackrel{\leftrightarrow}{\Gamma} \cdot \vec{S}_{2}$, and are known as vector and tensor DM interactions. RKKY interaction yields a ferromagnetic or antiferromagnetic coupling of localized moments, while vector DM interaction would like to produce a nonlinear coupling of them. It has been shown[24, 28, 29] that in the presence of inversion symmetry vector DM interaction vanishes, the tensor DM can be potentially present. RKKY interaction in a spin-polarized electron gas, where time-reversal symmetry is broken and inversion symmetry is present, is an example. In the last scenario, RKKY 
and tensor DM interaction can be combined and obtain an anisotropic Heisenberg from of interaction.

Strength of vector and tensor DM interactions can be manipulate in the tunable SOC host materials $[32,33,34,35,36,37]$. This is a great deal of interest and can be really important in spintronics, where the spin degrees of freedom of electrons will be manipulate to result a preferred outcome. Well-know Rashba[15] and less-studied Dresselhaus[38] SOC are two examples where can be tuned in some materials by changing external electric field and changing the well-width of the system, respectively.

Based on the importance of the problem, interaction between two localized moments in a non-magnetic electron gas with Rashba SOC has been widely studied in the literature. Using the Green's function approach, which will be used in the present work, Imamura et al.[5] found a promising analytical results for long-distance behavior of RKKY and DM interactions in both one- and two-dimensions, valid for large Fermi-energy limits, viz., $E_{F} \gg\left(m \alpha^{2}\right) /\left(2 \hbar^{2}\right)$. In addition to Lyu et al. [39] who studied the exact same problem numerically, Mross and Johannesson[40] have found very similar analytical results for the same limits. In the same spirit, Huang and co-authors[41] have investigated similar problem and numerically studied the crucial role of topology of the Fermi surface on both DM and RKKY interactions. Chesi and Loss[8] have included the Dresselhaus SOC to the problems, and carefully studied the behavior of RKKY and DM interactions for certain limits in a disordered twodimensional electron gas with both Rashba and Dresselhaus spin-orbit couplings. All of the previously mentioned works have been done for a non-magnetic host material, where there is not any spin-polarization in the system. Kim et al.[42] brought spin- 
polarization to the picture by studying the chirality made by the Rashba SOC in a magnetic material. With a different approach, similar problem has been numerically investigated by Kundu and Zhang[43]. In that work, the authors studied the effect of spin-polarization on the DM interaction, and have carefully analyzed the importance of each term.

\subsection{Formalism}

We consider a 2D spin-polarized electron gas in the presence of Rashba and Dresselhaus spin-orbit interaction, which is relevant, for example, for a magnetic thin film with bulk and surface broken inversion symmetry. The broken bulk inversion symmetry produces the Dresselhaus term, while the surface asymmetry produces the Rashba term, and recently it has been possible[33] to tune the relative magnitudes of the two terms experimentally by adjusting the external electric field and the thickness of the 2D structure. The system is described by the Hamiltonian

$$
\mathcal{H}=\frac{\hbar^{2} k^{2}}{2 m} \sigma_{0}+\alpha\left(k_{y} \sigma_{x}-k_{x} \sigma_{y}\right)+\beta\left(k_{x} \sigma_{x}-k_{y} \sigma_{y}\right)+\Delta \sigma_{z}
$$

where $\alpha$ and $\beta$ are, respectively, the strength of the Rashba and the Dresselhaus term, $\sigma_{i}$ are the Pauli matrices, $\sigma_{0}$ is the unit $2 \times 2$ matrix, $\vec{k}$ is the electron momentum, and $\Delta$ describes the Zeeman spin splitting of the electron states.

General Formalism - We are interested in the magnetic interaction between two

localized moments, $\vec{S}_{1}$ and $\vec{S}_{2}$, embedded in the host electrons and which interact 
with the host electrons via the contact interaction

$$
V_{i}(\vec{r})=-\lambda \delta\left(\vec{r}-\vec{R}_{i}\right) \vec{S}_{i} \cdot \vec{s}
$$

where $\vec{R}_{1}=0$ and $\vec{R}_{2}=\vec{R}$ are the positions of the two moments and $\vec{s}=\hbar \vec{\sigma} / 2$.

The magnetic interaction is conveniently expressed as integrals over the expansion coefficients of the Green's functions in terms of the Dirac matrices. Below we derive these results, which are true for any general case irrespective of the dimensionality of the system.

The interaction energy $E(\vec{R})$ between the two moments is given by the well-known expression[24, 29]

$$
\begin{aligned}
E(\vec{R})= & \frac{-\lambda^{2}}{\pi} \operatorname{Im} \int_{-\infty}^{E_{F}} \operatorname{Tr}\left[G(0, \vec{R}, E) \vec{S}_{2} \cdot \vec{s}\right. \\
& \left.G(\vec{R}, 0, E) \vec{S}_{1} \cdot \vec{s}\right] d E
\end{aligned}
$$

where $G(E)=(E+i \eta-\mathcal{H})^{-1}$ with $\eta \rightarrow 0^{+}$, is the retarded GF, and $E_{F}$ is the Fermi energy. The Zeeman term in Eq. (3.1) introduces a net spin polarization in the electron gas for $\Delta \neq 0$, leading to a constant shift in energy given by

$$
E_{0}=-\frac{\lambda \hbar}{2}\left(S_{1 z}+S_{2 z}\right)\left(n_{\uparrow}-n_{\downarrow}\right)
$$

The spin polarization $n_{\uparrow}-n_{\downarrow}$ depends on the system and for the 2DEG under study here, it is easily shown to be $n_{\uparrow}-n_{\downarrow}=4 \pi(2 m)^{1 / 2} \hbar^{-1} \times\left[\left(E_{F}+\Delta\right)^{1 / 2}-\left(E_{F}-\Delta\right)^{1 / 2}\right]$, e. g., from the wave functions given later in Eq. (3.13). Including this energy shift, 
the total energy becomes

$$
E=E_{0}+E(\vec{R})+O\left(\lambda^{3}\right)
$$

The GFs in Eq. (3.3) are $2 \times 2$ matrices in the spin space with the matrix elements given by

$$
G_{\sigma_{1} \sigma_{2}}\left(\vec{r}_{1}, \vec{r}_{2}, E\right)=\sum_{\vec{k} \nu}^{\infty} \frac{\psi_{\vec{k} \nu}\left(\vec{r}_{1}, \sigma_{1}\right) \psi_{\vec{k} \nu}^{*}\left(\vec{r}_{2}, \sigma_{2}\right)}{E+i \eta-\varepsilon_{\vec{k} \nu}},
$$

where $G_{\sigma_{1} \sigma_{2}}\left(\vec{r}_{1}, \vec{r}_{2}, E\right) \equiv\left\langle\vec{r}_{1} \sigma_{1}|\hat{G}(E)| \vec{r}_{2} \sigma_{2}\right\rangle, \psi_{\vec{k} \nu}(\vec{r}, \sigma)=\langle\vec{r} \sigma \mid \vec{k} \nu\rangle$, and $\vec{k} \nu$ labels the eigenstates of the system.

It is convenient to write the GFs in terms of the Pauli matrices and express the interaction energy Eq. (3.3) in terms of the trace of products of the Pauli matrices. We thus have

$$
\begin{aligned}
& G(\vec{R}, 0, E)=g_{0} \sigma_{0}+\sum_{i=1}^{3} g_{i} \sigma_{i}, \\
& G(0, \vec{R}, E)=g_{0}^{\prime} \sigma_{0}+\sum_{i=1}^{3} g_{i}^{\prime} \sigma_{i},
\end{aligned}
$$

where the two GFs are different if we don't have inversion symmetry, as is true for the present case. Using the trace identities

$$
\begin{aligned}
& \operatorname{Tr}\left(\sigma_{i} \sigma_{j}\right)=2 \delta_{i j}, \\
& \operatorname{Tr}\left(\sigma_{i} \sigma_{j} \sigma_{k}\right)=2 i \varepsilon_{i j k}, \\
& \operatorname{Tr}\left(\sigma_{i} \sigma_{j} \sigma_{k} \sigma_{l}\right)=2\left(\delta_{i j} \delta_{k l}-\delta_{i k} \delta_{j l}+\delta_{i l} \delta_{j k}\right),
\end{aligned}
$$

where $\delta_{i j}$ are the Kronecker deltas and $\varepsilon_{i j k}$ are the Levi-Civita symbols, it is straight- 
forward to evaluate the interaction energy (3.3), which yields

$$
E(\vec{R})=J \vec{S}_{1} \cdot \vec{S}_{2}+\vec{D} \cdot \vec{S}_{1} \times \vec{S}_{2}+\vec{S}_{1} \cdot \stackrel{\leftrightarrow}{\Gamma} \cdot \vec{S}_{2}
$$

Defining vectors $\vec{g}=\sum_{i=1}^{3} g_{i} \hat{i}$ and $\vec{g}^{\prime}=\sum_{i=1}^{3} g_{i}^{\prime} \hat{i}$, the expressions for the RKKY and the Dzyaloshinskii-Moriya interaction constants are written as

$$
\begin{aligned}
& J=\frac{-\lambda^{2} \hbar^{2}}{2 \pi} \int_{-\infty}^{E_{F}} \operatorname{Im}\left(g_{0}^{\prime} g_{0}-\vec{g}^{\prime} \cdot \vec{g}\right) d E \\
& \vec{D}=\frac{-\lambda^{2} \hbar^{2}}{2 \pi} \int_{-\infty}^{E_{F}} \operatorname{Re}\left(g_{0}^{\prime} \vec{g}-g_{0} \vec{g}^{\prime}\right) d E \\
& \stackrel{\leftrightarrow}{\Gamma}=\frac{-\lambda^{2} \hbar^{2}}{2 \pi} \int_{-\infty}^{E_{F}} \operatorname{Im}\left(\vec{g} \vec{g}^{\prime}+\vec{g}^{\prime} \vec{g}\right) d E .
\end{aligned}
$$

The problem thus boils down to the calculation of the GF coefficients $\left(g_{0}, \vec{g}, g_{0}^{\prime}, \vec{g}^{\prime}\right)$ for the given Hamiltonian.

These equations represent a central result of the paper. They are valid quite generally, i. e., for any system with a $2 \times 2$ spin Hamiltonian matrix, irrespective of the dimensionality of the system.

Note that if the inversion symmetry is present, e.g., when the Rashba and Dresselhaus terms $\alpha$ and $\beta$ are zero, we have $G(\vec{R}, 0, E)=G(0, \vec{R}, E)$, so that $g_{0}=g_{0}^{\prime}$ and $\vec{g}=\vec{g}^{\prime}$. In this case, Eq. (3.10) immediately leads to the well-known result $\vec{D}=0$, i. e., the absence of the DM interaction for a system with inversion symmetry.

Simplified expressions for the 2DEG - For the 2DEG described by the Hamiltonian (3.1), the calculation becomes a bit simplified due to the fact that

$$
g_{0}^{\prime}=g_{0}, \quad g_{1}^{\prime}=-g_{1}, \quad g_{2}^{\prime}=-g_{2}, \text { and } \quad g_{3}^{\prime}=g_{3}
$$


This can be easily proved. To show this, we find the eigenvalues of Hamiltonian (3.1), which read

$$
\varepsilon_{\nu}(\vec{k})=\frac{\hbar^{2} k^{2}}{2 m}+(-1)^{\nu}\left[\Delta^{2}+\left(\alpha^{2}+\beta^{2}+2 \alpha \beta \sin 2 \theta\right) k^{2}\right]^{1 / 2}
$$

where $\nu=1,2$ is the band index and $\theta$ is the polar angle in $k$-space, $\theta=\tan ^{-1}\left(k_{y} / k_{x}\right)$, and the corresponding eigenfunctions are

$$
\begin{aligned}
|\vec{k} \nu\rangle & =\left(\begin{array}{c}
a_{\vec{k} \nu}^{\uparrow} \\
a_{\vec{k} \nu}^{\downarrow}
\end{array}\right), \\
a_{\vec{k} \nu}^{\uparrow} & =\Lambda_{\vec{k}, \nu}\left(1+\left|\Lambda_{\vec{k}, \nu}\right|^{2}\right)^{-1 / 2}, \\
a_{\vec{k} \nu}^{\downarrow} & =\left(1+\left|\Lambda_{\vec{k}, \nu}\right|^{2}\right)^{-1 / 2}, \\
\Lambda_{\vec{k}, \nu} & =\frac{i\left(\Delta+(-1)^{\nu} \sqrt{\Delta^{2}+\left(\alpha^{2}+\beta^{2}+2 \alpha \beta \sin 2 \theta\right) k^{2}}\right)}{k\left(\alpha e^{i \theta}+i \beta e^{-i \theta}\right)} .
\end{aligned}
$$

The GFs, $G(\vec{R}, 0, E)$ and $G(0, \vec{R}, E)$, can be obtained from Eq. (4.6), which becomes

$$
G_{\sigma_{1} \sigma_{2}}\left(\vec{r}_{1}, \vec{r}_{2}, E\right)=\frac{1}{A} \sum_{\vec{k} \nu} \frac{e^{i \vec{k} \cdot\left(\vec{r}_{1}-\vec{r}_{2}\right)} a_{\vec{k} \nu}^{\sigma_{1}} a_{\vec{k} \nu}^{\sigma_{2} *}}{E+i \eta-\varepsilon_{\vec{k} \nu}}
$$

$A$ being the area of the 2D space. From Eqs. (3.12) and (3.13) and the fact that changing $\vec{k}$ to $-\vec{k}$ is equivalent to changing the polar angle $\theta$ to $\theta+\pi$, one readily sees the following equalities:

$$
\varepsilon_{\nu}(\vec{k})=\varepsilon_{\nu}(-\vec{k}), a_{\vec{k} \nu}^{\uparrow}=-a_{-\vec{k} \nu}^{\uparrow} \text {, and } a_{\vec{k} \nu}^{\downarrow}=a_{-\vec{k} \nu}^{\downarrow}
$$

Using these results, it is easy to see that if we interchange $\vec{r}_{1}$ and $\vec{r}_{2}$ and change the dummy index $\vec{k}$ to $-\vec{k}$ in Eq. (3.14), the spin-diagonal part of the GF remains 
unchanged, while the off-diagonal part changes sign, viz.,

$$
\begin{aligned}
G_{\sigma \sigma}\left(\vec{r}_{1}, \vec{r}_{2}, E\right) & =G_{\sigma \sigma}\left(\vec{r}_{2}, \vec{r}_{1}, E\right), \\
\text { and } \quad G_{\sigma \sigma^{\prime}}\left(\vec{r}_{1}, \vec{r}_{2}, E\right) & =-G_{\sigma \sigma^{\prime}}\left(\vec{r}_{2}, \vec{r}_{1}, E\right) .
\end{aligned}
$$

Note that this is not a general result, but is valid only for specific spin texture of the wave function, and the conditions Eq. (3.15) must be satisfied under momentum inversion. Setting $\vec{r}_{2}=\vec{R}$ and $\vec{r}_{1}=0$ and substituting this equation into Eq. (3.7), we readily find our desired result for the GF coefficients, Eq. (3.11).

The expressions for the RKKY and the Dzyaloshinskii-Moriya interaction constants, Eq. (3.10), simplify to

$$
J=-\bar{\lambda} \int_{-\infty}^{E_{F}} \operatorname{Im}\left(g_{0}^{2}+g_{1}^{2}+g_{2}^{2}-g_{3}^{2}\right) d E,
$$

$\vec{D}=\left(D_{x}, D_{y}, 0\right)$ with

$$
\begin{aligned}
& D_{x}=-\bar{\lambda} \int_{-\infty}^{E_{F}} \operatorname{Re}\left(2 g_{0} g_{1}\right) d E, \\
& D_{y}=-\bar{\lambda} \int_{-\infty}^{E_{F}} \operatorname{Re}\left(2 g_{0} g_{2}\right) d E,
\end{aligned}
$$

and finally,

$$
\stackrel{\leftrightarrow}{\Gamma}=\left(\begin{array}{ccc}
\gamma_{x x} & \gamma_{x y} & 0 \\
\gamma_{y x} & \gamma_{y y} & 0 \\
0 & 0 & \gamma_{z z}
\end{array}\right)
$$


with

$$
\begin{aligned}
\gamma_{x x} & =\bar{\lambda} \int_{-\infty}^{E_{F}} \operatorname{Im}\left(2 g_{1}^{2}\right) d E \\
\gamma_{y y} & =\bar{\lambda} \int_{-\infty}^{E_{F}} \operatorname{Im}\left(2 g_{2}^{2}\right) d E \\
\gamma_{z z} & =-\bar{\lambda} \int_{-\infty}^{E_{F}} \operatorname{Im}\left(2 g_{3}^{2}\right) d E \\
\text { and } \gamma_{x y} & =\gamma_{y x}=\bar{\lambda} \int_{-\infty}^{E_{F}} \operatorname{Im}\left(2 g_{1} g_{2}\right) d E,
\end{aligned}
$$

where $\bar{\lambda} \equiv(2 \pi)^{-1} \lambda^{2} \hbar^{2}$. Note that the GF coefficients $g_{0}$ and $g_{i}$ are functions of $\vec{R}$ and $E$, being expansions of $G(\vec{R}, 0, E)$. In the next Sections, we use these expressions, Eqs. (3.17 - 3.20), to evaluate the magnetic interactions in the 2DEG described by the Hamiltonian Eq. (3.1).
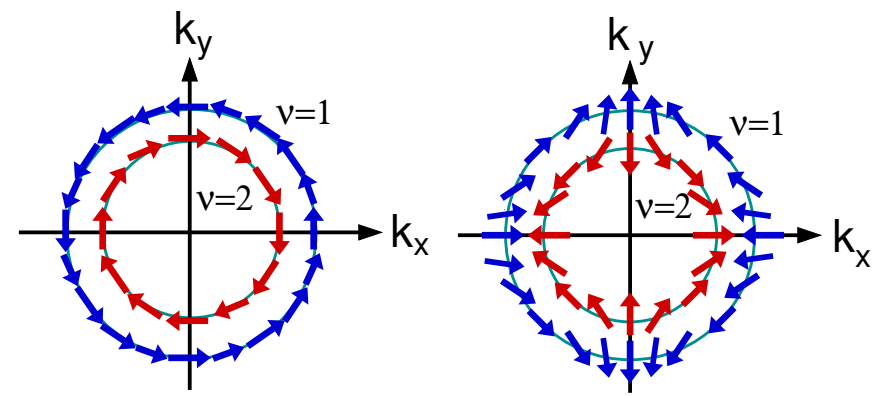

Figure 3.1: Spin eigenfunctions for the 2DEG with Rashba spin-orbit coupling. 


\subsection{DEG with Rashba spin-orbit coupling}

We consider first a 2DEG where only the Rashba interaction is present, with the remaining interactions (Zeeman and Dresselhaus) being zero $(\beta=\Delta=0)$. This is an important case found in many situations such as the semiconductor quantum wells[44] and oxide surfaces.[45] Diagonalizing the Hamiltonian Eq. (3.1) for this case

$$
H(\vec{k})=\left(\begin{array}{cc}
\frac{\hbar^{2} k^{2}}{2 m} & i \alpha k e^{-i \theta} \\
-i \alpha k e^{i \theta} & \frac{\hbar^{2} k^{2}}{2 m}
\end{array}\right),
$$

we have the eigenvalues and the eigenfunctions: $\varepsilon_{\vec{k} \nu}=\hbar^{2} k^{2} / 2 m \pm \alpha k, a_{\vec{k} \nu}^{\uparrow}=$ $\frac{i}{\sqrt{2}}(-1)^{\nu} e^{-i \theta}$, and $a_{\vec{k} \nu}^{\downarrow}=\frac{1}{\sqrt{2}}$ for the two bands $\nu=1$ and 2 , and $\theta=\tan ^{-1}\left(k_{y} / k_{x}\right)$, again, is the polar angle in the momentum space. From Eq. (4.6), the GF matrix elements $G_{\sigma \sigma^{\prime}}\left(\vec{r}_{1}, \vec{r}_{2}, E\right)$ are

$$
\begin{aligned}
& G_{\uparrow \uparrow}=G_{\downarrow \downarrow}=\frac{1}{2 A} \sum_{\vec{k}} e^{i \vec{k} \cdot\left(\vec{r}_{1}-\vec{r}_{2}\right)} \times\left(\frac{1}{\varepsilon_{+}}+\frac{1}{\varepsilon_{-}}\right), \\
& G_{\uparrow \downarrow}=\frac{i}{2 A} \sum_{\vec{k}} e^{i \vec{k} \cdot\left(\vec{r}_{1}-\vec{r}_{2}\right)} \times e^{-i \theta}\left(\frac{1}{\varepsilon_{+}}-\frac{1}{\varepsilon_{-}}\right), \\
& G_{\downarrow \uparrow}=-\frac{i}{2 A} \sum_{\vec{k}} e^{i \vec{k} \cdot\left(\vec{r}_{1}-\vec{r}_{2}\right)} \times e^{i \theta}\left(\frac{1}{\varepsilon_{+}}-\frac{1}{\varepsilon_{-}}\right),
\end{aligned}
$$

where

$$
\varepsilon_{ \pm}=E+i \mu-\left(\hbar^{2} k^{2} / 2 m \pm \alpha k\right) .
$$

The summations can be performed to yield the GF expansion coefficients $g_{i}$ by 
changing the summations into integrations and using the Jacobi-Anger expansion

$$
e^{i \vec{k} \cdot\left(\vec{r}_{1}-\vec{r}_{2}\right)}=J_{0}\left(k\left|\vec{r}_{1}-\vec{r}_{2}\right|\right)+2 \sum_{n=1}^{\infty} i^{n} J_{n}\left(k\left|\vec{r}_{1}-\vec{r}_{2}\right|\right) \cos (n \phi),
$$

where $J_{n}(x)$ is the $n$-th order Bessel function and $\phi$ is the angle between $\vec{r}_{1}-\vec{r}_{2}$ and $\vec{k}$. Since we have the rotational symmetry in the plane (the Rashba term can be written as $(\vec{k} \times \hat{z}) \cdot \vec{\sigma}$, so that only $\hat{z}$ is the only uniqe axis), we can choose $k_{x}$ along $\vec{r}_{1}-\vec{r}_{2}$ without any loss of generality. Thus $\phi=\theta$, and performing the angular integrations in Eq. (3.22) yields the GFs in terms of the Bessel functions, viz.,

$$
\begin{aligned}
& G_{\sigma \sigma}\left(\vec{r}_{1}, \vec{r}_{2}, E\right)=\frac{1}{4 \pi} \int_{0}^{\infty} k J_{0}\left(k\left|\vec{r}_{1}-\vec{r}_{2}\right|\right) \times\left(\frac{1}{\varepsilon_{+}}+\frac{1}{\varepsilon_{-}}\right), \\
& G_{\uparrow \downarrow}\left(\vec{r}_{1}, \vec{r}_{2}, E\right)=-\frac{1}{4 \pi} \int_{0}^{\infty} k J_{1}\left(k\left|\vec{r}_{1}-\vec{r}_{2}\right|\right) \times\left(\frac{1}{\varepsilon_{+}}-\frac{1}{\varepsilon_{-}}\right), \\
& G_{\downarrow \uparrow}\left(\vec{r}_{1}, \vec{r}_{2}, E\right)=-G_{\uparrow \downarrow}\left(\vec{r}_{1}, \vec{r}_{2}, E\right) .
\end{aligned}
$$

The diagonal elements are the same, while the off-diagonal elements differ by a sign, which leads to the form $G(\vec{R}, 0, E)=g_{0} \sigma_{0}+g_{2} \sigma_{2}, g_{1}$ and $g_{3}$ being both zero. One can similarly evaluate $G(0, \vec{R}, E)=g_{0} \sigma_{0}-g_{2} \sigma_{2}$, so that $G(\vec{R}, 0, E) \neq G(0, \vec{R}, E)$, a result consistent with the broken inversion symmetry. The momentum integrations 
in Eq. (3.25) after some algebra yield the coefficients:

$$
\begin{aligned}
g_{0}= & -\frac{i m}{4 \hbar^{2}}\left\{\frac{f(\alpha, E)+\alpha}{f(\alpha, E)} H_{0}^{(1)}\left[\frac{m}{\hbar^{2}}(f(\alpha, E)+\alpha) R\right]\right. \\
& \left.+\frac{f(\alpha, E)-\alpha}{f(\alpha, E)} H_{0}^{(1)}\left[\frac{m}{\hbar^{2}}(f(\alpha, E)-\alpha) R\right]\right\}, \\
g_{2}= & \frac{m}{4 \hbar^{2}}\left\{\frac{f(\alpha, E)+\alpha}{f(\alpha, E)} H_{1}^{(1)}\left[\frac{m}{\hbar^{2}}(f(\alpha, E)+\alpha) R\right]\right. \\
& \left.-\frac{f(\alpha, E)-\alpha}{f(\alpha, E)} H_{1}^{(1)}\left[\frac{m}{\hbar^{2}}(f(\alpha, E)-\alpha) R\right]\right\},
\end{aligned}
$$

where $f(\alpha, E)=\sqrt{\alpha^{2}+\frac{2 \hbar^{2}}{m} E}$, and $H_{n}^{(1)}(x)$ are the Hankel functions of the first kind and order $n$.

The magnetic interactions $J, \vec{D}$, and $\stackrel{\leftrightarrow}{\Gamma}$ are obtained from Eqs. $(3.17-3.19)$ and (3.26), and in light of the fact that only $g_{0}$ and $g_{2}$ are non-zero, this in turn makes only J, $D_{y}$, and $\gamma_{y y}$ non-zero, all other components being zero. These can be computed numerically in general, but for specific limits, approximate results may be obtained analytically, which we explore below.

It is straightforward to take the limit of the Rashba SOC going to zero $(\alpha=0)$ in Eq. (3.26) and obtain the well-known results for $J$ for the standard electron gas in 2D. [46]

It is instructive to show explicitly the rotational invariance of the interaction energy $E(\vec{R})$, i. e., if we rotate the position of the spin $\vec{R}$ as well as $\vec{S}_{1}, \vec{S}_{2}$ about the $\hat{z}$ axis, then the energy would not change, even though the individual interaction parameters might. This is true only if we have rotational symmetry in the plane, which is the case for the Rashba interaction.

To this end, we choose $\vec{R} \equiv \vec{r}_{2}-\vec{r}_{1}$ along the direction with polar angle $\gamma$ in the 
$k_{x}-k_{y}$ plane, and compute the coefficients $g_{i}$, which now depend on $\gamma$. The results, expressed in terms of the $\gamma$-independent quantities in Eq. (3.26), are: $g_{0}(\gamma)=g_{0}$, $g_{1}(\gamma)=-g_{2} \sin \gamma$, and $g_{2}(\gamma)=g_{2} \cos \gamma$. Putting these in Eqs. (3.17) - (3.20), we find the new interaction coefficients in terms of the old ones, viz., $J(\gamma)=J$, $D_{x}(\gamma)=-D_{y} \sin \gamma, D_{y}(\gamma)=D_{y} \cos \gamma, \gamma_{x x}(\gamma)=\gamma_{y y} \sin ^{2} \gamma, \gamma_{y y}(\gamma)=\gamma_{y y} \cos ^{2} \gamma$, $\gamma_{x y}(\gamma)=\gamma_{y x}(\gamma)=-\gamma_{y y} \sin \gamma \cos \gamma$, and rest of the coefficients are zero. After some matrix multiplications, one finds from Eq. (3.9) that the total energy $E(\vec{R})$ does not change under rotation, even though the individual interaction coefficients do change as just listed above.

\subsubsection{High Fermi-energy limit}

We consider the high Fermi energy $\left(E_{F} \gg m \alpha^{2} / \hbar^{2}\right)$ and long distance limit for $R$, so that the argument $x$ of the Hankel function in Eq. (3.26) is large, and the asymptotic expansion becomes

$$
H_{n}^{(1)}(x) \simeq 2^{1 / 2}(\pi x)^{-1 / 2} \exp [i(x-n \pi / 2-\pi / 4)] .
$$

This is valid for all energy except for $E \sim 0$, where the prefactor $x$ makes the limit $x H_{n}^{(1)}(x) \rightarrow x^{1 / 2}$, which therefore does not contribute much to the energy integrals in Eqs. (3.17 - 3.20). With this consideration, the large $x$ limit can be used in the entire range of energy $E$, and Eq. (3.26) yields in this limit the results

$$
\begin{aligned}
& g_{0} \simeq-i \bar{f} \times \exp \left[i\left(\frac{m}{\hbar^{2}} f(\alpha, E) R-\frac{\pi}{4}\right)\right] \cos \left(m \hbar^{-2} \alpha R\right), \\
& g_{2} \simeq \bar{f} \times \exp \left[i\left(\frac{m}{\hbar^{2}} f(\alpha, E) R-\frac{\pi}{4}\right)\right] \sin \left(m \hbar^{-2} \alpha R\right),
\end{aligned}
$$


where $\bar{f} \equiv m^{1 / 2}\left(2 \pi \hbar^{2} f(\alpha, E) R\right)^{-1 / 2}$, and we have assumed $f(\alpha, E) \gg \alpha$. Putting these expressions in Eqs. (3.17)- (3.20), we immediately find the long distance behavior

$$
\begin{aligned}
& J=-\frac{\lambda^{2} m}{8 \pi^{2} R^{2}} \cos \left(\frac{2 m \alpha}{\hbar^{2}} R\right) \sin \left(2 q_{F} R\right), \\
& \vec{D}=\hat{y} \frac{\lambda^{2} m}{8 \pi^{2} R^{2}} \sin \left(\frac{2 m \alpha}{\hbar^{2}} R\right) \sin \left(2 q_{F} R\right),
\end{aligned}
$$

and

$$
\gamma_{y y}=-\frac{\lambda^{2} m}{8 \pi^{2} R^{2}}\left(1-\cos \left(\frac{2 m \alpha}{\hbar^{2}} R\right)\right) \sin \left(2 q_{F} R\right)
$$

where

$$
q_{F}=\left(m^{2} \alpha^{2} / \hbar^{4}+2 m E_{F} / \hbar^{2}\right)^{1 / 2}
$$

This is consistent with the known results in the literature.[5]

There are several things to notice from these equations. First is that when $\alpha=0$, one recovers the limit for the standard 2DEG results[46] for J, and the vector and tensor interactions $\vec{D}$ and $\stackrel{\leftrightarrow}{\Gamma}$ become zero. Secondly, when one takes the small $\alpha$ limit, one gets the result that $J \propto \alpha^{0}, \vec{D} \propto \alpha$, and $\stackrel{\leftrightarrow}{\Gamma} \propto \alpha^{2}$, which indicates the appropriate power-law dependence on the strength of the $\operatorname{SOC} \alpha$. Finally, note that Eqs. (3.29)(3.31) show a beat pattern, which comes from the two different momentum scales in the problem, viz., the Fermi momentum for the two bands or their average $q_{F}$ and the difference $2 m \alpha / \hbar^{2}$, which appear in the equations.

To check the correctness of our approximations (3.27) and (3.28), in Fig. 3.2, we have plotted the magnitudes of the magnetic interactions computed with and without these approximations (in the later case Eqs. (3.26), and (3.17) - (3.20) were used). The similarity of these two curves confirms the approximations we have used 
in deriving the long-distance behavior Eqs. (3.29) - (3.31).

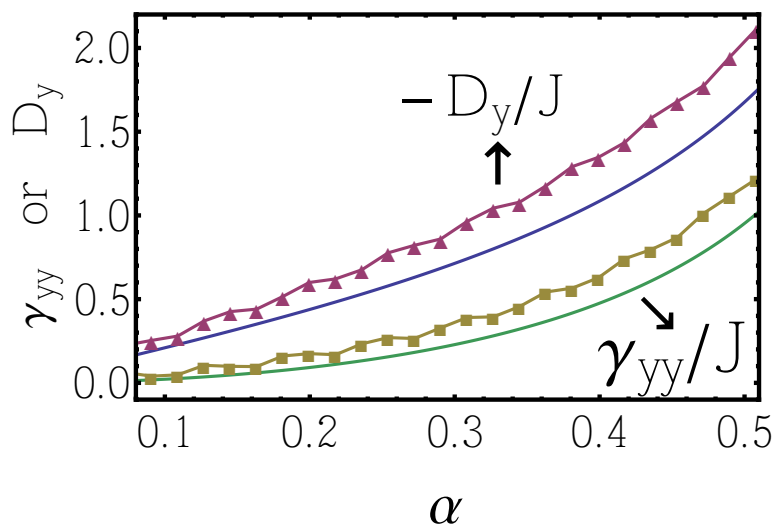

Figure 3.2: Numerical results for the ratio $D_{y} / J$ and $\gamma_{y y} / J$ as a function of $\alpha$ (in $\mathrm{eV} \cdot \AA)$ in the large $E_{F}$ and large $R$ limit. The points connected by lines indicate the numerical results, while the smooth lines are analytical results, Eqs. (3.29) - (3.31). Note from the same equations that the Fermi energy dependence drops out in the ratios, and here $R=7.5 \AA$ was used.

\subsubsection{Low Fermi-energy limit}

We consider now the limit of low Fermi energy $\left(\left|E_{F}-E_{\text {min }}\right| \ll\left|E_{\text {min }}\right|\right)$ and again the long-distance limit. In this case, the electrons occupy a small circular strip as indicated in Fig. (3.3), which resembles a one-dimensional system. Unsurprisingly, the system will show magnetic interactions characteristics of the 1DEG, as we shall see below. For this case, it is easy to see that

$$
f(\alpha, E) \ll \alpha .
$$

With this approximation, $\alpha$ can be neglected in the prefactors $f(\alpha, E) \pm \alpha$ appearing in the expressions for $g_{0}$ and $g_{2}$ in Eq. (3.26), but must be kept in the 


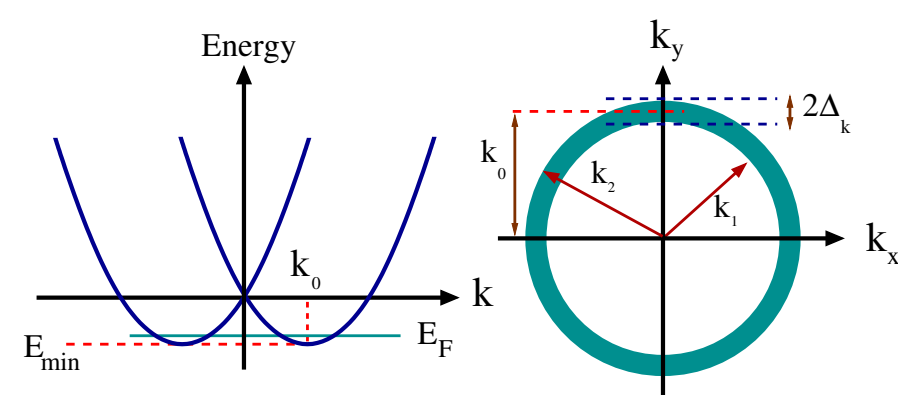

Figure 3.3: Occupied momentum states in the low- $E_{F}$ limit, valid for both cases of Rashba-only or Dresselhaus-only SOC. The occupied states form a thin circular strip, resembling a 1D electron gas. We have defined $\Delta_{k}=\left(k_{1}-k_{2}\right) / 2$ and $k_{0}=\left(k_{1}+k_{2}\right) / 2$, where $k_{0}=\alpha m / \hbar^{2}$ (Rashba) or $k_{0}=\beta m / \hbar^{2}$ (Dresselhaus).

arguments of the Hankel functions, which will produce the characteristic oscillations of the RKKY interactions as a function of $R$. Once again, for the long-distance limit, the asymptotic expansion (3.27) for the Hankel's functions can be used, leading to the result

$$
\begin{aligned}
& g_{0} \simeq-i \frac{m \alpha}{4 \hbar^{2}} \times \frac{1}{\sqrt{\pi \alpha R \varepsilon}} \times \\
& \left\{e^{i\left(\sqrt{\frac{2 m \varepsilon}{\hbar^{2}}} R+\frac{m \alpha}{\hbar^{2}} R-\pi / 4\right)}+i e^{i\left(\sqrt{\frac{2 m \varepsilon}{\hbar^{2}}} R-\frac{m \alpha}{\hbar^{2}} R-\pi / 4\right)}\right\},
\end{aligned}
$$

and

$$
\begin{aligned}
& g_{2} \simeq \frac{m \alpha}{4 \hbar^{2}} \times \frac{1}{\sqrt{\pi \alpha R \varepsilon}} \times \\
& \left\{e^{i\left(\sqrt{\frac{2 m \varepsilon}{\hbar^{2}}} R+\frac{m \alpha}{\hbar^{2}} R-3 \pi / 4\right)}-i e^{i\left(\sqrt{\frac{2 m \varepsilon}{\hbar^{2}}} R-\frac{m \alpha}{\hbar^{2}} R-3 \pi / 4\right)}\right\} .
\end{aligned}
$$

In the above equations, $\varepsilon \equiv E+\frac{m \alpha^{2}}{2 \hbar^{2}}$, which measures the energy with respect to the energy minimum in momentum space. The equations (3.17) - (3.20) then lead to the 
results in the low Fermi energy limit

$$
\begin{gathered}
J=\frac{\lambda^{2} m^{2} \alpha}{4 \pi^{2} \hbar^{2} R} \times \sin \left(2 k_{0} R\right) \operatorname{si}\left(2 \Delta_{k} R\right), \\
\vec{D}=-\hat{y} \frac{\lambda^{2} m^{2} \alpha}{4 \pi^{2} \hbar^{2} R} \times \cos \left(2 k_{0} R\right) \quad \operatorname{si}\left(2 \Delta_{k} R\right),
\end{gathered}
$$

and

$$
\gamma_{y y}=\frac{\lambda^{2} m^{2} \alpha}{4 \pi^{2} \hbar^{2} R} \times\left(\sin \left(2 k_{0} R\right)-1\right) \quad \operatorname{si}\left(2 \Delta_{k} R\right)
$$

where $\operatorname{si}(x)=S i(x)-\pi / 2, S i(x)$ being the well-known sine integral. Clearly, if $\alpha \rightarrow 0$, there are no electrons in the system, so that the magnetic interactions go to zero. Also, not surprisingly, the si $(x)$ function appears here, similar to the 1D case, since the Fermi surface forms a thin circular strip as seen from Fig. (3.3). Nevertheless, at large distances $\operatorname{si}(x) \propto 1 / x$, so that the magnetic interactions still fall off as $1 / R^{2}$, the well-know decay factor for RKKY interaction for the $2 \mathrm{D}$ case.[46]

\subsection{DEG with Dresselhaus spin-orbit coupling}

We consider the case when only the Dresselhaus term is present, so that the Hamiltonian is

$$
H(\vec{k})=\left(\begin{array}{cc}
\frac{\hbar^{2} k^{2}}{2 m} & \beta k e^{i \theta} \\
\beta k e^{-i \theta} & \frac{\hbar^{2} k^{2}}{2 m}
\end{array}\right),
$$

which leads to the eigenstates with energies $\varepsilon_{\vec{k} \nu}=\hbar^{2} k^{2} / 2 m+(-1)^{\nu} \beta k$ and the wave functions $|\vec{k} \nu\rangle=\left(a_{\vec{k} \nu}^{\uparrow}, a_{\vec{k} \nu}^{\downarrow}\right)=2^{-1 / 2}\left((-1)^{\nu} e^{i \theta}, 1\right)$ for $\nu=1$ and 2 , respectively. The GF coefficients for the Dresselhaus case can be expressed in terms of the same for Rashba 
case to yield

$$
g_{0}^{D}=g_{0}, g_{1}^{D}=-g_{2}, \text { and } g_{2}^{D}=g_{3}^{D}=0
$$

where in the expressions (3.26) for $g_{0}$ and $g_{2}, \alpha$ is to be replaced by $\beta$. Thus the algebra to get the magnetic interactions is the same as for the Rashba case, and one finds using Eqs. (3.17) - (3.20) that the magnetic interactions between the two cases are closely related.

Explicitly, one finds, in the high Fermi-energy, long-distance limit, the result

$$
\begin{aligned}
J & =-\frac{\lambda^{2} m}{8 \pi^{2} R^{2}} \cos \left(\frac{2 m \beta}{\hbar^{2}} R\right) \sin \left(2 q_{F} R\right), \\
\vec{D} & =-\hat{x} \frac{\lambda^{2} m}{8 \pi^{2} R^{2}} \sin \left(\frac{2 m \beta}{\hbar^{2}} R\right) \sin \left(2 q_{F} R\right),
\end{aligned}
$$

and

$$
\gamma_{x x}=-\frac{\lambda^{2} m}{8 \pi^{2} R^{2}}\left(1-\cos \left(\frac{2 m \beta}{\hbar^{2}} R\right)\right) \sin \left(2 q_{F} R\right)
$$

where $q_{F}=\left(m^{2} \beta^{2} / \hbar^{4}+2 m E_{F} / \hbar^{2}\right)^{1 / 2}$. Similarly, in the low Fermi-energy, longdistance limit, we get

$$
\begin{gathered}
J=\frac{\lambda^{2} m^{2} \beta}{4 \pi^{2} \hbar^{2} R} \times \sin \left(2 k_{0} R\right) \operatorname{si}\left(2 \Delta_{k} R\right), \\
\vec{D}=\hat{x} \frac{\lambda^{2} m^{2} \beta}{4 \pi^{2} \hbar^{2} R} \times \cos \left(2 k_{0} R\right) \operatorname{si}\left(2 \Delta_{k} R\right),
\end{gathered}
$$

and

$$
\gamma_{x x}=\frac{\lambda^{2} m^{2} \beta}{4 \pi^{2} \hbar^{2} R} \times\left(\sin \left(2 k_{0} R\right)-1\right) \operatorname{si}\left(2 \Delta_{k} R\right)
$$

Unlike the case of Rashba interaction in the last Section, here $\vec{D}$ is in the $\hat{x}$ direction, while only the $\gamma_{x x}$ termis non-zero in the tensor term $\stackrel{\leftrightarrow}{\Gamma}$. 
In writing the expression (3.40), we have, like in the case of Rashba, taken the distance vector $\vec{R}$ along the $k_{x}$ direction. If we take $\vec{R}$ along the polar angle $\gamma$, then the expressions for the GF coefficients become: $g_{0}^{D}(\gamma)=g_{0}^{D}, g_{1}^{D}(\gamma)=g_{1}^{D} \cos \gamma$, and $g_{2}^{D}(\gamma)=g_{1}^{D} \sin \gamma$, the rest being zero. However, interestingly, energy $E(\vec{R})$ here also does not depend on the direction of $\vec{R}$ in the plane.

\subsection{Spin-polarized 2DEG with no Rashba or Dres- selhaus term}

Another limit in which one can obtain the long-distance behavior for the magnetic interactions is the limit where there is spin polarization, but both the Rashba and the Dresselhaus SOC terms are absent, i. e., $\alpha=\beta=0$, but $\Delta \neq 0$. This case was treated in our earlier work, $[28,29]$ and we quote the results here for completeness.

In this case, the Green's function becomes spin-diagonal with the form

$$
G_{\sigma \sigma}\left(\vec{r}, \vec{r}^{\prime}, E\right)=-\frac{m}{\pi \hbar^{2}} K_{0}\left[-i \frac{\sqrt{2 m}}{\hbar}\left|\vec{r}-\vec{r}^{\prime}\right| \alpha(E \pm \Delta)\right]
$$

where $+(-)$ stands for $\sigma=\uparrow(\downarrow), K_{0}$ is the modified Bessel function of the second kind, and

$$
\alpha(x)= \begin{cases}\sqrt{x} & \text { if } x>0 \\ i \sqrt{|x|} & \text { if } x<0\end{cases}
$$


The only terms that survive are $J$ and $\gamma_{z z}$, and the expressions are

$$
\begin{gathered}
J=\frac{\lambda^{2} m^{2}}{8 \pi \hbar^{2}}\left\{-\frac{2}{\pi} \int_{-\Delta}^{\Delta} R e\left[K_{0}(\kappa R)\right] J_{0}\left(k_{+} R\right) d E\right. \\
\left.+\int_{\Delta}^{E_{F}}\left[J_{0}\left(k_{-} R\right) Y_{0}\left(k_{+} R\right)+Y_{0}\left(k_{-} R\right) J_{0}\left(k_{+} R\right)\right] d E\right\}, \\
\gamma_{z z}=\frac{\lambda^{2} m}{16 \pi R^{2}}\left[I^{\prime}\left(k_{F-} R\right)+I^{\prime}\left(k_{F+} R\right)\right]-J,
\end{gathered}
$$

where $I^{\prime}(x)=x^{2}\left[J_{0}(x) Y_{0}(x)+J_{1}(x) Y_{1}(x)\right]$. Even though the time reversal symmetry is broken, the inversion symmetry is still present, so that the DM interaction term $\vec{D}=0$. It can be easily shown that when the spin polarization $\Delta=0$, the only term that survives is $J$, and the expression becomes the same as the result for the standard 2DEG.[46]

\subsection{Magnetic interactions in the general case}

In the general case, when more than one of the three terms, $\alpha, \beta$, or $\Delta$, are non-zero, the Green's functions as well as the magnetic interactions must be calculated numerically using Eqs. (3.7), (3.13) -(3.14), and (3.17) - (3.20). We present below some numerical results. A case of particular interest is where $\alpha=\beta$ and $\Delta=0$, i.e., where the strengths of the Rashba and the Dresselhaus terms are the same. The system has $\mathrm{SU}(2)$ symmetry[47] and the Fermi surface as well as the spin eigenfunctions are shown in Fig. (3.4).

The expressions for the eigenstates are

$$
\varepsilon_{\vec{k} \nu}=\hbar^{2} k^{2} / 2 m+(-1)^{\nu} \sqrt{2} \alpha\left|k_{x}+k_{y}\right|
$$




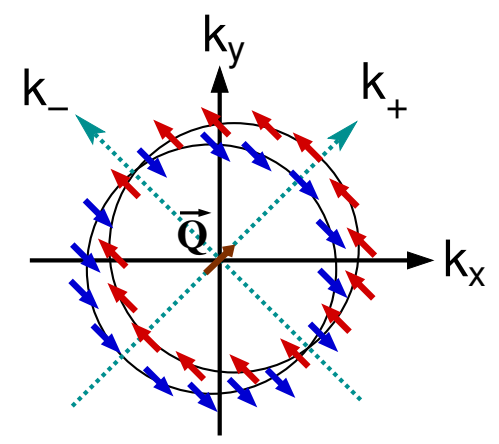

Figure 3.4: The spin eigenfunctions and the Fermi surface for the case $\alpha=\beta$ and $\Delta=0$, which consists of two circles with their centers shifted by the vector $\vec{Q}=$ $\left(Q_{x}, Q_{y}\right)=2 \sqrt{2} m \alpha(1,1)$. The two bands are indexed by $\nu=1$ for the outer shell and $\nu=2$ for the inner shell.
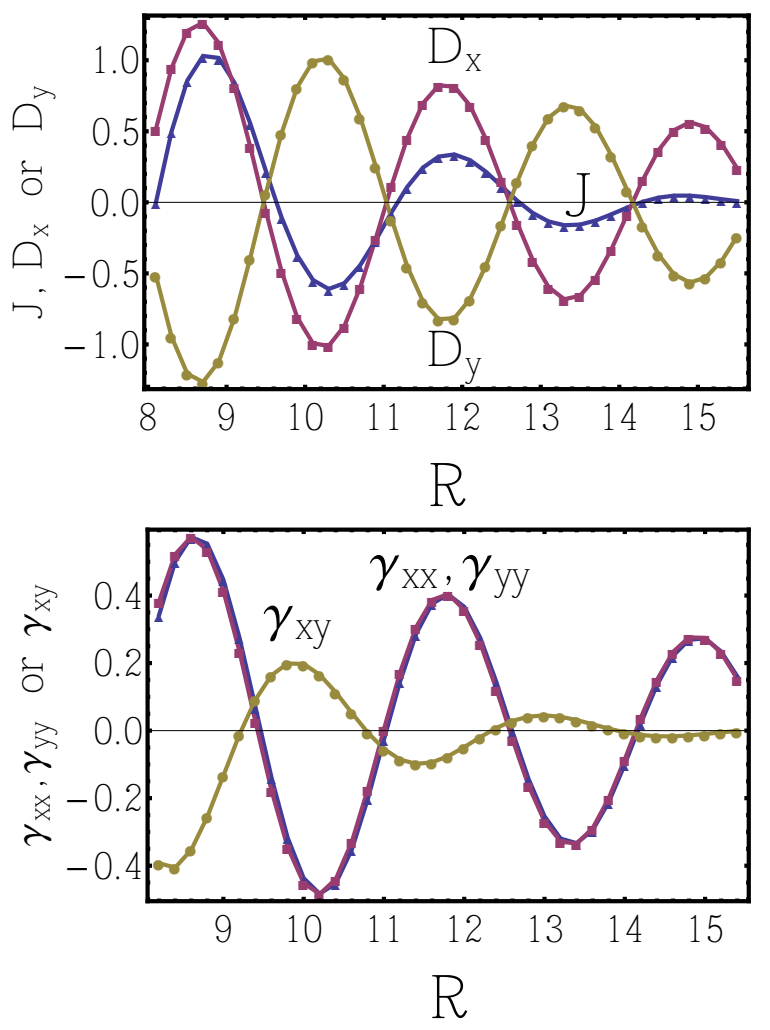

Figure 3.5: Computed magnetic interactions for equal Rashba and Dresselhaus SOC and $\Delta=0$. Here $E_{F}=3.4 \mathrm{eV}, \alpha=\beta=0.22 \mathrm{eV} \cdot \AA$, distance $R$ is in $\AA$, and the magnetic interactions are in units of $10^{-4} \times \lambda^{2} \mathrm{~m}$. 
and

$$
|\vec{k} \nu\rangle=\frac{1}{\sqrt{2}}\left((-1)^{\nu} \frac{1+i}{\sqrt{2}} \times \operatorname{sgn}\left(k_{x}+k_{y}\right)|\uparrow\rangle+|\downarrow\rangle\right),
$$

where sgn $(\mathrm{x})$ is the sign function. Putting these into Eq. (3.14) for the GF, one finds after straightforward calculations the following results for the GF coefficients:

$$
\begin{gathered}
g_{0}=\frac{1}{2 A} \sum_{\vec{k}} e^{i \vec{k} \cdot\left(\vec{r}_{1}-\vec{r}_{2}\right)}\left(\frac{1}{E+i \mu-\varepsilon_{+}^{\prime}}+\frac{1}{E+i \mu-\varepsilon_{-}^{\prime}}\right), \\
g_{2}=\frac{1}{2 \sqrt{2} A} \sum_{\vec{k}} e^{i \vec{k} \cdot\left(\vec{r}_{1}-\vec{r}_{2}\right)}\left(\frac{1}{E+i \mu-\varepsilon_{+}^{\prime}}-\frac{1}{E+i \mu-\varepsilon_{-}^{\prime}}\right), \\
g_{3}=0, \quad \text { and } g_{1}=-g_{2} .
\end{gathered}
$$

where $\varepsilon_{ \pm}^{\prime}=\hbar^{2} k^{2} / 2 m \pm \sqrt{2} \alpha\left(k_{x}+k_{y}\right)$. The general equations for the magnetic interactions Eq. (3.17) - (3.20) along with Eq. (3.53) lead to the results, viz., $D_{x}=$ $-D_{y}, \gamma_{x x}=\gamma_{y y}, \gamma_{z z}=0$ in this case, as may be seen from the computed interactions shown in Fig. (3.5).

\subsection{Domain Wall Energetics}

It is increasingly being realized that the DM interactions have an important effect in determining the domain wall structures in magnetic materials, particularly for ultrathin films, where surface and bulk anisotropy lead to the Rashba and Dresselhaus SOC, which in turn lead to the DM interactions. In an earlier work, Chen et al.[33] observed the formation of the Néel wall with a definite chirality in magnetized Ni/Co film, which was surprising since the Bloch wall usually has a lower energy. This was attributed to the existence of a strong DM interaction in the film. Furthermore, by 
interface engineering, they were able to adjust the DM interaction and stabilize either the left-handed or the right-handed Néel walls, or non-chiral Bloch walls. Indeed, in a more recent experiment, Di et al.[48] have directly measured a strong DM interaction in $\mathrm{Pt} / \mathrm{Co} / \mathrm{Ni}$ thin film structures using Brillouin spectroscopy. In addition, the strength of the DM interactions can be controlled by external means. For example, we have recently shown from ab initio calculations that the Rashba SOC can be tuned at the surface by an electric field.[45] Our work below does not refer to any specific material, but we make a few general observations on the domain wall energy based on our results in previous Sections.

We will consider the domain walls in ferromagnetic structures and the change in the wall energy due to the DM interactions. As first pointed out by Bloch, the domain wall thickness is determined by a competition between the exchange and the anisotropy energy. The wall structure, discussed in the seminal paper by Landau and Lifshitz, is determined by optimizing the total energy $E=\int_{-\infty}^{\infty}\left(A \dot{\theta}^{2}-B \cos ^{2} \theta\right) d x$, subject to the boundary condition, $\theta(-\infty)=0$, and $\theta(\infty)=\pi$, where $A$ is the exchange stiffness, $B$ is the anisotropy constant, $\theta(x)$ is the spin orientation across the domain wall, and $\dot{\theta} \equiv d \theta / d x$. The structure of the wall, obtained by solving the corresponding Euler-Lagrange equations, is given by

$$
\theta(x)=2 \tan ^{-1} \exp (2 x / L)
$$

where $L=2(A / B)^{1 / 2}$ is the width of the wall, and the result $\dot{\theta}(x)=\sqrt{B / A} \sin \theta(x)$ is used later in calculating the domain wall energy. We assume that the wall thickness does not change due to the DM interactions, which is reasonable because the DM interactions are considerably weaker as compared to the exchange and the anisotropy 
energies.

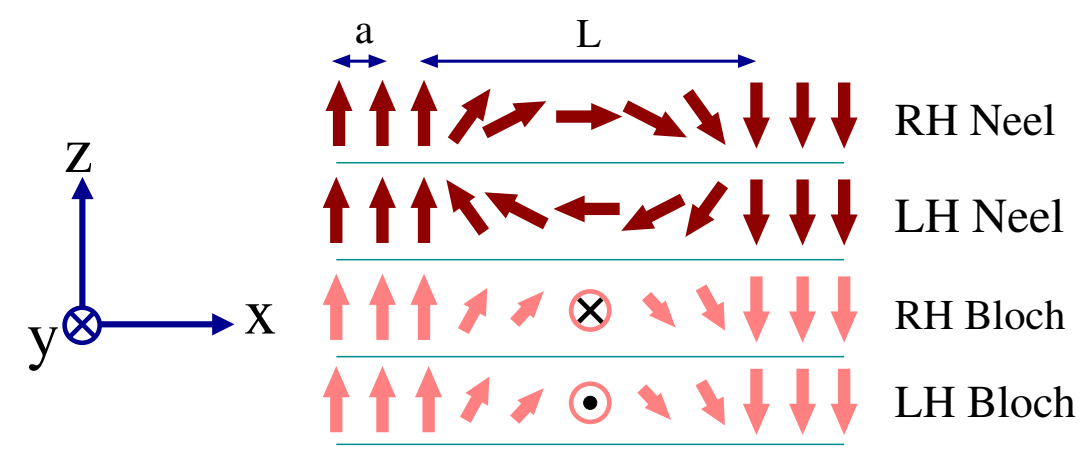

Figure 3.6: Right and left handed Néel and Bloch walls.

The spin orientations [see Fig. (3.6)] for the Néel and the Bloch walls, $\hat{S}_{N}^{R, L}(x)$ and $\hat{S}_{N}^{R, L}(x)$, where $R(L)$ refers to the right-handed (left-handed) wall are given by

$$
\begin{aligned}
& \hat{S}_{N}^{R, L}(x)=\cos \theta(x) \hat{z} \pm \sin \theta(x) \hat{x}, \\
& \hat{S}_{B}^{R, L}(x)=\cos \theta(x) \hat{z} \pm \sin \theta(x) \hat{y} .
\end{aligned}
$$

We compute the extra wall energy due to the DM terms for different walls from the total energy expression (3.9), the wall structure Eq. (3.54), and by keeping the dominant nearest-neighbor DM interactions for simplicity. To find the energy, we deal with different summations. For $J$ we need to compute

$$
\sum_{i} \cos (\theta(x+a)-\theta(x))=\sum_{i} \cos \left(a \frac{\theta(x)}{d x}\right) \simeq \sum_{i}\left(1-\frac{2 a}{L} \sin ^{2} \theta(x)\right)
$$

Here, we have used the facts that $\dot{\theta}^{2}=(B / A) \sin ^{2} \theta$ and $B / A=4 / L^{2}$. The first term in equation above leads to the $L / a=N_{w}$, and by using

$$
\sin ^{2} \theta=\frac{1-\cos (2 \theta)}{2}
$$


the second term leads to $-a / L=1 / N_{w}$. Here we have used the fact that $\sum_{i} \cos (2 \theta)=$ 0 . For the $\vec{S}_{1} \times \vec{S}_{2}$ term in the net interaction, we deal with $\sum_{i} \sin (\theta(x+a)-\theta(x))$ which is simply $\pi$. Next, we would like to calculate $\gamma_{x x}$ and $\gamma_{y y}$ terms. For both of these terms we have to evaluate

$$
\sum_{i} \sin \theta(x) \sin \theta(x+a)=\sum_{i} \sin \theta(x) \sin (a \dot{\theta}+\theta(x))
$$

Now one can use $\sin (\alpha+\beta)=\sin (\alpha) \cos (\beta)+\sin (\beta) \cos (\alpha)$ for the second term in equation above, the facts that $\sin (a \dot{\theta}) \simeq a \dot{\theta}$ and $\cos (a \dot{\theta}) \simeq 1-(a \dot{\theta})^{2} / 2$ to get the result of summation as

$$
\sum_{i} \sin \theta(x) \sin \theta(x+a)=\frac{N_{w}}{2}-\frac{3}{4 N_{w}} .
$$

To get this result, we have used the fact that $\sum_{i} \sin ^{2} \theta(x) \cos \theta(x)=0$. The last term in the interaction is related to $\gamma_{z z}$ term. Here we need to evaluate

$$
\sum_{i} \cos \theta(x) \cos \theta(x+a)=\sum_{i} \cos \theta(x) \cos (a \dot{\theta}+\theta(x))
$$

which can be easily found by using

$$
\cos (a \dot{\theta}+\theta(x))=\cos (a \dot{\theta}) \cos \theta(x)-\sin (a \dot{\theta}) \sin \theta(x)
$$

and the fact that $\sum_{i} \sin \theta(x) \cos ^{2} \theta(x)=0$. Here is the result

$$
\sum_{i} \cos \theta(x) \cos \theta(x+a)=\frac{N_{w}}{2}-\frac{1}{4 N_{w}} .
$$


Based on the fact that we have only one kind of wall in the system, $\gamma_{x y}$ and $\gamma_{y x}$ do not show up in the net energy. Finally, here is the result

$$
\begin{aligned}
& \frac{E_{N}^{R, L}}{S^{2}}=-\frac{J}{N_{w}} \pm \pi D_{y}+\frac{N_{w}}{2}\left(\gamma_{x x}-\gamma_{z z}\right)+\frac{3 \gamma_{x x}-\gamma_{z z}}{4 N_{w}} \\
& \frac{E_{B}^{R, L}}{S^{2}}=-\frac{J}{N_{w}} \mp \pi D_{x}+\frac{N_{w}}{2}\left(\gamma_{y y}-\gamma_{z z}\right)+\frac{3 \gamma_{y y}-\gamma_{z z}}{4 N_{w}}
\end{aligned}
$$

where $N_{w}=L / a$ is the size of the wall, and the upper/lower sign in \pm or $\mp$ refers to the right/left handed wall, and the interactions $J, D_{x}, D_{y}, \gamma_{i i}$ are between nearest neighbors. Comparing the relative energies of the two walls, we get

$$
\begin{aligned}
& \varepsilon_{N}^{R, L}= \pm \pi D_{y}+c \gamma_{x x}, \\
& \varepsilon_{B}^{R, L}=\mp \pi D_{x}+c \gamma_{y y},
\end{aligned}
$$

where $c=2^{-1} N_{w}+3\left(4 N_{w}\right)^{-1}$ is a constant. This result clearly shows that the helicity of the wall simply depends on the sign of the DM interaction $\vec{D}$, while the type of the wall (Bloch or Néel) depends on both $\vec{D}$ and $\stackrel{\leftrightarrow}{\Gamma}$. The energy expressions (3.64) suggest the tailoring of the domain wall structures by modifying the Rashba and Dresselhaus interactions

\subsection{Summary}

Here, we studied the indirect exchange interaction between two localized magnetic moments in the spin-polarized electron gas with both Rashba and Dresselhaus spinorbit coupling. Analytical expressions were found in certain limits. The magnitude 
of the vector and tensor DM interactions were estimated and compared to recent experiments on magnetic thin films. 


\section{Chapter 4}

\section{Impurity states in kagome lattice}

We study the electronic states introduced by impurities in the kagome lattice by applying the Green's function approach within a tight-binding model Hamiltonian. The impurities introduce localized states close to the Dirac point, in many ways similar to graphene, which will be discussed. In addition, we evaluate the interaction between two localized magnetic moments in the kagome lattice, study the effect of flat band in that interaction. The kagome lattice is a $2 \mathrm{D}$ lattice that has been of recent interest owing to its graphenelike band structure, the existence of flat band states and exotic quasiparticle excitations. Materials hosting the kagome lattice such as the rare-earth compounds and the herbertsmithites have been recently synthesized and efforts have been made to change the Fermi energy to the Dirac-like point by introducing impurities into this system.

Kagome lattice experimentally found in compounds is not pure, so studying effect

of an impurity in the kagome lattice is an interesting problem. Studying effect of an impurity in physical properties of a lattice automatically answers the questions on 
effect of a vacancy in the lattice structure, since a vacancy can be looked at a very strong impurity. A vacancy constitutes an important defect center, and for covalentlybonded crystals a strong effect on electronic and magnetic properties is expected. The electronic structure of a vacancy forms the basic foundation for understanding the behavior of more complex defects.

A number of theoretical studies of the electronic and magnetic properties of the vacancy in a honeycomb carbon lattice (graphene) have been reported in the past decade $[53,54,55,56,57,58,59,60,61,62,63,64,65,66]$. Based on the similarity of the lattice structure and the energy bands, we expect similar physics for the case of kagome lattice. What could be potentially interesting in this problem is effect of the flat band on both impurity states and wave functions, which, to the best of our knowledge, was never studied before. In the present work, we start from the fundamental equations in physics to find most general equations for the density of states and wave functions behaviors in the presence of an impurity. Then we use those formulas and some approximations to study this problem in a kagome lattice both computationally and analytically.

\subsection{Lattice structure}

Like the case of Graphene, kagome lattice has a triangular form, but in this case there are three atoms per unit cell. Using a nearest neighbor tight-binding model and the 
lattice structure shown in Fig. (4.1) one can find

$$
H(\vec{k})=\left(\begin{array}{ccc}
0 & 2 t \cos \left(\vec{k} \cdot \vec{\delta}_{1}\right) & 2 t \cos \left(\vec{k} \cdot \vec{\delta}_{2}\right) \\
2 t \cos \left(\vec{k} \cdot \vec{\delta}_{1}\right) & 0 & 2 t \cos \left(\vec{k} \cdot \vec{\delta}_{3}\right) \\
2 t \cos \left(\vec{k} \cdot \vec{\delta}_{2}\right) & 2 t \cos \left(\vec{k} \cdot \vec{\delta}_{3}\right) & 0
\end{array}\right)
$$

where $\vec{\delta}_{1}=\vec{t}_{1} / 2, \vec{\delta}_{2}=\vec{t}_{2} / 2$ and $\vec{\delta}_{3}=\vec{\delta}_{1}-\vec{\delta}_{2}$.

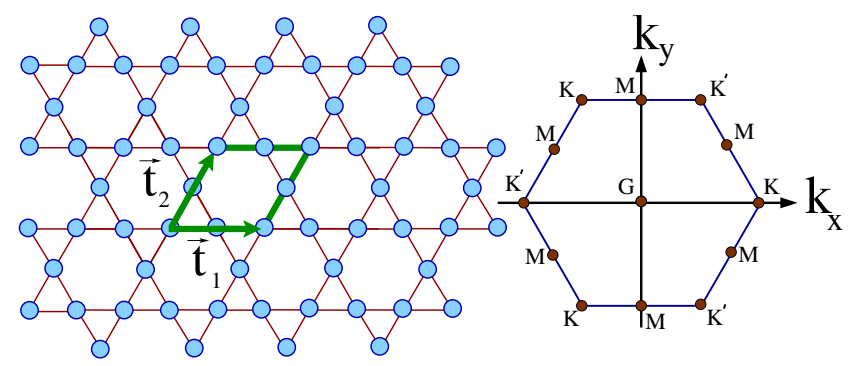

Figure 4.1: Kagome lattice and Brillouin zone. Here $\vec{t}_{1}=a(1,0)$ and $\vec{t}_{2}=a\left(\frac{1}{2}, \frac{\sqrt{2}}{3}\right)$. For future calculations, one can use $K=\frac{4 \pi}{3 a}(1,0), K^{\prime}=-K$ and $M=\frac{2 \pi}{\sqrt{3} a}(0,1)$. Based on the symmetry of the system, all of the atoms are equivalent.

The Hamiltonian defined in Eq. (4.1) leads to the eigenenergies

$$
\begin{aligned}
& \epsilon_{f}=-2 t \\
& \epsilon_{ \pm}=t \pm t \sqrt{4\left(\cos ^{2}\left(\vec{k} \cdot \vec{\delta}_{1}\right)+\cos ^{2}\left(\vec{k} \cdot \vec{\delta}_{2}\right)+\cos ^{2}\left(\vec{k} \cdot \vec{\delta}_{3}\right)\right)-3}
\end{aligned}
$$

As it can be seen, one of the bands doesn't have $k$-dependency and has been shown with $\epsilon_{f}$. This dispersionless flat band leads to very heavy and localized electrons. Using basic definition of electronic density of states (DOS), one can expect that the presence of the flat band in the electron band structure adds a delta-Dirac function to the DOS. We show this fact using both analytical and numerical approaches. On the other hand, two other bands will touch each other at the $K$ and $K^{\prime}$ points, as can 
be seen in Fig. (4.2). Expanding $\epsilon_{ \pm}$around the $K$ and $K^{\prime}$ points, it is convenient to find

$$
\epsilon_{ \pm}=t \pm t \frac{a \sqrt{3}}{2}|\vec{q}|
$$

where $|\vec{q}|$ is deviation from $K$ and $K^{\prime}$ points. Above equation which shows existence of Dirac cones, can be used when it comes to analytical calculations.

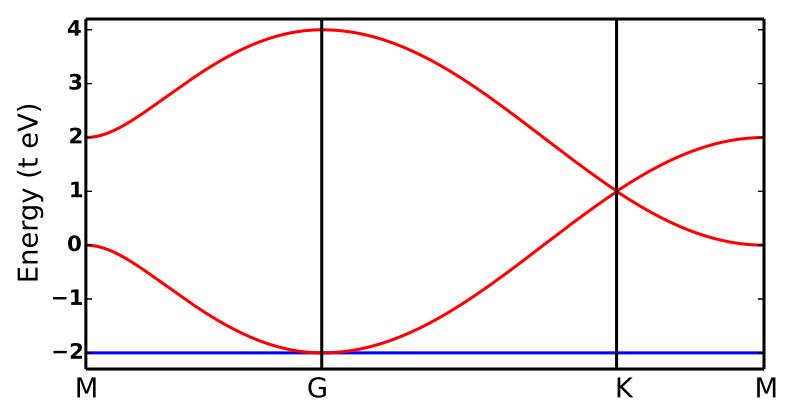

Figure 4.2: Tight binding band structure of the kagome lattice, with blue for the flat band and red for the dispersive bands.

Studying the electronic state introduced by a non-magnetic impurity and evaluating RKKY interaction in a system with both flat band and Dirac points are very interesting problems.

\subsection{Dyson's equation and impurity states}

To find the DOS as a function of energy, it is convenient to find the Green's function matrix in real space, using its operator $\hat{G}=(E+i \mu-\hat{H})^{-1}$, where $\hat{H}$ is our tightbinding Hamiltonian defined as

$$
\hat{H}=-t \sum c_{i \alpha}^{\dagger} c_{j \beta}+h . c .
$$


One needs to start from tight-binding model and our definition of Bloch function,

$$
|i \alpha\rangle=\frac{1}{\sqrt{N}} \sum_{i} e^{i \vec{k} \cdot \vec{r}_{i \alpha}}|i \alpha\rangle
$$

where $\alpha$ and $i$ in $\vec{r}_{i \alpha}$ denote the $\alpha$-th atom in the $i$-th unit cell and one can write it as $\vec{r}_{i \alpha}=\vec{R}_{i}+\vec{\tau}_{\alpha}$, with $\vec{R}_{i}$ and $\tau_{\alpha}$ as the position of $i$-th unit cell and $\alpha$-th atom. After some calculations, one can find the elements of the Green's function matrix in real space as

$$
\begin{aligned}
G_{i \alpha, j \beta}(E) & =\langle i \alpha|\hat{G}| j \beta\rangle \\
& =\frac{1}{N} \sum_{\vec{k}}^{\text {all }} e^{i \vec{k} \cdot\left(\vec{r}_{i \alpha}-\vec{r}_{j \beta}\right)} G_{\alpha \beta}(\vec{k}, E),
\end{aligned}
$$

where $G_{\alpha \beta}(\vec{k}, E)$ is the element of Green's function matrix in $\vec{k}$ space. This distance dependent form of the Green's function, Eq (4.6), can be use study impurity states and RKKY interaction.

The local DOS as a function of energy is simply defined as

$$
\operatorname{DOS}(E)=(-1 / \pi) \operatorname{Im}\left[\sum_{\alpha} G_{i \alpha, i \alpha}(E)\right]
$$

As we can see in Fig. (4.3), there is a delta-Dirac form for the DOS at flat band, $\epsilon=-2$. We provided analytical expressions for Green's function and local DOS for some two-dimesional lattices, including square, triangular, honeycomb and kagome lattices, in the Appendix. Our numerical calculations confirm reliability of formulas found analytically.

To study the effect of putting an impurity in the system, we locate an impurity 


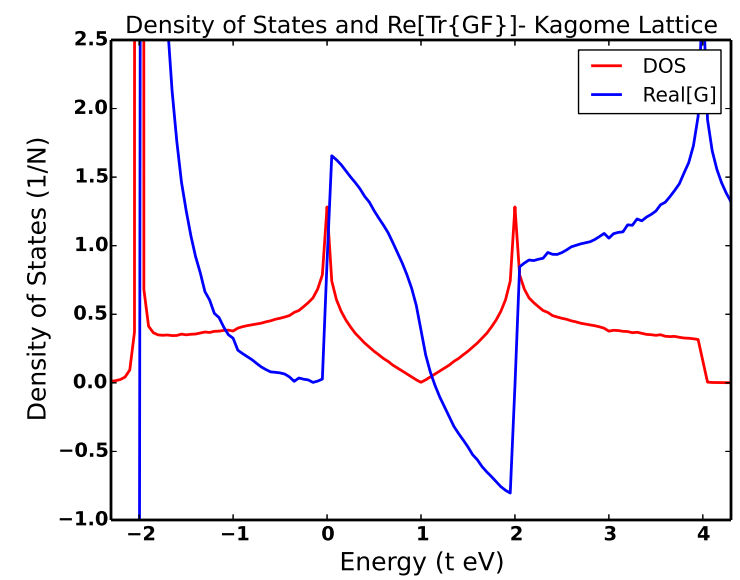

Figure 4.3: Numerical results for the real part of local trace of Green's function matrix and local DOS as a function of energy.

at the 0 -th unit cell at the position of $\gamma$-th atom, where $\gamma$ can be any of the three atoms in the unit cell. As we said, based on the symmetry of the system, all the atoms in the unit cell are equivalent, so it does not matter which atom we choose. The perturbing potential will have the form of $\hat{V}=U|0 \gamma\rangle\langle 0 \gamma|$, where $U$ is strength of our impurity. Note that if $U \rightarrow \infty$, it represents a vacancy. Showing the perturbed Green's function operator with $\hat{G}^{p}$ and using the Dyson's equation $\hat{G}^{p}=\hat{G}+\hat{G} \hat{V} \hat{G}^{p}$ we will get

$$
G_{i \alpha, i \alpha}^{p}(E)=G_{i \alpha, i \alpha}(E)+U G_{i \alpha, 0 \gamma}(E) G_{0 \gamma, i \alpha}^{p}(E)
$$

Now if we set left $i \alpha=0 \gamma$ in $\mathrm{Eq}(4.8)$, and then solve above equation for $G_{i \alpha, i \alpha}^{p}(E)$ we will get the elements of perturbed Green's function matrix in terms of unperturbed one, viz.,

$$
G_{i \alpha, i \alpha}^{p}(E)=G_{i \alpha, i \alpha}(E)+\frac{U G_{i \alpha, 0 \gamma}(E) G_{0 \gamma, i \alpha}(E)}{1-U G_{0 \gamma, 0 \gamma}(E)} .
$$

It is easy to see if $U \rightarrow 0$, then perturbed Green's function matrix goes to unperturbed one and so the local DOS. Eq (4.9) can be used to find local DOS at the position of 


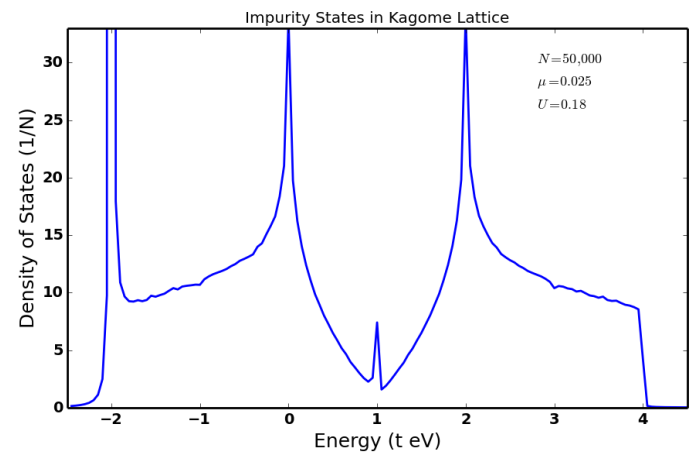

Figure 4.4: Numerical results for the impurity states. As it can be seen, presence of an impurity in the system changes the density around the $K$ and $K^{\prime}$ points.

any atom in the lattice when one puts an impurity at the 0-th unit cell at the position of $\gamma$-th atom. One just needs to use $\operatorname{DOS}(E)=(-1 / \pi) \operatorname{Im}\left[G_{i \alpha, i \alpha}^{p}(E)\right]$. Particularly, if one sets $i \alpha=0 \gamma$, we will find the local DOS at the position of impurity,

$$
\rho_{0 \gamma}^{p}(E)=\frac{-1}{\pi} \operatorname{Im}\left[G_{0 \gamma, 0 \gamma}^{p}(E)\right]=\frac{-1}{\pi} \operatorname{Im}\left[\frac{G_{0 \gamma, 0 \gamma}(E)}{1-U G_{0 \gamma, 0 \gamma}(E)}\right] .
$$

In the above equation if $U$ goes to infinity, then local DOS at the position of impurity will vanish, as we expect. Working with Eq. (4.10), one can write the perturbed density of state, $\rho_{0 \gamma}^{p}(E)$, at the position of perturbing impurity in terms of unperturbed DOS, $\rho_{0 \gamma}(E)$,

$$
\rho_{0 \gamma}^{p}(E)=\frac{\rho_{0 \gamma}(E)}{\left(1-U R e\left[G_{0 \gamma, 0 \gamma}(E)\right]\right)+U^{2} \pi^{2} \rho_{0 \gamma}^{2}(E)} .
$$

As we can see, resonance state happens when $\operatorname{Re}\left[G_{0 \gamma, 0 \gamma}(E)\right]=1 / U$. The total DOS at the 0-th unit cell, where we locate the impurity, can be found using $\rho_{\text {tot }}^{p}(E)=$ $\rho_{0 \gamma}^{p}(E)+\sum_{\alpha \neq \gamma} \rho_{0 \alpha}(E)$. Numerical results for DOS after putting an impurity in the system can be seen in Fig. (4.4). 
To find the analytical form of the Green's function matrix, we use the fact

$$
\begin{aligned}
G_{i \alpha, j \gamma}(E) & =\sum_{\vec{k} \nu}^{1 B Z} \frac{\langle i \alpha \mid \vec{k} \nu\rangle\langle\vec{k} \nu \mid j \gamma\rangle}{E+i \mu-\varepsilon_{\vec{k} \nu}} \\
& =\frac{1}{N} \sum_{\vec{k} \nu}^{1 B Z} \frac{e^{i \vec{k} \cdot\left(\vec{r}_{i}-\vec{r}_{j}\right)}\langle\vec{k} \alpha \mid \vec{k} \nu\rangle\langle\vec{k} \nu \mid \vec{k} \gamma\rangle}{E+i \mu-\varepsilon_{\vec{k} \nu}},
\end{aligned}
$$

where $|\vec{k} \nu\rangle$ are eigenstates of the original tight-binding Hamiltonian. We proceed the calculations by considering two approximations. First, we assume the system is doped so the Fermi energy is moved to $t$, and we assume the dispersive bands have linear form found in Eq. (4.3). In other words, we only consider the points around $K$ and $K^{\prime}$ points. Second, we assume the eigenstates at the $K$ and $K^{\prime}$ points are valid at entire first Brillouin zone.

\section{3 impurity state wave function}

To study the impurity state wave function we start from Lippmann-Schwinger equation,

$$
|\vec{k} \nu\rangle^{p}=|\vec{k} \nu\rangle+\hat{G} \hat{V}|\vec{k} \nu\rangle^{p}
$$

where $|\vec{k} \nu\rangle^{p}$ and $|\vec{k} \nu\rangle$ are perturbed and unperturbed states, respectively, and $\hat{V}$ and $\hat{G}$ are perturbing potential and unperturbed Green's function operators. What we want to do here is that we need to find impurity state wave function, defined as $\psi_{\vec{k} \nu}\left(\vec{r}_{i} \alpha\right)=\langle i \alpha \mid \vec{k} \nu\rangle^{p}$, in terms of unperturbed wave functions, $\varphi_{\vec{k} \nu}\left(\vec{r}_{i} \alpha\right)=\langle i \alpha \mid \vec{k} \nu\rangle$. Again, we put the impurity on the $\gamma$-th atom in the 0-th unit cell, so $\hat{V}=U|0 \gamma\rangle\langle 0 \gamma|$. 
Starting from Eq. (4.13) and multiplying both sides with $\langle i \alpha|$ we will get

$$
\psi_{\vec{k} \nu}\left(\vec{r}_{i} \alpha\right)=\varphi_{\vec{k} \nu}\left(\vec{r}_{i} \alpha\right)+U G_{i \alpha, 0 \gamma}(E) \psi_{\vec{k} \nu}(\overrightarrow{0} \gamma)
$$

If we set $i=0$ and $\alpha=\gamma$ in above equation and then solve it for $\psi_{\vec{k} \nu}(\overrightarrow{0} \gamma)$, we find

$$
\psi_{\vec{k} \nu}(\overrightarrow{0} \gamma)=\frac{\varphi_{\vec{k} \nu}(\overrightarrow{0} \gamma)}{1-U G_{0 \gamma, 0 \gamma}(E)}
$$

Inserting this result for $\psi_{\vec{k} \nu}(\overrightarrow{0} \gamma)$ in Eq. (4.14) leads to

$$
\psi_{\vec{k} \nu}\left(\vec{r}_{i} \alpha\right)=\varphi_{\vec{k} \nu}\left(\vec{r}_{i} \alpha\right)+\frac{U G_{i \alpha, 0 \gamma}(E)}{1-U G_{0 \gamma, 0 \gamma}(E)} \varphi_{\vec{k} \nu}(\overrightarrow{0} \gamma)
$$

One can use last result, found in Eq. (4.16), to study impurity state wave functions.

\subsection{Conclusion}

Here we studied impurity states for kagome lattice. The result we found here is very similar to the case of graphene as it was previously predicted. We found some numerical results for density of states in both presence and absence of an impurity. As an open problem, one can use some approximation and try to find analytical form for density of states and Green's function matrix. 


\section{Chapter 5}

\section{Summary}

In this dissertation, we focused on the interaction between two localized magnetic moments in a host solid. We have used Green's function methods and found the general form of interaction as

$$
E(\vec{R})=J \vec{S}_{1} \cdot \vec{S}_{2}+\vec{D} \cdot \vec{S}_{1} \times \vec{S}_{2}+\vec{S}_{1} \cdot \stackrel{\leftrightarrow}{\Gamma} \cdot \vec{S}_{2}
$$

Here $J, \vec{D}$ and $\stackrel{\leftrightarrow}{\Gamma}$ are functions of the distance between two localized magnetic moments $\vec{R}$, Fermi energy $E_{F}$ and strength of spin-orbit coupling factors. We found general formulas in terms of Green's function matrix elements which can be used to find $J, \vec{D}$ and $\gamma$, The Green's function matrix elements can be found using

$$
G_{\sigma_{1} \sigma_{2}}\left(\vec{r}_{1}, \vec{r}_{2}, E\right)=\sum_{\vec{k} \nu}^{\infty} \frac{\psi_{\vec{k} \nu}\left(\vec{r}_{1}, \sigma_{1}\right) \psi_{\vec{k} \nu}^{*}\left(\vec{r}_{2}, \sigma_{2}\right)}{E+i \eta-\varepsilon_{\vec{k} \nu}}
$$


Using this formalism, one can expand Green's function matrices in terms of Pauli matrices and perform the energy integration to find each term of the net interaction

$$
\begin{aligned}
& J=\frac{-\lambda^{2} \hbar^{2}}{2 \pi} \int_{-\infty}^{E_{F}} \operatorname{Im}\left(g_{0}^{\prime} g_{0}-\vec{g}^{\prime} \cdot \vec{g}\right) d E, \\
& \vec{D}=\frac{-\lambda^{2} \hbar^{2}}{2 \pi} \int_{-\infty}^{E_{F}} \operatorname{Re}\left(g_{0}^{\prime} \vec{g}-g_{0} \vec{g}^{\prime}\right) d E, \\
& \stackrel{\leftrightarrow}{\Gamma}=\frac{-\lambda^{2} \hbar^{2}}{2 \pi} \int_{-\infty}^{E_{F}} \operatorname{Im}\left(\vec{g} \vec{g}^{\prime}+\vec{g}^{\prime} \vec{g}\right) d E .
\end{aligned}
$$

The first term is known as RKKY and the last two terms are the vector and tensor Dzyaloshinskii-Moriya interactions. We have found analytical form for each term of the net interaction in the one-, two- and three-dimensional electron gas with broken time-reversal symmetries. Based on what we found, only broken inversion symmetry leads to vector Dzyaloshinskii-Moriya interaction, $\vec{D}$. In other words, to get the vector interaction, presence of spin-orbit coupling which leads to broken inversion symmetry is crucial. Broken time reversal symmetry, which is the case for spin-polarized electron gas, only leads to anisotropic form of RKKY interaction. In chapter 3, we brought Rashba and Dresselhaus spin-orbit coupling to the picture and applied our formalism to this case. We have found interesting analytical and numerical results for different limits.

The competition between RKKY and Dzyaloshinskii-Moriya terms lead to very interesting chiral magnetic structures, such as magnetic skyrmions, which are currently an area of considerable interest. The chiral structures originate from the Dzyaloshinskii-Moriya interactions caused by broken inversion symmetry and the presence of the spin-orbit interaction. As an application of our work, using those results, we found the energy of a Bloch and Neel domain wall. Using our model for 
energy of the wall, one can predict which one is preferred for given values of spin-orbit coupling.

In the last chapter, we have used Green's functions methods to find density of states introduced by an impurity in the kagome lattice. The kagome lattice has a flat band and also existence of Dirac-cone at the $K$-point makes it very interesting to study. Based on similarity of this problem and case of graphene, one can expect to see similar results for density of states. We found numerical results for change in the density of states and have studied it. To get analytical results, one needs to find analytical form of Green's function matrices and put them in expressions we found for density of states and impurity states. 


\section{Appendix A}

\section{General formula for RKKY and DM interactions using Lippmann-Schwinger equation}

Here, we derive a general expression for the interaction between two localized magnetic moments in a host material, known as RKKY and Dzyaloshinskii-Moriya interactions, using Lippmann-Schwinger equation[19]. Although the final result of this Appendix, Eq. (A.9), is known in the literature, working through this derivation is worthwhile. It can be useful for both beginners and experts in the field, and at the same time it provides some important information about the final result and its limitations.

First, we put the first localized magnetic moment $\vec{S}_{1}$ at $\vec{r}_{1}=\overrightarrow{0}$ and it perturbs the state of electrons, then second localized magnetic moment $\vec{S}_{2}$ will be located at $\vec{r}_{2}=\vec{R}$ and interacts with the perturbed electrons of the host.

Showing the perturbed and unperturbed states by $|\widetilde{k \nu}\rangle$ and $|k \nu\rangle$, respectively, 
one can use Lippmann-Schwinger equation to find

$$
|\widetilde{k \nu}\rangle \simeq|k \nu\rangle+\hat{G} \hat{V}_{1}|k \nu\rangle,
$$

where $\nu$ is the band index, $\hat{G}(E)=(E+i \mu-\hat{H})^{-1}$ with $\mu \rightarrow 0^{+}$, is the unperturbed retarded Green's function operator to be evaluated at $E=\varepsilon_{k \nu}$, and $\hat{V}_{i}$ indicates the perturbing potential operator applied by the $i$-th localized magnetic moment, and can be written as

$$
\hat{V}_{i}=-\lambda \sum_{\sigma \sigma^{\prime}}\left|\vec{r}_{i} \sigma\right\rangle\left\langle\vec{r}_{i} \sigma^{\prime}\right| \vec{S}_{i} \cdot \vec{s}_{\sigma \sigma^{\prime}}
$$

This perturbing potential has the contact interaction forms of $V_{1}(\vec{r})=-\lambda \delta(\vec{r}) \vec{S}_{1} \cdot \vec{s}$ and $V_{2}(\vec{r})=-\lambda \delta(\vec{r}-\vec{R}) \vec{S}_{2} \cdot \vec{s}$. Here, $\vec{s}$ is the spin of electrons and $\lambda$ is a phenomenological constant. In Eq. (A.2), $\vec{r}_{i}=\overrightarrow{0}$ and $\vec{R}$ for the first and second localized moments, respectively. Localized moment located at $\vec{R}$ interacts with the perturbed wavefunctions of the electrons, found in Eq. (A.1), so one can write the interaction total energy as $E=\sum_{\vec{k} \nu}^{o c c}\left\langle\widetilde{\vec{k} \nu}\left|\hat{V}_{2}\right| \widetilde{\vec{k}} \nu\right\rangle+\left\langle\vec{k} \nu\left|\hat{V}_{1}\right| \vec{k} \nu\right\rangle$, where occ stands for occupied and shows the fact that only occupied states will be taken into account. Writing this energy in the form of $E=E_{0}+E(\vec{R})$, we have

$$
\begin{aligned}
& E_{0}=\sum_{\vec{k} \nu}^{o c c}\left\langle\vec{k} \nu\left|\hat{V}_{1}+\hat{V}_{2}\right| \vec{k} \nu\right\rangle, \\
& E(\vec{R})=\sum_{\vec{k} \nu}^{o c c}\left\langle\vec{k} \nu\left|\hat{V}_{2} \hat{G} \hat{V}_{1}\right| \vec{k} \nu\right\rangle+\text { h.c. }=T+T^{*},
\end{aligned}
$$

where $E_{0}$ is a constant shift to the energy of the system $E(\vec{R})$ is the $\vec{R}$-dependent part of the total energy. For the systems without any broken symmetries or the cases 
that summation over all the spin of electrons is zero, in other words the system is not spin-polarized, $E_{0}$ will vanish. Based on this fact, we only focus on $E(\vec{R})$ which is the more interesting part of the total energy. Using the completeness relations: $\sum_{\sigma} \int d^{n} r=\sum_{\vec{r} \sigma}|\vec{r} \sigma\rangle\langle\vec{r} \sigma|=1$ and $\sum_{\vec{k} \sigma}|\vec{k} \sigma\rangle\left\langle\vec{k} \sigma\left|=\sum_{\vec{k} \nu}\right| \vec{k} \nu\right\rangle\langle\vec{k} \nu|=1$, and defining $\psi_{\vec{k} \nu}(\vec{r}, \sigma)=\langle\vec{r} \sigma \mid \vec{k} \nu\rangle$ we find

$$
T=\lambda^{2} \sum_{\vec{k} \nu}^{o c c} \sum_{\sigma_{1} \sigma_{2}} \psi_{\vec{k} \nu}^{*}\left(\vec{R}, \sigma_{1}\right)\left\langle\sigma_{1}\left|\vec{S}_{2} \cdot \vec{s} G\left(\vec{R}, 0, \varepsilon_{\vec{k} \nu}\right) \vec{S}_{1} \cdot \vec{s}\right| \sigma_{2}\right\rangle \psi_{\vec{k} \nu}\left(0, \sigma_{2}\right) .
$$

In the above equation, $G\left(\vec{R}, 0, \varepsilon_{\vec{k} \nu}\right)$ is the retarded Green's function matrix evaluated at $E=\varepsilon_{\vec{k}, \nu}$ which its elements can be found using

$$
G_{\sigma_{1} \sigma_{2}}\left(\vec{r}_{1}, \vec{r}_{2}, E\right)=\sum_{\vec{k} \nu}^{\infty} \frac{\psi_{\vec{k} \nu}\left(\vec{r}_{1}, \sigma_{1}\right) \psi_{\vec{k} \nu}^{*}\left(\vec{r}_{2}, \sigma_{2}\right)}{E+i \mu-\varepsilon_{\vec{k} \nu}}
$$

with $\mu \rightarrow 0^{+}$. We will now use the facts $G\left(\vec{R}, 0, \varepsilon_{\vec{k} \nu}\right)=\int_{-\infty}^{\infty} G(\vec{R}, 0, E) \delta\left(E-\varepsilon_{\vec{k} \nu}\right)$ and $\int_{-\infty}^{\infty} d E \times \sum_{\vec{k} \nu} \rightarrow \int_{-\infty}^{E_{F}} d E \times \sum_{\vec{k} \nu}^{o c c}$ to write $T$ in the form of

$T=\lambda^{2} \int_{-\infty}^{E_{F}} d E \sum_{\sigma_{1} \sigma_{2}}\left\langle\sigma_{1}\left|\vec{S}_{2} \cdot \vec{s} G(\vec{R}, 0, E) \vec{S}_{1} \cdot \vec{s}\right| \sigma_{2}\right\rangle \sum_{\vec{k} \nu}^{\infty} \psi_{\vec{k} \nu}^{*}\left(\vec{R}, \sigma_{1}\right) \psi_{\vec{k} \nu}\left(0, \sigma_{2}\right) \delta\left(E-\varepsilon_{\vec{k} \nu}\right)$.

Here, there is an important point: to get the final result we have changed the order of integration over $\vec{k}$ and $E$ in Eq. (A.6). As it has been said in the paper, if Fubini's condition $[20,21]$ is not satisfied, changing the order of integration in a double-integral can change the result of integration. It is important to emphasize that when one uses the last result of this Appendix, Eq. (A.9), to find the magnetic interaction, it should be carefully evaluated, and in the case that the result does not make physical sense, 
the method of integration over $\vec{k}$, which is using $E(\vec{R})=2 R e[T]$ with $T$ defined in the Eq. (A.4), should be used.

The $\delta$-function in Eq. (A.6) can be expressed in a form that it is useful to make Green's function. The equality $\lim _{\mu \rightarrow 0}(x \pm i \mu)^{-1}=P\left(x^{-1}\right) \mp i \pi \delta(x)$ leads to the fact $\delta(x)=(i / 2 \pi) \lim _{\mu \rightarrow 0}\left((x+i \mu)^{-1}-(x-i \mu)^{-1}\right)$. Expanding $\delta\left(E-\varepsilon_{\vec{k} \nu}\right)$ in Eq. (A.6) in that form, and using $\sum_{\sigma}|\sigma\rangle\langle\sigma|=1$ and $E(\vec{R})=2 R e[T]$ with $T$ defined in the Eq. (A.4) result the $\vec{R}$-dependent part of the energy as

$$
\begin{aligned}
E(\vec{R})= & \frac{-\lambda^{2}}{\pi} \operatorname{Im} \int_{-\infty}^{E_{F}} \operatorname{Tr}\left[G(0, \vec{R}, E) \vec{S}_{2} \cdot \vec{s} G(\vec{R}, 0, E) \vec{S}_{1} \cdot \vec{s}\right] d E \\
& +\frac{\lambda^{2}}{\pi} \operatorname{Im} \int_{-\infty}^{E_{F}} \operatorname{Tr}\left[G^{A}(0, \vec{R}, E) \vec{S}_{2} \cdot \vec{s} G(\vec{R}, 0, E) \vec{S}_{1} \cdot \vec{s}\right] d E
\end{aligned}
$$

where $G^{A}(0, \vec{R}, E)$ is Advanced Green's function matrix which its elements can be found using

$$
G_{\sigma_{1} \sigma_{2}}^{A}\left(\vec{r}_{1}, \vec{r}_{2}, E\right)=\sum_{\vec{k} \nu}^{\infty} \frac{\psi_{\vec{k} \nu}\left(\vec{r}_{1}, \sigma_{1}\right) \psi_{\vec{k} \nu}^{*}\left(\vec{r}_{2}, \sigma_{2}\right)}{E-i \mu-\varepsilon_{\vec{k} \nu}}
$$

with $\mu \rightarrow 0^{+}$. It is convenient to show that second term in Eq. (A.7) turns out to be zero. To show this, one can easily expand the Green's function matrices in terms of Pauli matrices, then use the fact that $G^{A}(0, \vec{R}, E)=G^{\dagger}(\vec{R}, 0, E)$. The last fact leads to the desired result of this Appendix,

$$
E(\vec{R})=\frac{-\lambda^{2}}{\pi} \operatorname{Im} \int_{-\infty}^{E_{F}} \operatorname{Tr}\left[G(0, \vec{R}, E) \vec{S}_{2} \cdot \vec{s} G(\vec{R}, 0, E) \vec{S}_{1} \cdot \vec{s}\right] d E
$$




\section{Appendix B}

\section{RKKY Interaction for the Spin Polarized Electron Gas Using the Method of Summation Over Momentum}

The Ruderman-Kittel-Kasuya-Yosida (RKKY) interaction between two magnetic moments embedded in a solid was originally derived for the free electron gas, $[1,2,3]$ and it has been extensively studied since then. It has the Heisenberg form $J \vec{S}_{1} \cdot \vec{S}_{2}$ and shows an oscillatory behavior as a function of the distance $R$ between the two magnetic moments; for example, $J(R) \propto(x \cos x-\sin x) / x^{4}$ for the electron gas in three dimensions (3D), where $x=2 k_{F} R$ with $k_{F}$ being the Fermi momentum. Using lattice models, Dzyaloshinskii and Moriya (DM) showed that $[16,17]$ in situations with broken symmetry (inversion or time reversal), there appears a vector or a tensor interaction term, $\vec{D}$ and $\stackrel{\leftrightarrow}{\Gamma}$, respectively, in addition to the scalar, RKKY interaction $J$, so that the net interaction energy is given by the Hamiltonian 


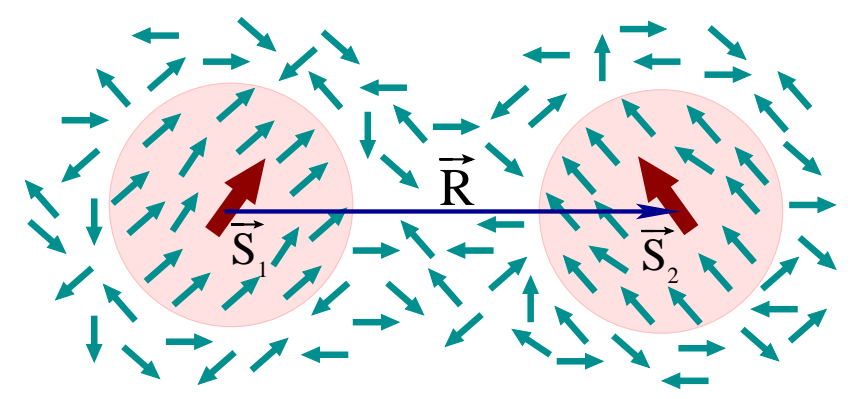

Figure B.1: (Color online) The interaction between two magnetic moments mediated by the host electrons in the crystal, which leads to the well known RKKY interaction of the type $J \vec{S}_{1} \cdot \vec{S}_{2}$. However, if the host crystal has broken symmetry, then additional interaction terms appear, known as the Dzyaloshinskii-Moriya interaction.

$\mathcal{H}=J \vec{S}_{1} \cdot \vec{S}_{2}+\vec{D} \cdot \vec{S}_{1} \times \vec{S}_{2}+\vec{S}_{1} \cdot \stackrel{\leftrightarrow}{\Gamma} \cdot \vec{S}_{2}$. There is currently a considerable interest on this less-studied DM interactions, because of the recent observation[12, 13, 14] of skyrmions, a novel vortex-like spin structure[11], that forms due to the competition between the RKKY and the DM terms.

While the RKKY interaction is quite simply illustrated by taking the example of the free electron gas, this is not the case for the DM interactions. The original work of DM involved lattice models, while in many subsequent works, complicated interactions such as non-magnetic spin-flip centers[49] or relativistic scattering potentials[50] were used to obtain the DM interactions. In this paper, we point out that the simple generalization of the electron gas to include spin polarization does indeed produce the DM interaction, albeit just the tensor part as the vector part $\vec{D}=0$ because the inversion symmetry is still present.

Before we discuss the case of the spin-polarized electron gas, we note that the coupling between the spin and orbital degrees of freedom is essential to the DM interactions, as without it electron energies are spin degenerate, $\varepsilon(k \uparrow)=\varepsilon(k \downarrow)$, and it turns out that, if this is true, then there would only be the RKKY interaction. 
This is the case for the standard, non-spin-polarized electron gas. If there is sufficient symmetry, even in the presence of spin-orbit coupling, the electron states may still be spin degenerate, so that the presence of the spin-orbit coupling alone is not enough to produce the DM interaction.

In general, the presence of the inversion symmetry in a solid leads to the condition

$$
\varepsilon_{\vec{k} \uparrow}=\varepsilon_{-\vec{k} \uparrow}
$$

while the time reversal symmetry implies that

$$
\varepsilon_{\vec{k} \uparrow}=\varepsilon_{-\vec{k} \downarrow}
$$

so that if both symmetries are present, one obtains the spin-degenerate energies, viz., $\varepsilon_{\vec{k} \uparrow}=\varepsilon_{\vec{k} \downarrow}$. This equality is violated if there is at least one broken symmetry. The simplest way to include such a broken symmetry is to consider spin polarized bands in a 3D electron gas, which has broken time reversal symmetry. This may for example be produced by a magnetic field, either externally applied or produced by the constituent atoms in the solid. We now consider the interaction between magnetic moments placed in the spin-polarized electron gas.

In this Appendix, we extend the original work of Ruderman, Kittel, Kasuya, and Yosida (RKKY) on the interaction between two magnetic moments embedded in an electron gas to the case where the electron gas is spin polarized. The broken symmetry of a host material introduces the Dzyaloshinskii-Moriya (DM) vector and tensor interaction terms, in addition to the standard RKKY term, so that the net interaction energy has the form: $\mathcal{H}=J \vec{S}_{1} \cdot \vec{S}_{2}+\vec{D} \cdot \vec{S}_{1} \times \vec{S}_{2}+\vec{S}_{1} \cdot \stackrel{\leftrightarrow}{\Gamma} \cdot \vec{S}_{2}$. We find 
that for the spin-polarized electron gas, a non-zero tensor interaction $\stackrel{\leftrightarrow}{\Gamma}$ is present in

addition to the scalar RKKY interaction $J$, while $\vec{D}$ is zero due to the presence of inversion symmetry. Explicit expressions for these are derived for the electron gas both in 2D and 3D and we show that the net magnetic interaction can be expressed as a sum of Heisenberg and Ising like terms. The RKKY interaction exhibits a beating pattern, caused by the presence of the two Fermi momenta $k_{F \uparrow}$ and $k_{F \downarrow}$, while the $R^{-3}$ distance dependence of the original RKKY result for the 3D electron gas is retained. This model serves as a simple example of the magnetic interaction in systems with broken symmetry, which goes beyond the RKKY interaction.

\section{B.1 Spin-polarized electron gas and magnetic in- teraction}

The magnetic interaction energy between the two localized moments is obtained by using the quantum mechanical perturbation theory. The first magnetic moment perturbs the electron gas, which is then felt by the second moment, thereby coupling the two moments. The unperturbed eigenstates of the electron gas are plane wave states

$$
\begin{aligned}
& |\vec{k} \sigma\rangle=\frac{1}{\sqrt{\Omega}} e^{i \vec{k} \cdot \vec{r}}|\sigma\rangle, \\
& \varepsilon_{\vec{k} \sigma}=\frac{\hbar^{2} k^{2}}{2 m} \mp \Delta,
\end{aligned}
$$

where we have taken the energies to be spin dependent with the $\mp$ sign corresponding to the energy of the spin $\uparrow$ and $\downarrow$ states, respectively, and the box normalization has been used with $\Omega$ being the volume of the box. We now introduce two localized spins 
$\vec{S}_{1}$ and $\vec{S}_{2}$, located at the origin and at the position $\vec{R}$, respectively, which interact with the electron gas via the contact interaction

$$
\begin{aligned}
& V_{1}(\vec{r})=-\lambda \delta(\vec{r}) \vec{S}_{1} \cdot \vec{s} \\
& V_{2}(\vec{r})=-\lambda \delta(\vec{r}-\vec{R}) \vec{S}_{2} \cdot \vec{s}
\end{aligned}
$$

where $\vec{s}$ is the spin of the electrons, and, in operator form, the perturbing potentials may be written as $\hat{V}_{1}=-\lambda|\vec{r}\rangle\langle\vec{r}| \vec{S}_{1} \cdot \vec{s}$ and similarly for $\hat{V}_{2}$.

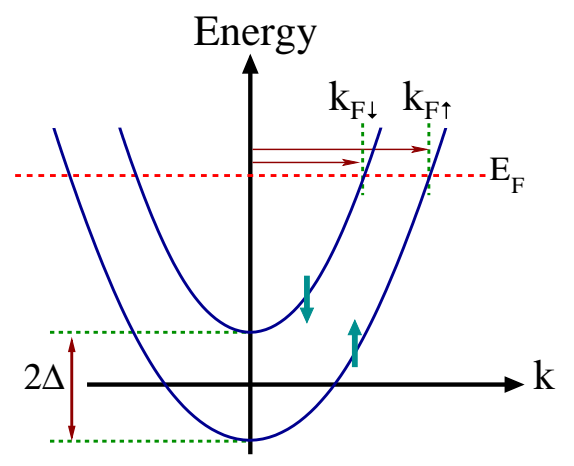

Figure B.2: (Color online) Electron gas with spin dependent energy. $E_{F}$ denotes the Fermi energy and the Fermi momenta in the two spin channels are indicated by $k_{F \uparrow}$ and $k_{F \downarrow}$.

The perturbed electron states $|\vec{k} \sigma\rangle\rangle$ due to the first moment may be expressed in terms of the Lippmann-Schwinger equation[19], correct to the first order in perturbation theory

$$
|\vec{k} \sigma\rangle\rangle=|\vec{k} \sigma\rangle+\hat{G} \hat{V}_{1}|\vec{k} \sigma\rangle
$$

where $\hat{G}(E)=(E+i \mu-\hat{H})^{-1}$ with $\mu \rightarrow 0^{+}$is the unperturbed retarded Green's function to be evaluated at the energy $E=\varepsilon_{\vec{k} \sigma}$, and $\hat{H}$ is the unperturbed Hamiltonian that produces the eigenstates in Eq. (B.3). Eq. (B.5) may be written in terms of the 
matrix elements of the Green's function, which reads

$$
|\vec{k} \sigma\rangle\rangle=|\vec{k} \sigma\rangle-\frac{\lambda}{\sqrt{\Omega}} \int d^{3} r \sum_{\sigma^{\prime}}\left|\vec{r} \sigma^{\prime}\right\rangle G_{\sigma \sigma^{\prime}}\left(\vec{r}, 0, \varepsilon_{\vec{k} \sigma}\right) \vec{S}_{1} \cdot \vec{s}_{\sigma \sigma^{\prime}}
$$

The Green's function in the present case is diagonal in the spin indices

$$
\begin{aligned}
& G_{\sigma \sigma^{\prime}}\left(\vec{r}, \vec{r}^{\prime}, E\right) \equiv\left\langle\vec{r} \sigma|\hat{G}(E)| \vec{r}^{\prime} \sigma^{\prime}\right\rangle \\
& =\frac{1}{\Omega} \sum_{\vec{k}} \frac{e^{i \vec{k} \cdot\left(\vec{r}-\vec{r}^{\prime}\right)}}{E+i \mu-\varepsilon_{\vec{k}, \sigma}} \delta_{\sigma \sigma^{\prime}} .
\end{aligned}
$$

The spin $\vec{S}_{2}$ at $\vec{R}$ feels the perturbation via the contact interaction $V_{2}(r)$, leading to the interaction energy

$$
E=\sum_{\vec{k} \sigma}^{o c c}\left\langle\left\langle\vec{k} \sigma\left|\hat{V}_{2}\right| \vec{k} \sigma\right\rangle\right\rangle=E_{0}+E(\vec{R})+O\left(\lambda^{3}\right) .
$$

Note that in general the distance dependent term $E(\vec{R})$ depends on the vector distance $\vec{R}$ separating the two magnetic moments; however, the isotropic nature of the electron gas considered here makes it depend only on the magnitude $R$, so that from now on we write it as $E(R)$. Equation (B.8) is evaluated by substituting Eq. (B.6) for the perturbed states $|\vec{k} \sigma\rangle\rangle$ and performing the summation over the occupied states. The $E_{0}$ term, which is $O(\lambda)$, introduces a constant shift in energy; In fact there is an additional $O(\lambda)$ term, viz., $\left\langle\vec{k} \sigma\left|\hat{V}_{1}\right| \vec{k} \sigma\right\rangle$, originating from the perturbation of the first spin to the electron gas. Adding this to Eq. (B.8) to redefine $E_{0}$, and performing a straightforward momentum summation, it is easy to show that, for the $3 \mathrm{D}$ electron 
gas,

$$
E_{0}=\frac{-\hbar \lambda}{12 \pi^{2}}\left(S_{1 z}+S_{2 z}\right)\left(k_{F \uparrow}^{3}-k_{F \downarrow}^{3}\right)
$$

A similar expression for the $2 \mathrm{D}$ electron gas is given later.

This result is easily interpreted, viz., that $E_{0}$ is the product of the energy gained per spin-up electron $\varepsilon_{0}$ times the excess number $N$ of spin up electrons over spin down electrons, since the energy gain of a spin-up electron is exactly negative of that of a spin-down electron for each of the terms in Eq. (B.4). The energy gain for a spin-up electron is simply: $\varepsilon_{0}=\left\langle k \uparrow\left|V_{1}+V_{2}\right| k \uparrow\right\rangle=-(\lambda \hbar / 2 \Omega) \times\left(S_{1 z}+S_{2 z}\right)$, as computed for a single plane-wave electron, Eq. (B.3), using the first-order perturbation theory with the perturbation Eq. (B.4). The excess number of spin up electrons is $N=$ $(4 \pi / 3)\left(k_{F \uparrow}^{3}-k_{F \downarrow}^{3}\right) /\left(8 \pi^{3} / \Omega\right)$, which is the difference of the Fermi surface volumes for the two spins divided by the amount of momentum space $8 \pi^{3} / \Omega$ per distinct electron state.[51] Putting these two together, we immediately arrive at the expression Eq. (B.9) using $E_{0}=n \epsilon_{0}$. Note that we have used the standard Pauli matrices for the itinerant electron spins, e.g., $s_{z}=(\hbar / 2)\left(\begin{array}{cc}1 & 0 \\ 0 & -1\end{array}\right)$, etc., so that effectively we have chosen $\hat{z}$ as the spin quantization axis and this is why the $z$ components $S_{1 z}$ and $S_{2 z}$ appear in Eq. (B.9).

The next term in Eq. (B.8), which is $O\left(\lambda^{2}\right)$, produces the magnetic interaction and may be written as

$$
E(R)=\frac{\lambda^{2}}{\Omega} \sum_{\vec{k} \sigma}^{o c c}\left\langle\sigma\left|\vec{S}_{2} \cdot \vec{s} G\left(\vec{R}, 0, \varepsilon_{\vec{k} \sigma}\right) \vec{S}_{1} \cdot \vec{s}\right| \sigma\right\rangle e^{-i \vec{k} \cdot \vec{R}}+c . c .
$$

Now, as mentioned already, $G_{\sigma^{\prime} \sigma^{\prime \prime}}\left(\vec{R}, 0, \varepsilon_{\vec{k} \sigma}\right)$ is diagonal in the spin space, though it 
can be different for $\sigma=\uparrow$ or $\downarrow$ for a given $\vec{k}$. It is convenient to write them in terms of the Pauli matrices:

$$
\begin{aligned}
& G\left(\vec{R}, 0, \varepsilon_{\vec{k} \uparrow}\right)=g_{0} \sigma_{0}+\frac{1}{2} u\left(\sigma_{0}-\sigma_{z}\right), \\
& G\left(\vec{R}, 0, \varepsilon_{\vec{k} \downarrow}\right)=g_{0} \sigma_{0}+\frac{1}{2} d\left(\sigma_{0}+\sigma_{z}\right),
\end{aligned}
$$

where $g_{0}, u$, and $d$ are c-numbers and functions of $\vec{k}$ and $\vec{R}$. Putting this into the energy expression Eq. (B.10), we now get

$$
\begin{aligned}
& E(R)=\frac{\lambda^{2}}{\Omega}\left(\sum_{\vec{k} \sigma}^{o c c}\left\langle\sigma\left|\vec{S}_{2} \cdot \vec{s} g_{0} \sigma_{0} \vec{S}_{1} \cdot \vec{s}\right| \sigma\right\rangle e^{-i \vec{k} \cdot \vec{R}}\right. \\
& +\sum_{k=0}^{k_{F \uparrow}}\left\langle\uparrow\left|\vec{S}_{2} \cdot \vec{s}\left(g_{0} \sigma_{0}+\frac{u}{2}\left(\sigma_{0}-\sigma_{z}\right)\right) \vec{S}_{1} \cdot \vec{s}\right| \uparrow\right\rangle e^{-i \vec{k} \cdot \vec{R}} \\
& \left.+\sum_{k=0}^{k_{F \downarrow}}\left\langle\downarrow\left|\vec{S}_{2} \cdot \vec{s}\left(g_{0} \sigma_{0}+\frac{d}{2}\left(\sigma_{0}+\sigma_{z}\right)\right) \vec{S}_{1} \cdot \vec{s}\right| \downarrow\right\rangle e^{-i \vec{k} \cdot \vec{R}}\right)+ \text { c.c. }
\end{aligned}
$$

This can be evaluated using the spin identities

$$
\begin{aligned}
& \operatorname{Tr}\left[\vec{S}_{1} \cdot \vec{\sigma} \vec{S}_{2} \cdot \vec{\sigma}\right]=2 \vec{S}_{1} \cdot \vec{S}_{2}, \\
& \left\langle\uparrow\left|\vec{S}_{1} \cdot \vec{\sigma} \vec{S}_{2} \cdot \vec{\sigma}\right| \uparrow\right\rangle=\vec{S}_{1} \cdot \vec{S}_{2}+i\left(\vec{S}_{1} \times \vec{S}_{2}\right)_{z}, \\
& \left\langle\downarrow\left|\vec{S}_{1} \cdot \vec{\sigma} \vec{S}_{2} \cdot \vec{\sigma}\right| \downarrow\right\rangle=\vec{S}_{1} \cdot \vec{S}_{2}-i\left(\vec{S}_{1} \times \vec{S}_{2}\right)_{z}, \\
& \left\langle\uparrow\left|\vec{S}_{1} \cdot \vec{\sigma} \sigma_{z} \vec{S}_{2} \cdot \vec{\sigma}\right| \uparrow\right\rangle=-\vec{S}_{1} \cdot \vec{S}_{2}-i\left(\vec{S}_{1} \times \vec{S}_{2}\right)_{z}+2 S_{1 z} S_{2 z}, \\
& \left\langle\downarrow\left|\vec{S}_{1} \cdot \vec{\sigma} \sigma_{z} \vec{S}_{2} \cdot \vec{\sigma}\right| \downarrow\right\rangle=\vec{S}_{1} \cdot \vec{S}_{2}-i\left(\vec{S}_{1} \times \vec{S}_{2}\right)_{z}-2 S_{1 z} S_{2 z},
\end{aligned}
$$


and the energy expression Eq. (B.12) can be cast into the desired form

$$
E(R)=J \vec{S}_{1} \cdot \vec{S}_{2}+\vec{D} \cdot\left(\vec{S}_{1} \times \vec{S}_{2}\right)+\vec{S}_{1} \cdot \stackrel{\leftrightarrow}{\Gamma} \cdot \vec{S}_{2}
$$

where the various coefficients may be obtained by converting the summations into integrations in Eq. (B.12).

Note from the expressions above, Eqs. (B.12 - B.14), that the strengths of the RKKY and the DM interaction terms $(J, \vec{D}$, and $\stackrel{\leftrightarrow}{\Gamma})$ are comparable, all being proportional to the square of the size of the interaction $\lambda$ between the itinerant and the localized spins. This is different from the original DM interaction,[17] where two atoms in a solid interact with each other via an exchange interaction path that involves the spin-orbit coupled excited states on the two atoms. In this case, $J \sim \xi^{0}, \vec{D} \sim \xi$, and $\stackrel{\leftrightarrow}{\Gamma} \sim \xi^{2}$, where $\xi$ is the spin-orbit coupling strength, so that $|\stackrel{\leftrightarrow}{\Gamma}| \ll|\vec{D}| \ll|J|, \xi$ being a small parameter, and it is then customary to ignore the tensor DM interaction $\stackrel{\leftrightarrow}{\Gamma}$. In the spin-polarized electron gas case, treated here, the interaction between the two localized spins is mediated via the perturbation of the electron gas and no spinorbit split states are involved in the exchange path; so that it is entirely a different mechanism. This means that if a spin-polarized electron gas is present in addition to atoms with spin-orbit coupling, both effects must be considered separately and the dominant effect for the DM vector and tensor interactions might as well come from the perturbation of the electron gas, of the type studied in this paper. 


\section{B.2 Results}

\section{B.2.1 Electron gas in 3D}

We now evaluate the interaction energy coefficients in Eq. (B.14) for the spin polarized electron gas in 3D by evaluating the Green's function and performing the momentum integrations. A straightforward contour integration[52] of the expression (B.7) yields the Green's function

$$
G(\vec{r}, 0, E)=\frac{-2 m}{4 \pi r \hbar^{2}}\left(\begin{array}{cc}
e^{i \alpha(E+\Delta) r} & 0 \\
0 & e^{i \alpha(E-\Delta) r}
\end{array}\right)
$$

where

$$
\alpha(x)=\left\{\begin{array}{l}
\left(2 m \hbar^{-2} x\right)^{1 / 2} \text { if } x>0, \\
i\left(2 m \hbar^{-2}|x|\right)^{1 / 2} \text { if } x<0 .
\end{array}\right.
$$

Comparing Eq. (B.15) with Eq. (B.11), we have: $g_{0}=\beta e^{i k R}, u=\beta e^{-\sqrt{2 \delta^{2}-k^{2}} R}-g_{0}$, if $-\Delta<\varepsilon_{\vec{k} \uparrow}<\Delta$, or $\beta e^{i \sqrt{k^{2}-2 \delta^{2}} R}-g_{0}$, if $\varepsilon_{\vec{k} \uparrow}>\Delta$, and $d=\beta e^{i \sqrt{k^{2}+2 \delta^{2}} R}-g_{0}$, if $\varepsilon_{\vec{k} \downarrow}>\Delta$. Here $\beta=-m\left(2 \pi \hbar^{2} r\right)^{-1}$. Plugging these values into Eq. (B.12), converting the momentum summations into integrations in the same equation, and performing the integrations, we finally get, using the identities Eq. (B.13), the result for the RKKY interaction

$$
J=-\frac{\lambda^{2} m}{(4 \pi)^{3} R^{4}} \times\left(I\left(x_{\uparrow}\right)+I\left(x_{\downarrow}\right)\right),
$$

where $x_{\uparrow}=k_{F \uparrow} R, x_{\downarrow}=k_{F \downarrow} R$, and $I(x)=\sin (2 x)-2 x \cos (2 x)$. As anticipated, the vector DM interaction $\vec{D}=0$, because of the presence of inversion symmetry, while 
the tensor interaction survives:

$$
\stackrel{\leftrightarrow}{\Gamma}=\left(\begin{array}{lll}
P & 0 & 0 \\
0 & P & 0 \\
0 & 0 & 0
\end{array}\right),
$$

where $P=g\left(x_{\downarrow}, x_{\uparrow}\right)-J\left(x_{\downarrow}, x_{\uparrow}\right)$ and defining $\eta=\left(x_{\uparrow}^{2}-x_{\downarrow}^{2}\right)^{1 / 2}=\sqrt{2} \delta R$, the expression for $g$ is

$$
\begin{aligned}
g\left(x_{\downarrow}, x_{\uparrow}\right) & =-\frac{\lambda^{2} m}{8 \pi^{3} R^{4}}\left(\int_{0}^{\eta} e^{-\sqrt{\eta^{2}-x^{2}}}+\int_{\eta}^{x_{\uparrow}} \cos \sqrt{x^{2}-\eta^{2}}\right. \\
& \left.+\int_{0}^{x_{\downarrow}} \cos \sqrt{x^{2}+\eta^{2}}\right) \times x \sin x d x .
\end{aligned}
$$

We note that for the unpolarized electron gas, putting $k_{F \uparrow}=k_{F \downarrow}=k_{F}$, the above expressions yield $P=0$, so that we recover the standard RKKY result: $E(R)=$ $J \vec{S}_{1} \cdot \vec{S}_{2}$, where $J=-\lambda^{2} m /\left(32 \pi^{3} R^{4}\right) I\left(k_{F} R\right)$. We illustrate the oscillatory behavior of $J$ and $P$ in Fig. (B.3) for typical parameters. The beating pattern seen in the figure comes from the interference of the two Fermi momenta for the two spins. For example, it is easy to see from Eq. (B.17) that for large distances $J \sim R^{-3} \cos \left(k_{F \uparrow} R+\right.$ $\left.k_{F \downarrow} R\right) \times \cos \left(k_{F \uparrow} R-k_{F \downarrow} R\right)$, which leads to the beating pattern as indicated in Fig. (B.3).

To estimate the size of the oscillations in Fig. B.3, we consider a real material iron. Even though iron is hardly a model for the ideal spin-polarized electron gas, it provides us with a simple system to estimate the order of magnitude of the oscillation periods. Taking the Fermi energy and the spin splittings from band calculations for iron,[31] $E_{F} \approx 8 \mathrm{eV}$ and $2 \Delta \approx 2 \mathrm{eV}$, we find the oscillation periods of the beating 

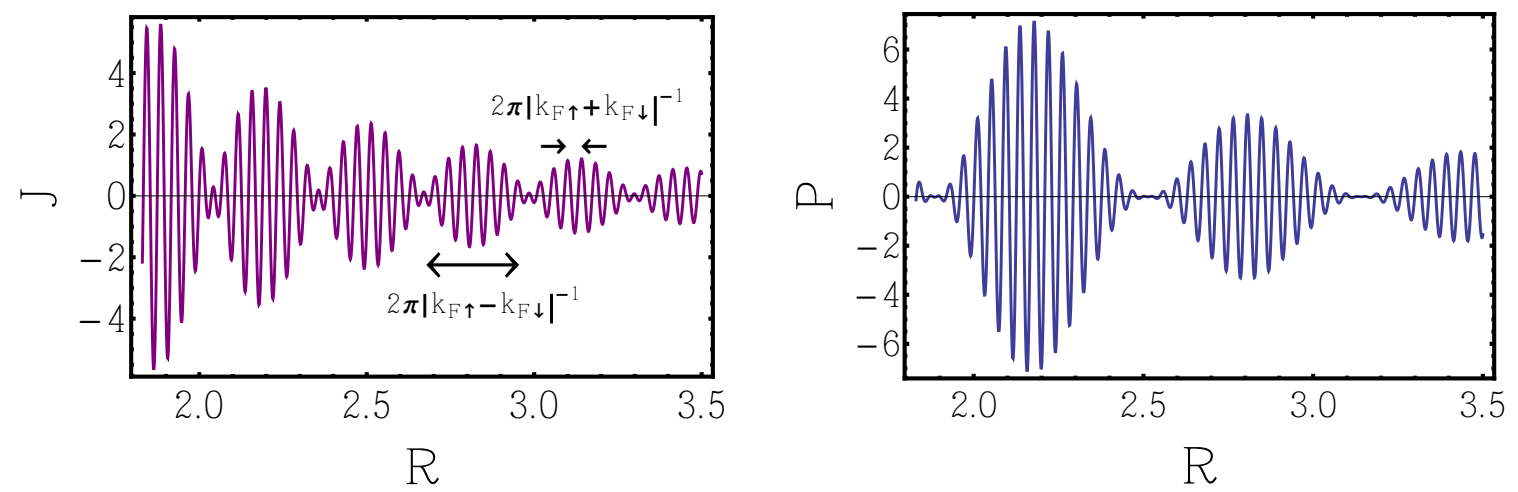

Figure B.3: (Color online) The RKKY interaction $J$ (top) and the tensor Dzyaloshinskii-Moriya interaction $P$ (bottom), in units of $m \pi^{-3} \lambda^{2}$, for the 3D electron gas as a function of distance $R$, obtained from Eqs. (B.17 - B.19).

patterns to be: $2 \pi\left|k_{F \uparrow}+k_{F \downarrow}\right|^{-1} \approx 1.5 \AA$ and $2 \pi\left|k_{F \uparrow}-k_{F \downarrow}\right|^{-1} \approx 50 \AA$.

Note that the constant energy shift $E_{0}$, Eq. (B.9), is proportional to $\lambda \delta k_{F}$, where $\delta k_{F}=k_{F \uparrow}-k_{F \downarrow}$ is the spin polarization at the Fermi surface, while the magnetic interactions, Eqs. (B.17 - B.19), are proportional to the second order $\lambda^{2}$. Thus, the magnetic interaction terms dominate over the constant energy shift for the weakly spin-polarized case, viz., $\delta k_{F}<<\left(m k_{F}^{2}\right) \lambda$, where $k_{F}=\left(k_{F \uparrow}+k_{F \downarrow}\right) / 2$ is the spinaveraged Fermi momentum and the typical scenario, $\delta k_{F}<<k_{F}$, has been assumed. Finally, note from the form of the tensor interaction Eq. (B.18) that the net interaction Eq. (B.14) can be written as a sum of the Heisenberg and Ising interactions,

$$
E(R)=J^{\prime} \vec{S}_{1} \cdot \vec{S}_{2}+J^{\prime \prime} S_{1 z} S_{2 z}
$$

where $J^{\prime}=J+P$ and $J^{\prime \prime}=-P$. This is true both for the $3 \mathrm{D}$ electron gas as well as for the 2D gas described below. 


\section{B.2.2 Electron Gas in 2D}

In 2D, the Green's function Eq. (B.7) becomes

$$
G_{\sigma \sigma^{\prime}}\left(\vec{r}, \vec{r}^{\prime}, E\right)=\frac{\delta_{\sigma \sigma^{\prime}}}{(2 \pi)^{2}} \int \frac{e^{i \vec{k} \cdot\left(\vec{r}-\vec{r}^{\prime}\right)}}{E+i \mu-\varepsilon_{\vec{k}, \sigma}} d^{2} k .
$$

Using the Jacobi-Anger expansion[25] in terms of the Bessel's functions, viz., $e^{i \vec{k} \cdot \vec{r}}=$ $J_{0}(k r)+2 \sum_{n=1}^{\infty} i^{n} J_{n}(k r) \cos (n \theta)$, where $\theta$ is measured with respect to $\hat{r}$, the angular integration produces just one non-zero term involving $J_{0}(k r)$, and the radial integration finally leads to the well-known result[26]:

$$
G_{\sigma \sigma}\left(\vec{r}, \vec{r}^{\prime}, E\right)=-\frac{m}{\pi \hbar^{2}} K_{0}\left[-i \frac{\sqrt{2 m}}{\hbar}\left|\vec{r}-\vec{r}^{\prime}\right| \alpha(E \pm \Delta)\right]
$$

where $+(-)$ is for $\sigma=\uparrow(\downarrow), K_{0}$ is the modified Bessel function of the second kind, and

$$
\alpha(x)= \begin{cases}\sqrt{x} & \text { if } x>0 \\ i \sqrt{|x|} & \text { if } x<0\end{cases}
$$

For the perturbation of the $\vec{k} \sigma$ state, as before, we put $E=\varepsilon_{\vec{k} \sigma}$ and express Eq. (B.22) as a function of the momentum $k$. The result is:

$$
G\left(\vec{R}, 0, \varepsilon_{\vec{k} \uparrow}\right)=\beta^{\prime}\left(\begin{array}{cc}
K_{0}(-i k R) & 0 \\
0 & K_{0}\left(-i\left(k^{2}-2 \delta^{2}\right)^{1 / 2} R\right)
\end{array}\right)
$$


where $\delta=(2 m \Delta)^{1 / 2} \hbar^{-1}$ and $\beta^{\prime}=-m /\left(\pi \hbar^{2}\right)$. The same result for the down spin is:

$$
G\left(\vec{R}, 0, \varepsilon_{\vec{k} \downarrow}\right)=\beta^{\prime}\left(\begin{array}{cc}
K_{0}\left(-i\left(k^{2}+2 \delta^{2}\right)^{1 / 2} R\right) & 0 \\
0 & K_{0}(-i k R)
\end{array}\right) .
$$

Writing this in terms of the Pauli matrices as in Eq. (B.11), we get: $g_{0}=$ $\beta^{\prime} K_{0}(-i k R), u=\beta^{\prime} K_{0}\left(\sqrt{2 \delta^{2}-k^{2}} R\right)-g_{0}$, if $-\Delta<\varepsilon_{\vec{k} \uparrow}<\Delta$, and $\beta^{\prime} K_{0}\left(-i \sqrt{k^{2}-2 \delta^{2}} R\right)-$ $g_{0}$, if $\varepsilon_{\vec{k} \uparrow}>\Delta$, and $d=\beta^{\prime} K_{0}\left(-i \sqrt{k^{2}+2 \delta^{2}} R\right)-g_{0}$, if $\varepsilon_{\vec{k} \downarrow}>\Delta$. The remaining algebra is the same as the $3 \mathrm{D}$ case and the final results for the $2 \mathrm{D}$ electron gas reads:

$$
\begin{aligned}
E_{0}^{2 D} & =\frac{-\hbar \lambda}{8 \pi R^{2}}\left(S_{1 z}+S_{2 z}\right)\left(x_{\uparrow}^{2}-x_{\downarrow}^{2}\right), \\
J^{2 D} & =\frac{m \lambda^{2}}{16 \pi R^{2}} \times\left(I_{2}\left(x_{\downarrow}\right)+I_{2}\left(x_{\uparrow}\right)\right), \\
\vec{D}^{2 D} & =0,
\end{aligned}
$$

$$
\begin{aligned}
P^{2 D} & =\frac{m \lambda^{2}}{8 \pi R^{2}} \times\left(-\frac{2}{\pi} \int_{0}^{\eta} \operatorname{Re}\left[K_{0}\left(\sqrt{\eta^{2}-x^{2}}\right)\right]+\int_{\eta}^{x_{\uparrow}} Y_{0}\left(\sqrt{x^{2}-\eta^{2}}\right)\right. \\
& \left.+\int_{0}^{x_{\downarrow}} Y_{0}\left(\sqrt{x^{2}+\eta^{2}}\right)\right) \times x J_{0}(x) d x-J^{2 D} .
\end{aligned}
$$

where, again, $\eta^{2}=2 \delta^{2} R^{2}$, and $I_{2}(x)=x^{2}\left[J_{0}(x) Y_{0}(x)+J_{1}(x) Y_{1}(x)\right]$, the $J$ 's and $Y$ 's here being the Bessel's and Neumann's functions, respectively. In the large distance limit, $I_{2}(x) \rightarrow-\pi^{-1} \sin (2 x)$, so that the magnetic interactions fall off as $\sim R^{-2}$, with a beating pattern similar to the $3 \mathrm{D}$ case, as might be seen from Eq. (B.28) for $J^{2 D}$. Once again, in the non-spin-polarized limit, $k_{F \uparrow}=k_{F \downarrow}$, we recover the standard results for the RKKY interaction for the $2 \mathrm{D}$ electron gas. We note that the spin polarization 
of the substrate was found to be an important ingredient in the spin-spin interaction between the local spins adsorbed on a two-dimensional surface.[9] 


\section{Bibliography}

[1] M. A. Ruderman and C. Kittel, Phys. Rev. 96, 99 (1954).

[2] T. Kasuya, Prog. Theor. Phys. 16, 45 (1956).

[3] K. Yosida, Phys. Rev. 106, 893 (1957).

[4] A. I. Liechtenstein, M. I. Katsnelson, V. P. Antropov and V. A. Gubanov, J. Magn. and Magn. Mat. 67, 65 (1987).

[5] H. Imamura, P. Bruno, and Y. Utsumi, Phys. Rev. B 69, 121303(R) (2004).

[6] F. Parhizgar, H. Rostami and R. Asgari Phys. Rev. B 87, 125401 (2013).

[7] F. Parhizgar, R. Asgari, S. H. Abedinpour, and M. Zareyan, Phys. Rev. B 87, $125402(2013)$.

[8] S. Chesi and D. Loss, Phys. Rev. B 82, 165303 (2010).

[9] J. Fransson, Phys. Rev. B 82, 180411 (R) (2010).

[10] M. Sherafati and S. Satpathy, Phys. Rev. B 83, 165425 (2011); ibid, 84, 125416 (2011). 
[11] T. Skyrme, Nuclear Physics 31, 556 (1962).

[12] N. Nagaosa and Y. Tokura, Nature Nonotech. 8, 899 (2013).

[13] Y. Li, N. Kanazawa, X. Z. Yu, A. Tsukazaki, M. Kawasaki, M. Ichikawa, X. F. Jin, F. Kagawa, and Y. Tokura, Phys. Rev. Lett. 110, 117202 (2013).

[14] N. Romming et al., Science 341, 636 (2013).

[15] E. I. Rashba, Sov. Phys. Solid State 2, 1109 (1960); Y. A. Bychkov and E. I. Rashba, J. Phys. C 17, 6039 (1984); Y. A. Bychkov and E. I. Rashba, JETP Lett. 39, 78 (1984).

[16] I. Dzyaloshinsky, J. Phys. Chem. Solids 4, 241 (1958).

[17] T. Moriya, Phys. Rev. 120, 91 (1960).

[18] Magnetic dipole-dipole interaction: https://en.wikipedia.org/wiki/ Magnetic_dipole

[19] B. A. Lippmann and J. Schwinger, Phys. Rev. 79, p. 469, 1950.

[20] Fubini, G. (1907), "Sugli integrali multipli", Rom. Acc. L. Rend. (5) 16 (1): 608-614, Zbl 38.0343.02 Reprinted in Fubini, G. (1958), Opere scelte 2, Cremonese, pp. 243-249

[21] Friedman, Harvey (1980), "A Consistent Fubini-Tonelli Theorem for Nonmeasurable Functions", Illinois J. Math. 24 (3): 390-395, MR 573474.

[22] Y. Yafet Phys. Rev. B 36, 3948 (1987).

[23] G. F. Giuliani, G. Vignale and T. Datta, Phys. Rev. B 72, 033411 (2005). 
[24] M. M. Valizadeh, International Journal of Modern Physics B 30 (32), 1650234.

[25] I. S. Gradshteyn and I. M. Ryzhik, Tables of Integrals, Series, and Products (Academic Press, New York, 1980), Sec. 8.511.4; M. Sherafati and S. Satpathy, Phys. Rev. B 84, 125416 (2011).

[26] See, for example, M. T. Béal-Monod, Phys. Rev. B 36, 8835 (1987); V. I. Litvinov and V. K. Dugaev, Phys. Rev. B 58, 3584 (1998).

[27] J. A. Sobota, Tanasković and V. Dobrosavljević, Phys. Rev. B 76, 245106 (2007).

[28] M. M. Valizadeh and S. Satpathy, International Journal of Modern Physics B 29 (30), 1550219.

[29] M. Valizadeh and S. Satpathy, physica status solidi (b) 253 (11), 2245-2251.

[30] C. Kittel, in Solid State Physics, vol. 22, edited by F. Seitz, D. Turnbull, and H. Ehreinreich Academic Press, New York, 1968 see also erratum ibidem.

[31] J. Callaway and C. S. Wang, Phys. Rev. B 16, 2095 (1977).

[32] J. Nitta et al., Phys. Rev. Lett. 78, 1335 (1997).

[33] G. Chen et al., Nature Communications 4, 2671-1 (2013).

[34] X. Luo et al., Scientific Reports - 6:18983 — DOI: 10.1038/srep18983.

[35] L. Meier et al., nature physics VOL 3, (2007); doi:10.1038/nphys675.

[36] S.D. Ganichev et al., Phys. Rev. Lett. 92, 256601 (2004)

[37] Yu et al., Nanoscale Research Letters (2016) 11:477 DOI 10.1186/s11671-016$1671-7$. 
[38] G. Dresselhaus, Phys. Rev. 100, 580 (1955).

[39] P. Lyu, N.-N. Liu, and C. Zhang, Gate-tunable Ruderman-Kittel-Kasuya-Yosida interaction mediated by low-dimensional electrons with Rashba spin-orbit coupling, J. Appl. Phys. 102, 103910 (2007).

[40] D.F. Mross and H. Johannesson, Two-impurity Kondo model with spin-orbit interactions, Phys. Rev. B 80, 155302 (2009)

[41] W.-M. Huang, C.-H. Chang, and H.-H. Lin, Noncollinear exchange coupling in a trilayer magnetic junction and its connection to Fermi surface topology, Phys. Rev. B 73, 241307R (2006).

[42] K.-W. Kim, H.-W. Lee, K.-J. Lee, and M. D. Stiles, Chirality from interfacial spin-orbit coupling effects in magnetic bilayers Phys. Rev. lett. 111, 216601 (2013).

[43] A. Kundu and S. Zhang, Dzyaloshinskii-Moriya interaction mediated by spinpolarized band with Rashba, Phys. Rev. B 92, 094434 (2015).

[44] R. Winkler, Spin-Orbit Effects in Two-Dimensional Electron and Hole Systems (Springer, New York, 2003).

[45] K. V. Shanavas and S. Satpathy, Phys. Rev. Letts. 112, 086802 (2014).

[46] B. Fischer and M. W. Klein, Phys. Rev. B 11, 2025 (1975).

[47] B. Andrei Bernevig, et al., Exact SU(2) Symmetry and Persistent Spin Helix in a Spin-Orbit Coupled System, PRL 97, 236601 (2006). 
[48] K. Di, V. L. Zhang, H. S. Lim, S. C. Ng, and M. H. Kuok, Phys. Rev. Lett. 114, $047201(2015)$.

[49] A. Fert and P. M. Levy, Phys. Rev. Lett. 44, 1538 (1980).

[50] J. B. Staunton, B. L. Gyorffy, J. Poulter, and P. Strange, J. Phys. C: Solid State Phys. 21, 1595 (1988).

[51] C. Kittel, Introduction to Solid State Physics, Seventh Ed. (Wiley, New York, 1996).

[52] See, for example, G. Grosso and G. P. Parravicini, Solid State Physics (Academic Press, New York, 2000).

[53] A. A. El-Barbary, R. H. Telling, C. P. Ewels, M. I. Heggie, and P. R. Briddon, Phys. Rev. B 68, 144107 (2003).

[54] P. O. Lehtinen, A. S. Foster, Y. Ma, A. V. Krasheninnikov, and R. M. Nieminen, Phys. Rev. Lett. 93, 187202 (2004).

[55] Y. Ma, P. O. Lehtinen, A. S. Foster, and R. M. Nieminen, New J. Phys. 6, 68 (2004).

[56] V. M. Pereira, F. Guinea, J. M. B. L. dos Santos, N. M. R. Peres, and A. H. C. Neto, Phys. Rev. Lett. 96, 036801 (2006).

[57] O. V. Yazyev and L. Helm, Phys. Rev. B 75, 125408 (2007).

[58] J. Palacios and F. Yndurain, Phys. Rev. B 85, 245443 (2012). 
[59] M. Casartelli, S. Casolo, G. F. Tantardini, and R. Martinazzo, Phys. Rev. B 88, $195424(2013)$.

[60] B. R. K. Nanda, M. Sherafati, Z S Popovic, and S. Satpathy, New J. Phys. 15, 039501 (2013).

[61] C.-K. Lin, J. Chem. Phys. 119, 27131 (2015).

[62] M. G. Menezes and R. B. Capaz, J. Phys. Condens. Matter. 27, 335302 (2015).

[63] Y. Zhang, S.-Y. Li, H. Huang, W.-T. Li, J.-B. Qiao, W.-X. Wang, L.-J. Yin, K.-K. Bai, W. Duan, and L. He, Phys. Rev. Lett. 117, 166801 (2016).

[64] V. G. Miranda, L. G. G. V. Dias da Silva, and C. H. Lewenkopf, Phys. Rev. B 94, $075114(2016)$.

[65] L. Rodrigo, P. Pou, and R. Prez, Carbon bf 103, 200 (2016).

[66] H. Padmanabhan and B. R. K. Nanda, Phys. Rev. B 93, 165403 (2016). 


\section{VITA}

Mohammad M. Valizadeh was born in Iran. He received his undergraduate degree in the field of Physics from University of Tehran. He attended Physics and Astronomy Department at the University of Missouri in fall of 2012, and started to work under supervision of Prof. Sashi Satpathy. He received his Masters and PhD degrees in Computational Physics in 2015 and 2017, respectively. He will start working as a Computational Physicist in industry after graduation.

Here is a list of papers which this thesis is written based on them:

\section{Publications}

1) Mohammad M. Valizadeh and S. Satpathy, International Journal of Modern Physics B 29 (30), 1550219.

2) Mohammad M. Valizadeh and S. Satpathy, physica status solidi (b) 253 (11), $2245-2251$.

3) Mohammad M. Valizadeh, International Journal of Modern Physics B 30 (32), 1650234 .

4) Mohammad M. Valizadeh and Sashi Satpathy, Magnetic Interction in TwoDimensional Electron Gas with Rashba and Dresselhaus Spin-Orbit-Coupling, in preparation. 